\title{
Generalized Euler index, holonomy saddles, and wall-crossing
}

\author{
Dongwook Ghim, ${ }^{a}$ Chiung $\mathrm{Hwang}^{b}$ and Piljin $\mathbf{Y i}^{a}$ \\ ${ }^{a}$ School of Physics, Korea Institute for Advanced Study, \\ Seoul 02455, Korea \\ ${ }^{b}$ Dipartimento di Fisica, Università di Milano-Bicocca \& INFN - Sezione di Milano-Bicocca, \\ Piazza della Scienza 3, I-20126 Milano, Italy \\ E-mail: dghim@kias.re.kr, chiung.hwang@unimib.it, piljin@kias.re.kr
}

AbStRact: We formulate Witten index problems for theories with two supercharges in a Majorana doublet, as in $d=3 \mathcal{N}=1$ theories and dimensional reduction thereof. Regardless of spacetime dimensions, the wall-crossing occurs generically, in the parameter space of the real superpotential $W$. With scalar multiplets only, the path integral reduces to a Gaussian one in terms of $d W$, with a winding number interpretation, and allows an in-depth study of the wall-crossing. After discussing the connection to well-known mathematical approaches such as the Morse theory, we move on to Abelian gauge theories. Even though the index theorem for the latter is a little more involved, we again reduce it to winding number countings of the neutral part of $d W$. The holonomy saddle plays key roles for both dimensions and also in relating indices across dimensions.

KEYwords: Supersymmetric Gauge Theory, Field Theories in Lower Dimensions, Supersymmetry and Duality

ArXIV EPRINT: 1909.11092 


\section{Contents}

1 Motivation 1

1.1 Theories with two-component Majorana supercharges 4

2 Index and wall-crossing: matter only $\quad 6$

2.1 A single scalar: $\mathcal{I}$ vs. $\Omega \quad 7$

2.2 Multiple scalar prototype: $\mathcal{I}$ vs. $\Omega \quad 9$

$2.3 d=1$ index theorem 11

$\begin{array}{ll}2.4 \text { Alternatives for integral } \Omega=\mathcal{I} & 15\end{array}$

$\begin{array}{lll}2.5 & \text { Wall-crossing } & 18\end{array}$

2.6 Uplifts to $d=2,3 \quad 22$

$3 d=1 \mathcal{N}=2$ abelian gauge theories $\quad 22$

$3.1 \Omega$ for gauge theories and holonomy saddles $\quad 23$

3.2 A prototype: $\mathrm{SO}(2)$ with massive charged matters 26

$\begin{array}{ll}3.3 \text { Gaussian reduction } & 27\end{array}$

3.4 Why is Coulombic wall-crossing absent? 34

$4 d=3 \mathcal{N}=1$ Chern-Simons-Matter $\quad 36$

$4.1 d=3 \mathcal{N}=2$ Chern-Simons-Matter revisited 36

$4.2 d=1$ approach for $d=3$ Chern-Simons-Matter 38

4.3 Index theorem for $\mathrm{SO}(2)$ Chern-Simons-Matter 43

$\begin{array}{lll}4.4 & \text { Examples, wall-crossings, and dualities } & 47\end{array}$

A Heat kernel for a Landau problem $\quad 59$

$\begin{array}{ll}\text { B Pfaffian vs. Jacobian } & 60\end{array}$

\section{Motivation}

Since the appearance of the index theorem [1] and its adaptation in physics [2], such topological characterizations became important tools of the trade for studying supersymmetric theories. The path integral reformulation of the index theorem started with the non-linear sigma model by Alvarez-Gaume [3], hence suitable for geometric problems. Since then, numerous generalizations to gauge theories have appeared in literature, and perhaps the most recent and the most systematic descendent are refs. [4, 5] for $d=2$ and ref. [6] for $d=1$ gauge theories. The former computes the elliptic genera while the latter computes $\chi_{y}$ genus, when the theory flows to a compact nonlinear sigma models, and may be considered a sweeping generalization of the Atiyah-Singer index theorems and Alvarez-Gaume's 
path integral reformulation thereof, to a certain class of gauged dynamics with complex supersymmetries.

These recent computations of topological quantities often rely on the so-called localization $[7,8]$. Although one often attributes the resulting simplification to the presence of a BRST operator constructed out of selected supercharges, the real mechanism behind the simplification can be traced to the massive deformation of the theories, which of course is also loosely connected to the choice of the BRST operator. Various flavor chemical potentials as well as flavor masses are typical such, and also immensely helpful is the $R$-symmetry chemical potential, which combined with angular momentum is capable for preserving some supersymmetry. This reduces the problem to a functional Gaussian integral, leaving behind a finite number of integrals, often parameterizing the Cartan directions. Another batch of the simplification arises from the holomorphicity [9], namely how the supersymmetric partition functions depend on external parameters in holomorphic combinations and also how the final phase of the finite integrals reduces to a contour integral of a meromorphic function.

On the other hand, theories with supersymmetries in a Majorana doublet in $d=3$ sense, are qualitatively different. There is no $R$-symmetry in $d=3 \mathcal{N}=1$, and although the dimensional reduction to $d=1$ creates one $\mathrm{U}(1)_{R}$, one cannot introduce its chemical potential because both supercharges transform under it. We could try to turn on flavor chemical potentials, as one would have done for $d=2 \mathcal{N}=(0,2)$ theories. The flavor chemical potentials do invoke a fixed point theorem à la Lefschetz [10], but, as we will see later in this note, tend to disappear from the final form of the twisted partition functions. No notion of the refinement of the index seems possible with the supersymmetry in Majorana doublet.

For geometric theories, the absence of the refinement can be understood also from how $d=2 \mathcal{N}=(1,1)$ does not impose any constraint on the target holonomy. With $\mathcal{N}=(0,2)$, for example, the target would be complex if the theory flows down to a nonlinear sigma model [11], whereby the cohomology decomposes into a Hodge diamond. No such further decomposition of the cohomology is implied with $\mathcal{N}=(1,1)$, so, for example, the Euler number is the only topological quantity that can be constructed out of the cohomology.

This brings us to the question: while we are all familiar with how the Euler number is defined and computed via cohomology when the target is geometric and compact, what would be its analog for general supersymmetric theories? In other words, what are the general features of the Witten index when the supersymmetry is in a Majorana doublet. The subject is hardly new, as this was one of the first class of problems studied by AlvarezGaume [3] in his path integral reformulation of Atiyah-Singer index theorem [1]. For the Euler index, Witten also expanded the discussion into the Morse theory [12], again mostly in the context of compact non-linear sigma model. In this note, we wish to explore a generalized notion of the Euler index for $d=3 \mathcal{N}=1$ theories, and for dimensional reductions thereof, by expanding these pioneering works.

One reason why we come back to this system is the wall-crossing, which turns out to be quite prevalent, and surprisingly so regardless of the spacetime dimensions. Recall how the wall-crossing happens in the more familiar $d=1 \mathcal{N}=4[6]$ but not in its uplift to 
$d=2 \mathcal{N}=(2,2)[11]$. The $d=1$ refined Witten index may experience a sudden jump as a Fayet-Iliopoulos constant $\zeta$ changes the sign $[6,13,14]$, yet the elliptic genus, its $d=2$ analog, does not. This difference can be understood in many different perspectives, but one such is to realize that the phenomenon arises from gapless Coulombic flat direction. The ground state sector of the Coulombic side is notoriously dimension-dependent, yielding such differences between $d=1$ and $d=2$.

On the other hand, with the current, reduced supersymmetry content, the FI-term becomes a term in the superpotential. The would-be Coulombic direction, which is responsible for the wall-crossing, is also spanned by a scalar multiplet [15]. The familiar wall-crossing phenomena must be now attributed to asymptotics along the matter side; as such the strong dependence on spacetime dimensions is no longer necessary. The wallcrossing occurs in the parameter space of the real superpotential. The simplest example is a theory with a single real scalar field $X$ with a quadratic superpotential $W=m X^{2} / 2$, whose index proves to be

$$
\mathcal{I}=\operatorname{sgn}(m),
$$

and the wall of marginal stability occurs at $m=0$. This already hints at how rampant the wall-crossing phenomena would be with this reduced supersymmetry.

Although the compact (geometric) theories admit topologically robust Euler numbers, computable by several well-known methods, study of the Witten index for theories that are more familiar to physics, such as the above free massive thoery, is surprisingly sparse. Furthermore, a systematic study of wall-crossing is much needed. In this note, we will resort to the heat kernel expansion [18] and formulate the twisted partition functions and Witten indices, with emphasis on $d=1$ and on $d=3$. For the latter we restrict our attention to massive theories with discrete classical vacua along the matter side, since for theories with nontrivial classical moduli space of vacua the $d=3$ Witten index itself would be ill-defined. While the latter might be sometimes circumvented by introducing flavor chemical potentials, we will not pursue this here as this in principle represents a heavy-handed deformation of the dynamics [19].

When the theory has scalar multiplets only and a superpotential that admits discrete vacua only, the $d=3$ Witten index is easily computed by $d=1$ Witten index of the dimensionally reduced theory, as the former can be reduced to $d=1$ by putting theory on spatial $\mathbb{T}^{2}$. These cases will be discussed in section 2 , where we reduce the relevant path integral down to a set of ordinary Gaussian integrals. The result connects the Witten index, or a generalized Euler index, to various topological interpretations such as the winding number associated with the superpotential, the Morse theory, and the Lefschetz fixed point theorem. Our main concern in section 2, however, will be the wall-crossings and also finding a formulation that will generalize naturally to the gauged dynamics in the subsequent sections.

With the gauge sector present, vacuum physics is more sensitive to the dimensions. Section 3 deals with $d=1 \mathrm{SO}(2)$ theories, where we again reduce the path integral to a purely Gaussian form, very similar to the matter-only cases in section 2, although the indices, or the twisted partition functions, have even simpler form than the matter only 
cases. The gauge sector often offers additional asymptotic flat directions, which can in principle cause a new type of wall-crossing, but we show that in fact this does not happen. All wall-crossings arise entirely due to the asymptotics of the $d=1 \mathcal{N}=2$ matter sectors, instead. This unexpected behavior can be explained by the mere logical possibility of uplifting the theory to a $d=3$ Chern-Simons theory, as we will discuss at the end of section 3 .

Whenever one considers a supersymmetric partition function of a gauge theory on a circle, the holonomy saddles [20,21] enter the story. For $\mathrm{SO}(2)$ theories, this concept is related to how a gauge-symmetry breaking by charge $q>1$ leaves behind the $\mathbb{Z}_{q}$ discrete subgroup, and how the vacua would be labeled by the discrete holonomies valued in such $\mathbb{Z}_{q}$. In the Hamiltonian view, the relevant holonomies would be those associated with spatial circles in $\mathbb{T}^{d-1}$. In path integrals, however, the $\mathbb{Z}_{q}$ holonomies along the Euclidean time circle also enter, which multiply the above Gaussian integrals by a factor $|q|^{d}$ for theories put on $\mathbb{T}^{d}$. Throughout sections 3 and 4 , this factor will play a crucial role.

In section 4, we move on to computation of Witten indices of $d=3 \mathcal{N}=1 \mathrm{SO}(2)$ Chern-Simons-Matter theories. Note that we should expect immediate and qualitative differences between $d=3 \mathcal{N}=1$ and the better studied $d=3 \mathcal{N}=2[23,24]$. For example, we will see proliferation of wall-crossing in $d=3 \mathcal{N}=1$, while it is well known that the wall-crossing is absent for the latter [24]. As we emphasized earlier, this can be easily understood from its $d=1$ cousins and how the wall-crossing enters the story via the (real) superpotential.

In this last section, we will again manage to reduce the $d=3$ path integral such that the entire Witten index is expressed again in terms of certain winding numbers associated with the superpotential $W$. In particular, much of these winding numbers can be attributed to those of neutral matter multiplets on par with the results in section 2. This in particular means that if the gauge theory has no neutral scalar multiplet, the index takes a rather universal form. We formulate an index theorem complete with a computational routine and close with several examples, as well as a simple check of proposed $d=3 \mathcal{N}=1$ dualities.

We close this summary with a comment on the notation. The main purpose of this note is to reduce path integrals to ordinary Gaussian integrals in the end. This involves a scaling limit of the Euclidean time span $\beta \rightarrow 0$, and an accompanying rescaling of bosonic fields and the superpotentials. We will need to talk about the original field variables, the zero modes, and also $\beta$-rescaled version of the latter. The first two would be denoted by capital alphabet, such as $X$, while the last by the lowercase $x$. However, in the later part of the note, we will take such rescalings for granted and be less strict about the distinction between the original zero mode $X$ and the rescaled one $x$. As such, $X$ 's would be used in place of $x$ 's in many of the middle steps. We made sure to retain $x$ for the final integral formulae, however, as a reminder of $\beta$-rescaling performed to get there.

\subsection{Theories with two-component Majorana supercharges}

We consider a non-chiral supersymmetry, responsible for $d=3 \mathcal{N}=1$ theories or for $d=2 \mathcal{N}=(1,1)$ theories [15]. As for $d=1$, there is a unique superalgebra with two real supercharges, yet one can construct different types of supersymmetric theories [16]. Two 
notable examples have well-recognizable geometric interpretations, sometimes referred to as $\mathcal{N}=2 a$ and $\mathcal{N}=2 b[17]:$ these are related to dimensional reductions of $d=2 \mathcal{N}=(1,1)$ and $d=2 \mathcal{N}=(0,2)$, respectively, and, as such, constructed from very different forms of supermultiplets. For instance, the scalar multiplet in the former has, on shell, two real fermions per a real boson while its counterpart in the latter has a complex fermion matching a complex boson. We will develop techniques for computing supersymmetric partition function for the former class, to be called $d=1 \mathcal{N}=2$ theories in this note for simplicity, ${ }^{1}$ and build back up to $d=2 \mathcal{N}=(1,1)$ and to $d=3 \mathcal{N}=1$.

The supercharges for the theories we wish to study in this note belong to a Majorana doublet, with

$$
\gamma^{0}=i \sigma_{2}, \quad \gamma^{1}=\sigma_{3}, \quad \gamma^{2}=\sigma_{1} .
$$

For the Euclidean signature with which all computations here will proceed, the fermions are pseudo-real, and a convenient basis is

$$
\gamma^{1}=\sigma_{1}, \quad \gamma^{2}=\sigma_{2}, \quad \gamma^{3}=\sigma_{3} .
$$

We can deduce the supermultiplet content of $\mathcal{N}=1$ from those of the more familiar $\mathcal{N}=2$ supersymmetries by halving the supermultiplet.

The basic scalar supermultiplet is literally a half of the more familiar $d=3 \mathcal{N}=2$ chiral multiplet,

$$
\Phi=X+\theta^{a} \psi_{a}-\theta^{2} F,
$$

where the spinor indices are raised and lowered, respectively, by the left and the right multiplication by $C \equiv i \sigma_{2}$, and we also define

$$
\theta^{2}=-\frac{1}{2} \theta^{a} \theta_{a}
$$

With the supercovariant derivative

$$
D_{a} \equiv \partial_{a}+i \theta^{b} \partial_{a b}
$$

where

$$
\partial_{a b} \equiv \gamma_{a b}^{i} \frac{\partial}{\partial x^{i}},
$$

the scalar multiplet has the following superspace Lagrangian,

$$
\mathcal{L}=\frac{1}{4} \int d^{2} \theta\left(D^{a} \Phi D_{a} \Phi\right)+\int d^{2} \theta W(\Phi),
$$

which produces upon the Grassmanian integral,

$$
\frac{1}{2} \partial_{i} X \partial^{i} X+\frac{1}{2} \psi^{a} i \partial_{a}{ }^{b} \psi_{b}+\frac{1}{2}\left(\partial_{X} W\right)^{2}+\psi^{2} \partial_{X}^{2} W(X),
$$

\footnotetext{
${ }^{1}$ We refer readers to ref. [6] for a thorough treatment of $d=1$ supersymmetric partition functions with the latter "complex" realization.
} 
where $W$ is a real superpotential. With more than one scalar multiplet $\Phi^{I}$, we have

$$
\sum_{\mu}\left(\frac{1}{2} \partial_{i} X^{\mu} \partial^{i} X^{\mu}+\frac{1}{2} \psi_{\mu}^{a} i \partial_{a}{ }^{b} \psi_{b}^{\mu}+\frac{1}{2}\left(\partial_{\mu} W\right)^{2}\right)+\sum_{\mu, \nu} \psi^{\mu} \psi^{\nu} \partial_{\mu} \partial_{\nu} W(X),
$$

where $\partial_{\mu}$ stands for the derivative with respect to a real scalar $X^{\mu}$. For matter multiplets, note that we literally divided an $\mathcal{N}=2$ chiral into two equal parts and end up with real scalar multiplets with Majorana fermions.

For vectors, on the other hand, the $\mathcal{N}=2$ vector is split into an $\mathcal{N}=1$ vector $\left(A_{i}, \lambda\right)$ and an $\mathcal{N}=1$ real scalar multiplet $(\Sigma, \Lambda, D)$. The real auxiliary $D$ field belongs to the latter, so one qualitatively new feature is that the would-be the Fayet-Iliopoulos term is actually part of the real superpotential. This fact is rather significant since the FI constant often plays a central role in determining infrared behavior of supersymmetric gauge theories, and in particular controls wall-crossings for $d=1$ theories. As such, the role of superpotential will prove to be central when we consider the Witten index and wall-crossing for theories with a supercharge in a two-component Majorana spinor.

We refer the readers to [15] for a complete detail of the vector supermultiplet and here merely write down a bare-bone feature of the Lagrangian. In addition to the standard kinetic term for $A$ and $\lambda$, a unit-charged complex scalar multiplet $(\Phi, \Psi, F)$ would couple to an $\mathrm{SO}(2)$ vector as follows, for example,

$$
\begin{aligned}
& \frac{1}{2}\left(\partial_{i} X \partial^{i} X+\psi^{a} i \partial_{a}{ }^{b} \psi_{b}\right) \\
\Rightarrow & -\bar{\Phi}(\partial-i A)^{2} \Phi+i \bar{\Psi}^{a}\left(\partial_{a}{ }^{b}-i A_{a}{ }^{b}\right) \Psi_{b}+i\left(\Psi^{a} \lambda_{a} \bar{\Phi}-\bar{\Psi}^{a} \lambda_{a} \Phi\right),
\end{aligned}
$$

where we now took the matter multiplets to be complex, $\Phi=\left(X^{1}+i X^{2}\right) / \sqrt{2}$, etc. In section 2, Abelian vector multiplets will make a brief appearance as external objects that make the theory massive. In sections 3 and 4 , on the other hand, we will consider gauge theories where the vector multiplet becomes dynamical.

\section{Index and wall-crossing: matter only}

In this section, we will explore the usual heat kernel expansion [18] further, with an aim at handling theories with wall-crossing phenomena or continuum sector or both. We start with $d=1 \mathcal{N}=2$ theories with two real supercharges [3]. The canonical Hamiltonian for scalar multiplets is

$$
\mathcal{H} \equiv \sum_{\mu=1}^{N}\left(\frac{1}{2} \pi_{\mu} \pi_{\mu}+\frac{1}{2}\left(\partial_{\mu} W\right)^{2}\right)+\sum_{\mu, \nu}^{N} \psi^{\mu} \psi^{\nu} \partial_{\mu} \partial_{\nu} W(X),
$$

with the canonical conjugate momenta $\pi_{\mu}$ to $X^{\mu}$ and the two sets of Grassman variables each spanning a Clifford algebra,

$$
\left\{\psi_{a}^{\mu}, \psi_{b}^{\nu}\right\}=\delta^{\mu \nu} \delta_{a b}
$$

The quantity we wish to compute is the twisted partition function,

$$
\operatorname{Tr}(-1)^{\mathcal{F}} e^{-\beta \mathcal{H}}
$$


with two interesting limits,

$$
\begin{aligned}
& \mathcal{I}=\lim _{\beta \rightarrow+\infty} \operatorname{Tr}(-1)^{\mathcal{F}} e^{-\beta \mathcal{H}}, \\
& \Omega=\lim _{\beta \rightarrow 0^{+}} \operatorname{Tr}(-1)^{\mathcal{F}} e^{-\beta \mathcal{H}} .
\end{aligned}
$$

The former is the desired Witten index [2], while the latter, so-called bulk part, is the one that can be compute systematically. ${ }^{2}$

The trace over the Hilbert space reduces to the following integral,

$$
\operatorname{Tr}(-1)^{\mathcal{F}} e^{-\beta \mathcal{H}}=\int d^{N} X \operatorname{tr}_{\psi}\left((-1)^{\mathcal{F}}\left\langle X\left|e^{-\beta \mathcal{H}}\right| X\right\rangle\right),
$$

where the $\operatorname{tr}_{\psi}$ means the trace over the spinor representation of the algebra (2.2), where $(-1)^{\mathcal{F}}$ is nothing but the usual chirality operator. The small $\beta$ limit of this expression computes the so-called bulk index $\Omega$, or the twisted partition function. The small $\beta$ expansion proceeds as

$$
\Omega \equiv \lim _{\beta \rightarrow 0^{+}} \operatorname{Tr}(-1)^{\mathcal{F}} e^{-\beta \mathcal{H}}=\lim _{\beta \rightarrow 0^{+}} \frac{\beta^{N}}{(2 \pi \beta)^{N / 2}} \int d^{N} X \operatorname{det}\left(\partial_{\mu} \partial_{\nu} W\right) e^{-\beta(\partial W)^{2} / 2},
$$

where we used the standard Heat kernel expansion with the familiar zero-th order part,

$$
\left\langle\tilde{X}\left|e^{\beta \partial_{X}^{2} / 2}\right| X\right\rangle=\frac{1}{(2 \pi \beta)^{N / 2}} e^{-(\tilde{X}-X)^{2} / 2 \beta},
$$

in the $N$ dimensional Euclidean space $\mathbb{R}^{N}$. With sufficiently gapped $W$, on the other hand, (2.6) should in turn equal the integral index $\mathcal{I}$.

Along the way to (2.6), it is instructive to note that the determinant prefactor is actually a Pfaffian,

$$
\operatorname{det}\left(\partial_{I} \partial_{J} W\right) \Leftarrow \operatorname{Pf}\left(\epsilon_{a b} \partial_{\mu} \partial_{\nu} W\right)
$$

since the fermion bilinear responsible for this contribution has the form

$$
\epsilon_{a b} \psi^{\mu a} \psi^{\nu b} \partial_{\mu} \partial_{\nu} W
$$

with Majorana $\psi$ 's. When we introduce the gauge symmetry, it is the latter that will be generalized straightforwardly.

\subsection{A single scalar: $\mathcal{I}$ vs. $\Omega$}

For the simplest illustration, consider a single scalar multiplet with a polynomial $W$ with the leading power $k, W=c_{k} X^{k} / k !+\cdots$. Rescaling $X$ by $x \equiv \beta^{1 / 2(k-1)} X$, we remove the

\footnotetext{
${ }^{2}$ As has been emphasized elsewhere [19], the so-called localization method for supersymmetric partition functions in the presence of a circle often computes the analog of $\Omega$, even though there it may appear that no particular limit of $\beta$ is taken. Since $\beta$ is dimensionful, it is really a dimensionless combination of $\beta$ with some other scaling parameter that is implicitly taken to zero in such a localized path integral. Insertion of the chemical potential can retain some memory of finite circle size [21], yet the parameter sitting in front of $H$ or more precisely $\mathcal{Q}^{2}$ is effectively taken to zero at the end of the localization process.
} 
power of $\beta$ in the prefactor; this also allows us to ignore lower powers of $X$ in $W$. Then we end up with

$$
\Omega=\frac{1}{(2 \pi)^{1 / 2}} \int_{-\infty}^{\infty} d x \frac{c_{k} x^{k-2}}{(k-2) !} e^{-\left(c_{k} x^{k-1} /(k-1) !\right)^{2} / 2} .
$$

For odd $k$, this is an integral of odd function, hence we find

$$
\mathcal{I}=0 .
$$

With $k$ even, the integral reduces to a Gaussian integral we will encounter repeatedly below,

$$
\frac{1}{(2 \pi)^{1 / 2}} \int_{-\infty \cdot \operatorname{sgn}\left(c_{k}\right)}^{\infty \cdot \operatorname{sgn}\left(c_{k}\right)} d y e^{-y^{2} / 2}
$$

with $y \equiv c_{k} x^{k-1} /(k-1)$ !, so that

$$
\mathcal{I}=\Omega=\operatorname{sgn}\left(c_{k}\right) .
$$

Another way to understand these results is to recall that the supersymmetric wavefunction in this simple theory is proportional to

$$
\Psi_{ \pm}(X) \sim e^{ \pm W(X)},
$$

where the sign in the exponent is correlated with the chirality $(-1)^{\mathcal{F}}$ of the wavefunction. Clearly $W(X)$ must diverge at the two asymptotic infinities $X \rightarrow \pm \infty$ with a common same sign, for one of these two states to be physically acceptable, so $k$ should be even for a ground state to exist. This also tells us the above result goes well beyond the polynomial form of the superpotential we assumed for the sake of convenience.

It is instructive to redo this computation with slightly different scaling, which sheds more light. Suppose we chose to scale coefficients of the lower powers of $X$ such that $a_{k-\delta} \equiv \beta^{\delta / 2(k-1)} c_{k-\delta}$ kept finite,

$$
\left.\widetilde{W}(x) \equiv W(X)\right|_{X \rightarrow x ; c_{n} \rightarrow a_{n}}
$$

so that the integrand is

$$
\int d x \widetilde{W}^{\prime \prime}(x) e^{-\left(\widetilde{W}^{\prime}(x)\right)^{2} / 2}=d y e^{-y^{2} / 2}
$$

with $y \equiv \widetilde{W}^{\prime}(x)$. Note how all the information is now transferred to the integral range via the map $x \rightarrow \widetilde{W}^{\prime}(x)$. In fact this can be done for any real function $W$, so we may as well consider $\widetilde{W}(x)$ an arbitrary smooth function.

Since one can generally find places where $\widetilde{W}^{\prime \prime}(x)=0$, the integral range of $y$ is in general composed of multiple overlapping segments. Suppose the range of $y$ integration decomposes into $\left[y_{L} \equiv \widetilde{W}^{\prime}(-\infty), y_{1}\right] \cup\left[y_{1}, y_{2}\right] \cup \cdots \cup\left[y_{k-2}, y_{R} \equiv \widetilde{W}^{\prime}(\infty)\right]$. The adjacent segments will cancel, at least partially, and the net integration range is $\left[y_{L}, y_{R}\right]$,

$$
\Omega=\frac{1}{(2 \pi)^{1 / 2}} \int_{y_{L}}^{y_{R}} d y e^{-y^{2} / 2} .
$$


This non-integral result can be understood from the fact that $y_{L, R}$ control the asymptotic flat directions along $X= \pm \infty$. If these numbers are finite, it means that we can have a continuum sector contribution to $\Omega$, only to be suppressed by $e^{-y_{L, R}^{2} / 2}$.

In order to count the genuine ground states, unaffected by the finite values of these asymptotic potentials, one may scale up the entire potential $W$ by an arbitrary large positive number, say $C$. As long as neither of $y_{L, R}$ is zero, the problem is Fredholm, and the true index would be robust under such a deformation. Then,

$$
\Omega_{C} \equiv \frac{1}{(2 \pi)^{1 / 2}} \int_{y_{L} \times C}^{y_{R} \times C} d y e^{-y^{2} / 2}
$$

produces

$$
\mathcal{I}=\lim _{C \rightarrow \infty} \Omega_{C}=\left\{\begin{aligned}
1 & y_{L}<0<y_{R} \\
0 & y_{L} \times y_{R}>0 \\
-1 & y_{L}>0>y_{R}
\end{aligned}\right.
$$

which of course reduces to the standard result, in accord with the explicit wavefunction construction (2.14).

From this simple exercise we learn a few valuable things about $\Omega$. First of all, if the asymptotic potential approaches a finite value, $\Omega$ won't be in general integral but will be contaminated by continuum, exponentially suppressed by $e^{-\beta W^{\prime}( \pm \infty)^{2} / 2}$. It is important that we keep $\beta$ small but finite, despite the usual heat kernel expansion, since, otherwise, we would lose control over the continuum contributions. Since the index is robust as long as the asymptotic dynamics is qualitatively unaltered, we should take care to keep the functional form of the combination $\beta\left(W^{\prime}\right)^{2}$ intact and finite in the middle steps, even as $\beta \rightarrow 0$. As long as the problem is Fredholm, we always have a choice to scale up $W$ in the end without affecting the true ground state sector, producing the true index $\mathcal{I}$ at the very end of the process.

Note that, already for this simplest of theories, the system can undergo wall-crossing at a codimension-one wall where one of the two asymptotic values $W^{\prime}( \pm \infty)$ crosses zero. In order to catch this, it is advantageous to postpone the scaling (2.19) such that $\Omega$ remains a smooth function of coupling constants: again, we must not be hasty in deforming away details of the superpotential, even if naive topological robustness seems to allow it. A simple corollary of $(2.19)$ is,

$$
\mathcal{I}_{W}-\mathcal{I}_{-W}= \pm 2
$$

where $\mathcal{I}_{W}$ is the index for the theory of a single scalar multiplet with the superpotential $W$. This gives the prototype wall-crossing behavior of all. The superpotential $W$, or more precisely its asymptotic leading part crosses a co-dimoension-one wall, and this forces the generalized Euler index will jump by \pm 2 somewhere in the middle.

\subsection{Multiple scalar prototype: $\mathcal{I}$ vs. $\Omega$}

With more-than-one scalar multiplets, the quantity that controls the continuum contributions and the potential wall-crossing behavior is the smallest possible asymptotic value of 
$\left(\partial_{\mu} W\right)^{2}$. We will again concentrate on a prototype where this quantity is finite, and see what should be done to deal with the continuum sector. Take $n X_{i}$ 's, collectively denoted by $\mu$-component vector $\vec{X}$, and a single $P$ such that

$$
W(P ; \vec{X})=\frac{1}{2} P\left(\vec{X}^{2}-\xi\right),
$$

which leads to

$$
\partial_{P} W=\frac{\left(\vec{X}^{2}-\xi\right)}{2}, \quad \partial_{\mu} W=P X_{\mu}
$$

and

$$
\partial_{P} \partial_{P} W=0, \quad \partial_{P} \partial_{\mu} W=X_{\mu}, \quad \partial_{\mu} \partial_{\nu} W=P \delta_{\mu \nu} .
$$

The integral becomes, after rescaling the $n+1$ fields upon which $\xi$ is effectively scaled down to 0 , by $\beta^{1 / 4}$,

$$
\Omega=\frac{1}{(2 \pi)^{(n+1) / 2}} \int d p d^{n} x p^{n-1} \vec{x}^{2} e^{-\left(p^{2} \vec{x}^{2}+\left(\vec{x}^{2} / 2\right)^{2}\right) / 2},
$$

which already shows $\mathcal{I}=0$ for even $n$. For odd $n$, we have

$$
\Omega=\frac{V_{n-1} \Gamma(n / 2)}{\pi^{(n+1) / 2} \sqrt{2}} \int_{0}^{\infty} d\left(r^{2} / 2\right) e^{-\left(\left(r^{2} / 2\right)^{2}\right) / 2}=1,
$$

where we integrated out $p$ and the angular part of $\vec{x}$ first, which led to the angular volume $V_{n-1}$ of a unit $(n-1)$-sphere.

Note that, however, the anticipated index $\mathcal{I}$ differs from this $\Omega$. If $\xi>0$, the vacuum manifold is an $(n-1)$-dimensional sphere, $\hat{\mathbb{S}}^{n-1}$ defined by $\vec{X}^{2}=\xi$ and $P=0$, with the Euler index equal to 2 . If $\xi<0$, on the other hand, the vacuum manifold is absent entirely, so the index should be zero. The above result, $\Omega=1$, even though it is suggestively integral, does not really count the true ground states for any value of $\xi$.

Again this misleading answer appears because the path integral can admit contributions from continuum sectors. In this prototype, even though the asymptotic potential grows quadratically for generic direction, there is a valley of $\vec{X}=0$, where the bosonic potential equals $V(P ; \vec{X}=0)=\xi^{2} / 8$ for any value of $P$. The twisted partition function can be contaminated by continuum, suppressed as

$$
\sim e^{-\beta \xi^{2} / 8}
$$

The suppression factor happens to 1 as $\beta \rightarrow 0$, resulting in an integral $\Omega$, which does not equal the desired index $\mathcal{I}$. The continuum sector conspires to contribute to $\Omega$ a nonzero integer value on top of $\mathcal{I}$, potentially causing much confusions. The above small $\beta$ computation, which would have been acceptable in more ideal circumstances with fully discrete Hilbert space, cannot be trusted to distinguish the continuum contribution from the true index. 
Therefore, scaling away details of $W$ is not wise; we should have kept the combination $\beta \xi^{2}$ finite until the very end of the computation. Instead of (2.24), therefore, we are lead to consider

$$
\Omega=\frac{V_{n-1} \Gamma(n / 2)}{\pi^{(n+1) / 2} \sqrt{2}} \int_{0}^{\infty} d\left(r^{2} / 2\right) e^{-\left(\left(r^{2} / 2-\sqrt{\beta} \xi / 2\right)^{2}\right) / 2} .
$$

The deviation of $\Omega$ from these integral values are of order $\sim e^{-\beta \xi^{2} / 8}$ as expected. As long as $\xi \neq 0$, the problem is Fredholm and we are allowed to take the $\beta \xi^{2} \rightarrow \infty$ limit while preserving the true ground state contributions, from which we find

$$
\mathcal{I}=\lim _{\beta \xi^{2} \rightarrow \infty} \Omega=\left\{\begin{array}{ll}
2 & \xi>0 \\
0 & \xi<0
\end{array},\right.
$$

and the correct integral index emerges at the end of the process.

This behavior is reminiscent of an early confusion and the subsequent resolution [6] with the localization for gauged quantum mechanics, with complex supersymmetries. The localization naively claimed that the index is inert under the continuous change of the FI constant $\xi$, which is a BRST trivial deformation, yet this was clearly at odd with the well-known wall-crossing phenomena. As here, the resolution came from the asymptotic flat direction with gap $\sim \xi^{2}$, which induces wall-crossing at $\xi=0$. With half and nonchiral supersymmetry we are considering, the would-be FI constants are now part of the superpotential for real scalar multiplets, and yet still lead to wall-crossing. This means that the wall-crossing now occurs quite generically for continuous deformations of the superpotential. The question is whether there is a similar universal scheme for computing the integral index for non-chiral two-supercharge problems at hand.

\section{$2.3 d=1$ index theorem}

What we have seen so far tells us to keep the functional form of $W$ intact in the $\beta \rightarrow 0$ limit. One easy way to achieve this is to redefine the bosonic zero modes somewhat differently than the above,

$$
\beta^{-1 / 2} X \quad \rightarrow \quad x
$$

after the integration over the fermions. Note that this is a dimensionless combination in $d=1$. Another advantage of this choice is that $W$ need not be restricted to a polynomial form; any real and twice-differentiable $W$ will do. This transforms (2.6) into

$$
\Omega=\frac{1}{(2 \pi)^{N / 2}} \int d^{N} x \operatorname{det}\left(\partial_{\mu} \partial_{\nu} w(x)\right) e^{-\left(\partial_{\mu} w(x)\right)^{2} / 2},
$$

with replacement

$$
w(x)=\left.W(X)\right|_{X \rightarrow x}
$$

Note that, in view of (2.29), this last replacement is possible only with some complicated rescalings of parameters that go into the definition of $W$, much as in the previous subsection but now including an overall multiplication. 
One may view this a new definition of $\Omega$. Of course, the point is that any such rescaling is harmless as long as computation of $\mathcal{I}$ goes. This exponent is still proportional to $\beta$, hidden in $\partial_{\mu}$, but the point is that we will take $\partial_{\mu} w$ as the new integration variables and transfer all information, including the $\beta$ dependence, to the integration range information. In the end, the only relevant information, as far as the final index $\mathcal{I}$ goes, would be whether or not the integration range includes the origin $\partial w=0$ with what multiplicity.

To be more explicit, one introduces new variables $y_{\mu}=\partial_{\mu} w(x)$ and finds

$$
\begin{aligned}
& \Omega=\Omega_{Y} \equiv \int_{Y} \mu, \\
& \mu=\frac{1}{(2 \pi)^{N / 2}}\left(e^{-y_{1}^{2} / 2} d y_{1}\right) \wedge\left(e^{-y_{2}^{2} / 2} d y_{2}\right) \wedge \cdots \wedge\left(e^{-y_{N}^{2} / 2} d y_{N}\right),
\end{aligned}
$$

where all the nontrivial information is now transferred into the integration domain

$$
Y=\cup_{\Delta} Y_{\Delta}, \quad Y_{\Delta} \equiv\left\{\partial_{\mu} w(x) \mid x_{\mu} \in \Delta \subset \mathbb{R}^{N}\right\}
$$

where each $\Delta$ is defined so that $\mathbb{R}^{N}=\cup \Delta$ and each $\Delta$ is a maximal domain in which the map $x_{\mu} \rightarrow \partial_{\mu} w(x)$ induces a unique orientation in the target $Y_{\Delta} \subset \tilde{\mathbb{R}}^{N}$ from that of $\mathbb{R}^{N}$. The integral over $Y_{\Delta}$ will naturally encode this orientation via a \pm sign.

The general ideas behind the index, and our treatment of the path integral so far, say the following: (1) If the asymptotic flat directions are absent altogether, we will find $\Omega$ count the number of ground states correctly and

$$
\mathcal{I}=\Omega_{Y}
$$

by itself; (2) If there exists an asymptotic flat direction with a positive energy gap, one will find deviation from $\mathcal{I}$ by an exponentially suppressed term, which can be removed by a further scaling $w(x) \rightarrow C \cdot w(x)$ by an arbitrarily large positive number $C$

$$
\mathcal{I}=\lim _{C \rightarrow+\infty} \Omega_{C Y}
$$

where $C Y$ is the scale-up version of $Y$ by the factor $C$; (3) Finally, if there is an asymptotic flat direction with no energy gap, $\Omega$ can be generally non-integral and such continuum contributions cannot be removed within this straightforward path integral setup by itself; more refined approaches need to be invoked.

The (non-)integrality. To show that these assertions indeed hold with (2.32), we start by noting that each Gaussian integral produces $\pm \sqrt{2 \pi} / \sqrt{2 \pi}= \pm 1$ if the range is over the entire real line. Therefore, the integral there would be an integer if each $Y_{\Delta}$ equals $\tilde{\mathbb{R}}^{N}$, up to the orientation. But of course the life is not that simple.

The claim that (2.35) will give an integer amounts to the assertion that,

$$
C Y=\cup_{\Delta} C Y_{\Delta}, \quad C Y_{\Delta} \equiv\left\{C \cdot \partial_{\mu} w(x) \mid x^{\mu} \in \Delta \subset \mathbb{R}^{N}\right\}
$$

asymptotes to a multiple cover of $\tilde{\mathbb{R}}^{N}$, after the orientation is taken into account, as $C \rightarrow$ $+\infty$. This will happen, clearly, if and only if all asymptotic flat directions, if any, come 
with nonzero energy gap. It is clear that a gapped asymptotic flat direction will create boundaries in some of $Y_{\Delta}$, which however do not intersect the origin. As such, the scaled version, $C Y_{\Delta}$, would push out such boundaries by the factor $C$. If the origin is contained in any open subset of $Y_{\Delta}, C Y_{\Delta}$ would expand and fill the entire $\tilde{\mathbb{R}}^{N}$, so that

$$
\lim _{C \rightarrow \infty} \int_{C Y_{\Delta}} \mu=\int_{ \pm \tilde{\mathbb{R}}^{N}} \mu= \pm 1
$$

If the origin lies outside of $Y_{\Delta}, C Y_{\Delta}$ would be pushed out to the asymptotic region, so that

$$
\lim _{C \rightarrow \infty} \int_{C Y_{\Delta}} \mu=0
$$

This leads to

$$
\lim _{C \rightarrow \infty} \int_{C Y} \mu=\lim _{C \rightarrow \infty} \sum_{\Delta} \int_{C Y_{\Delta}} \mu \in \mathbb{Z}
$$

which should equal the index $\mathcal{I}$. This confirms (2) above, and thus the index formula (2.35).

Boundaries of individual $Y_{\Delta}$ could come either from the asymptotic behavior of $w(x)$ or due to a linear combination of $\partial_{\mu} w(x)$ bouncing at finite values of $x$, where one necessarily expect $0=\operatorname{det}(\partial \partial w)$. So far, we overlooked the latter boundaries but as long as such a boundary does not meet the origin, i.e., $0=(\partial w)^{2}$, the scaling by $C$ will remove it as well. What happens if such a boundary happens to meet the origin of $\tilde{\mathbb{R}}^{N}$ ? One easy way to see how boundaries of $Y_{\Delta}$ 's at finite $x$ are irrelevant for the ground state counting is the following: since we are really after the integer quantity $\mathcal{I}$, rather than $\Omega$, and since the former is robust under a "small" deformation of $W(X)$, one can easily deform $w(x)$ such that the troublesome boundary is shifted slightly away from the origin, $y=0$, while maintaining the boundaries due to $\vec{x}^{2} \rightarrow \infty$ intact. This will of course affect both of $Y_{\Delta}$ 's in question but in such a manner that the integral part $\mathcal{I}$ is unaffected. Afterward we are free to scale the deformed $w$ by $C$ and obtain $\mathcal{I}$ via (2.35).

By the same token, we see how the integrality can fail for (3). If there is a gapless asymptotic direction, this means

$$
(\vec{\partial} w)^{2} \rightarrow 0
$$

along some asymptotic direction, $\vec{x}^{2} \rightarrow \infty$. This allows a boundary of some $Y_{\Delta}$ to meet the origin, which can, generically, neither fill $\tilde{\mathbb{R}}^{N}$ nor be pushed out to infinity by a simple overall scaling by $C$. Invoking a perturbation of $W$ at such a place can lift the problematic direction in principle but produces multiple different outcomes for $\mathcal{I}$. In fact, the wall-crossing happens precisely because different deformations that lift such gapless flat directions generally lead to different integral indices.

Finally we need to show the integrality of $\Omega$ itself for the case (1), where there is no asymptotic flat direction at all. This condition implies

$$
(\vec{\partial} w)^{2} \rightarrow \infty \quad \text { as } \quad \vec{x}^{2} \rightarrow \infty
$$


for all possible directions $\vec{x}$. The integrality would follow immediately if $Y$, after possible cancelation between $Y_{\Delta}$ 's due to mutually opposite orientations, is itself a multiple-cover of $\tilde{\mathbb{R}}^{N}$. Since a boundary of each component can come only from finite $x$, there should be necessarily a pair, say $Y_{\Delta_{1}}$ and $Y_{\Delta_{2}}$ meeting at such a boundary. These pairs, by definition, overlap in $\tilde{\mathbb{R}}^{N}$ with mutually opposite orientations. The two can then be combined to a third domain $Y_{\Delta_{3}}$ in $\tilde{\mathbb{R}}^{N}$. If the latter has a boundary from finite $x$, we then find another adjacent and canceling domain $Y_{\Delta_{4}}$, and so on. In the end, all boundaries originating from finite $x$ will effectively disappear. Since those from the asymptotic $x, \vec{x}^{2} \rightarrow \infty$ are absent to begin with, $Y$ has to be a multiple cover $\tilde{\mathbb{R}}^{N}$ and $\Omega$ has to be integral and thus equal to $\mathcal{I}$, resulting in (2.34).

Back to the examples. Let's redo the two examples we started with, using this language. If one starts with $\mathcal{N}=1$ with $k$-th order polynomial $w(x)$, we will generically have $k-1 \Delta$ 's and $Y_{\Delta}$ 's, divided by $0=w^{\prime \prime}(x)$. Ordered by the natural ordering of the real line $x$, each pair of adjacent $Y_{\Delta}$ 's comes with mutually opposite orientations, so cancels out partially. For instance, $w(x)=x^{3} / 3$ gives $y=x^{2}$, with $\mathbb{R}=\Delta_{-} \cup \Delta_{+}$and $\Delta_{ \pm}= \pm(0, \pm \infty)$ where the sign \pm in front denotes the orientation. Mapping to $y$ variables, this gives

$$
Y_{\Delta_{ \pm}}= \pm(0,+\infty)
$$

which cancel each other. Proceeding similarly for arbitrary power $k$, one can show easily that

$$
Y=\operatorname{sgn}\left(c_{k}\right) \tilde{\mathbb{R}} \text { for even } k ; Y=\emptyset \text { for odd } k
$$

with the orientation for the former, fixed by the sign of the coefficient $c_{k}$ of $x^{k}$. Hence $\mathcal{I}=\operatorname{sgn}\left(c_{k}\right)$ for even $k \geq 2$ and 0 otherwise.

For the multi-scalar prototype above, the map

$$
y_{p}=\left(\vec{x}^{2}-\xi\right) / 2, \quad y_{i}=p x_{i}
$$

admits two $\Delta$ 's such that

$$
\begin{array}{ll}
\Delta_{1}=-[0,-\infty)_{p} \times \mathbb{R}_{x}^{n}, & Y_{\Delta_{1}}=-[-\xi / 2,+\infty) \times(-1)^{n} \tilde{\mathbb{R}}^{n}, \\
\Delta_{2}=[0,+\infty)_{p} \times \mathbb{R}_{x}^{n}, & Y_{\Delta_{2}}=[-\xi / 2,+\infty) \times \tilde{\mathbb{R}}^{n} .
\end{array}
$$

For even $n$, the two $Y_{\Delta}$ 's cancel out each other precisely. For odd $n$, the two scaled-up domains,

$$
C Y_{\Delta_{1}}=C Y_{\Delta_{2}}=[-C \xi / 2,+\infty) \times \tilde{\mathbb{R}}^{n} \rightarrow \tilde{\mathbb{R}}^{n+1} \quad \text { as } C \rightarrow+\infty
$$

contribute 1 each for $\xi>0$, again resulting in $\mathcal{I}=2$. For $\xi<0, \mathcal{I}=0$ because the two domains are pushed out to infinity under the $C$ scaling. 


\subsection{Alternatives for integral $\Omega=\mathcal{I}$}

When the twisted partition function $\Omega$ actually equals $\mathcal{I}$ and thus integral, there are several alternative forms of this index (2.32) under additional assumptions, well known in the relevant literatures. In particular, the Morse theory [12] is a direct consequence of (2.32), while the winding number interpretation will later prove to be very useful for polynomial $W$. Both of these alternatives rely on generic form of the superpotential, which could fail if certain global symmetries are imposed. For the latter, the Lefschetz fixed point theorem comes to the rescue as usual, however.

The winding number interpretation. The integration formula (2.32) counts in the end how many times the map $X \rightarrow d W$ covers $\tilde{\mathbb{R}}^{N}$, with the orientation taken into account. Recall that $\Omega=\mathcal{I}$ is guaranteed if $|d W|^{2} \rightarrow \infty$ along all asymptotic directions in the $X$ space. Suppose further that the superpotential and its derivatives are regular everywhere at finite $X$. The continuity of the map then implies that $\Omega=\mathcal{I}$ can be also counted by the winding number of the map,

$$
\left.\vec{\varphi}_{R}(\hat{X}) \equiv \frac{\vec{\partial} W(X)}{|\vec{\partial} W(X)|}\right|_{X=R \hat{X}}
$$

with $|\hat{X}|=1$, from $\mathbb{S}^{N-1}$ to $\hat{\mathbb{S}}^{N-1}$, which is well-defined for sufficiently large $R$. More explicitly, we have

$$
\mathcal{I} \cdot \int_{\hat{\mathbb{S}}^{N-1}} \hat{\mathcal{V}}=\int_{\mathbb{S}^{N-1}} \varphi_{R}^{*} \hat{\mathcal{V}}
$$

at some large $R$, where $\hat{\mathcal{V}}$ is the volume form of $\hat{\mathbb{S}}^{N-1}$ and $\varphi_{R}^{*}$ is the pull-back.

While this is not a very practical form, e.g., for numerical computation, it offers an ultraviolet perspective, opposite of the Morse theory interpretation below, and instructs us that the asymptotic $W$ suffices for the enumerative $\mathcal{I}=\Omega$. This also implies, as we study in the next subsection, that the codimension-one wall-crossing is also controlled entirely by the asymptotic form of $W$.

For instance, suppose $N$ is odd and $W(-X)=-W(X)$. For such cases, (2.30) immediately implies $\mathcal{I}=0$ identically; the Pfaffian contribution flips the sign under $X \rightarrow-X$, so the integral is odd under the parity. Note that the same vanishing theorem manifests in (2.48) in how $\varphi^{*} \mathcal{V}$ contributions cancel between the antipodal points pairwise for odd $N$. On the other hand, (2.48) does not depend on details of $W$ at finite $X$. Only the asymptotic part matters. Therefore, for odd $N$, whenever one can identify an approximate $W^{\text {asymp }}$, such that $W^{\text {asymp }}(-X)=-W^{\text {asymp }}(X)$ and that

$$
\frac{W^{\text {asymp }}-W}{W} \rightarrow 0 \quad \text { as }|X| \rightarrow \infty
$$

along all directions, we may conclude

$$
\mathcal{I}_{W}=0
$$

regardless of details of $W$ at finite $X$ region. 
One obvious example of this phenomenon occurs when the superpotential has a polynomial form. Suppose that the superpotential starts with the leading power $p+1$,

$$
W(X)=W^{(p+1)}(X)+\cdots .
$$

We can say immediately that, with odd $N, \mathcal{I}$ computed from (2.48) is identically zero if $p+1$ is also odd, whenever the homogeneous $W^{(p+1)}(X)$ is nondegenerate. The latter is in turn guaranteed by the genericity of the superpotential in the absence of global symmetries. When a global symmetry exists, on the other hand, we may resort to a Lefschetz fixed point theorem outlined below.

The Morse theory interpretation. Looking at (2.32), or more precisely at its $C$-scaled version (2.35), one realizes that it also counts the critical points of the superpotential, $d W=0$, provided that the latter are all isolated and nondegenerate. As we scale up $W(x)$ by a large multiplicative factor $C$, the integral is localized to its critical points, $d W\left(x_{*}\right)=0$, in the $\vec{x}$ space. The Gaussian integral over a single $\Delta$ domain would converge to $(-1)^{\gamma\left(x_{*}\right)}$ where $\gamma\left(x_{*}\right)$ is the so-called Morse index [12] of $x_{*}$, i.e., the number of negative eigenvalues of the Hessian $\partial_{\mu} \partial_{\nu} W\left(x_{*}\right)$. Each Gaussian integral gives \pm 1 , depending on whether the relevant range is $(-\infty, \infty)$ or $(\infty,-\infty)$, and the combined sign at each $x_{*}$ is dictated by the sign of the determinant of the Hessian.

Naturally, if critical points of $W$ are discrete and nondegenerate and all distributed over a closed subregion of $\tilde{\mathbb{R}}^{N}$, we find

$$
\mathcal{I}=\lim _{C \rightarrow \infty} \Omega_{C Y}=\sum_{x_{*}}(-1)^{\gamma\left(x_{*}\right)} .
$$

Here again we can see that for odd $N, W(X)=W^{\operatorname{asymp}}(X)+\cdots$ with odd $W^{\text {asymp }}(X)$ in the sense of (2.49) leads to $\mathcal{I}_{W}=0$. Consider $W_{1}(X)=-W(X)$ and $W_{2}(X)=W(-X)$. If $x_{*}$ is a critical point of $W$, it is also a critical point of $W_{1}$ and vice versa, and $-x_{*}$ is obviously a critical point of $W_{2}$. The sign of the Hessian for $W_{1}$ at $x_{*}$ and that of $W_{2}$ at $-x_{*}$ have the sign $(-1)^{N}$ and $(-1)^{2 N}$ relative to that of $W$ at $x_{*}$, point by point, which give

$$
\mathcal{I}_{W_{2}}=\mathcal{I}_{W}=-\mathcal{I}_{W_{1}}
$$

for odd $N$. On the other hand, $W_{1}$ and $W_{2}$ share a common leading asymptotic form $W_{1}^{\text {asymp }}(X)=W_{2}^{\text {asymp }}(X)=-W^{\text {asymp }}(X)$, for odd $W^{\text {asymp }}$, so should have the same index $\mathcal{I}_{W_{1}}=\mathcal{I}_{W_{2}}$ whenever $W^{\text {asymp }}(X)$ is nondegenerate. Therefore we find again that

$$
\mathcal{I}_{W}=0
$$

outside codimension-one walls, where $W^{\text {asymp }}(x)$ is degenerate, in the parameter space of $W$, bringing us back to $(2.50)$.

The Lefschetz fixed point theorem. When the theory admits a global symmetry, the index theorem can be substantially simplified into a form of the Lefschetz fixed point theorem [10]. Introducing external vector multiplets $A_{i}$ that couple to such global symmetries, the zero modes thereof, $a_{i}$, enter the Hamiltonian, schematically, as follows:

$$
H \rightarrow H(a)=H+\cdots+a_{0} F,
$$


where $F$ is the global symmetry charge. The ellipsis denotes complex mass terms due to $a_{1,2}$ while the combination $\beta a_{0}$ will play the role of the chemical potential.

In turn, $F$ will have the form

$$
-F=\mathcal{L}_{F}+\text { fermion bilinear }
$$

where $\mathcal{L}_{F}$ rotates the bosonic variables. When we proceed with the Heat kernel expansion the latter has the effect of

$$
\left\langle X\left|e^{-\beta H(a)}\right| X\right\rangle=\left\langle X\left|e^{-\beta\left(H(a)+a_{0} \mathcal{L}_{F}\right)}\right| X\left(\beta a_{0}\right)\right\rangle,
$$

where $X\left(\beta a_{0}\right)=e^{\beta a_{0} \mathcal{L}_{F}} X$.

As we expand in small $\beta$, as in previous section, this shift creates a term in the exponent of type $\left(X\left(\beta a_{0}\right)-X\right)^{2} / \beta$, from (2.7), which in turn translates to $\sim\left(\beta a_{0} \mathcal{L}_{F}[X]\right)^{2} / \beta$ unless $a_{0}$ becomes as large as $1 / \beta$. Thus, the rotation by small but finite $\beta a_{0}$ makes fields that rotate under $F$ arbitrarily massive. Since the bosonic and the fermionic degrees of freedom match precisely, their contributions cancel out, leaving behind the invariant loci only. In the end, one merely collect the field content that spans the invariant locus and use (2.32) for this subsystem.

If the dynamics has no asymptotic flat directions, the usual topological robustness of index ensures that the addition of this mass term will not affect the outcome $\mathcal{I}=\Omega$. As such, the index theorem further reduces to a sum of smaller index problems, each of which is defined over the common invariant loci of all global symmetries. Labeling these loci by $l$, we find

$$
\Omega=\sum_{l} \Omega(l)
$$

where $\Omega(l)$ is the Euler index of the locus $l$.

In particular, it is worthwhile to note that the "flavor chemical potential" $a_{0}$ does not appear anywhere in the final expression. This is in a stark contrast against the localization computation with complex supersymmetries in recent literatures. This appears to be related to the fact that with our minimal supersymmetry there is no $R$-symmetry chemical potential available. Indeed for many of existing twisted partition functions with larger supersymmetries, such as the $(2,2)$ elliptic genus and the refined index for $\mathcal{N}=4$ quantum mechanics, the dependence on flavor chemical potentials tends to disappear altogether as one takes the limit of the vanishing $R$-charge chemical potential.

When asymptotic and gapped flat directions are present in the original problem but entirely lifted by this mass deformation, (2.32) will naturally compute the desired index $\mathcal{I}$ instead of $\Omega$. If such a lift is not quite complete, (2.32) will again compute some $\Omega$, from which reading off $\mathcal{I}$ is again a matter of scaling $W$ by a large positive number. If there are gapless asymptotic flat directions in the original problem, the resulting $\Omega$ computed by our fixed point theorem needs to be treated more carefully; for one thing, the original theory might be sitting at a wall of marginal stability, in which case the mass deformation of this kind may not be very innocuous. 
Again with the multi-scalar prototype of the previous subsection, even $n$ admits $n / 2$ $\mathrm{SO}(2)$ 's that rotate $X$ 's pairwise, say $X_{1}$ and $X_{2}, X_{3}$ and $X_{4}$, etc. The invariant locus is $\vec{X}=0$, where $\operatorname{det}(\partial \partial W)=0$. Once the path integral localizes to such a locus, $\Omega$ vanishes, and so we find $\mathcal{I}=0$ as well. For odd $n,(n-1) / 2 \mathrm{SO}(2)$ rotation kills all $X$ 's but the last: hence the computation reduces, effectively, to $n=1$, and we again find $\mathcal{I}=2$ in the end.

A word of caution is needed here. Although the Lefschetz fixed point theorem is applicable to theories with compact dynamics, one also encounters examples where the dynamics prior to turning on the chemical potential and the real mass is not compact while, thereafter, it becomes compact. In such situation, the index computed via the fixed point theorems will be integral, yet it is not obvious what it computes. In many localization computations with larger supersymmetries, the chemical potentials do survive in the final form of the twisted partition functions, yet one usually encounters a pole at the $a=0$ limit, giving us an ample warning in interpreting such results. For the generalized Euler index of ours, such poles are absent since $a$ itself disappears in the end; it remains to be seen if this independence implies no adverse contamination of $\mathcal{I}$ by such localizing masses or merely misleading results.

\subsection{Wall-crossing}

The wall-crossing phenomenon, familiar from $\mathcal{N}=4$ gauged quantum mechanics, is discontinuity of the (refined) index which occurs as one continuously changes FI constants. With the current $d=1 \mathcal{N}=2$ real supersymmetries, the auxiliary $D$ field of the $d=1$ $\mathcal{N}=4$ vector multiplet now becomes an auxiliary and real $F$ field of the real scalar multiplet. This means that the wall-crossing would be ubiquitous in $d=1 \mathcal{N}=2$ theories, as one deforms the superpotential, with or without the gauge sector. Here we wish to characterize the wall-crossing phenomena in the absence of the gauge sector, in terms of the superpotential $W$. We will find that the basic path-integral form (2.32) for $\Omega$ and the winding number interpretation (2.48) are most useful for understanding the wall-crossing in this reduced setting.

On the other hand, the topological robustness of the index must still hold; as such the index should remain piecewise constant in the space of the superpotential and can change only when we change the asymptotics of $W$ qualitatively. We will mostly work with a polynomial form of $W$, and characterise how the primary wall-crossing happens even while we deform the leading homogeneous part, call it $W^{(p+1)}$, not necessarily changing the power. This is different from $\mathcal{N}=4$ Landau-Ginzburg theories where the leading power of the holomorphic $W$ fixes the index once and for all.

The simplest example of wall-crossing occurs with the quadratic $W$. Suppose we have

$$
W(X)=\frac{1}{2} \sum_{\mu, \nu} m_{\mu \nu} X^{\mu} X^{\nu}
$$

If $m$ is non-singular, the superpotential admits a single critical point $\vec{X}=0$, and as such one immediately learns that $|\mathcal{I}|=1$. While the sign of $\mathcal{I}$ is in principle ambiguous for a given theory due to the same ambiguity with $(-1)^{\mathcal{F}}$, the relative sign of $\mathcal{I}$ between a pair of theories that can be continuously connected is not ambiguous. 
It is clear that the sign of $\mathcal{I}$ is dictated by the fermionic Pfaffian, or in this case the determinant of $m$, so that ${ }^{3}$

$$
\mathcal{I}=\operatorname{sgn}(\operatorname{det}(m)) .
$$

As is clear from the index formula (2.32), the wall-crossing here is from how the map

$$
X^{\mu} \rightarrow m_{\mu \nu} X^{\mu}
$$

reverses the orientation as one crosses codimension-one wall $\operatorname{det}(m)=0$ in the space of the mass matrix. at a point where exactly one eigenvalue of $m$ vanishes. At such $m$, the superpotential becomes degenerate and a flat and gapless asymptotic direction opens up, pushing the vacuum to infinity. In this simple prototype, one can see that a vacuum is pushed out to infinity as one approaches such a wall, while on the other side of the wall a different vacuum with the opposite chirality moves in from the infinity. If one jumps across two such walls, the two wall-crossing would cancel each other. Therefore, the wall-crossing in this simple toy model is such that $\operatorname{det}(m)=0$ divides the parameter space into two regions, each consisting of many disconnected wedges, for which $\mathcal{I}=1$ or $\mathcal{I}=-1$.

What happens if we allow subleading linear terms? With

$$
W(X)=\frac{1}{2} \sum_{\mu, \nu} m_{\mu \nu} X^{\mu} X^{\nu}+\sum_{\mu} k_{\mu} X^{\mu},
$$

what changes qualitatively for wall-crossing? When $\operatorname{det}(m) \neq 0$ the asymptotics are dominated entirely by $m_{\mu \nu}$, so linear terms do little. To see what happens at the wall $\operatorname{det}(m)=0$, we diagonalize the quadratic term and re-label the fields

$$
W(X, Y)=\frac{1}{2}\left(m_{1}\left(X_{1}\right)^{2}+m_{2}\left(X_{2}\right)^{2}\right)+k_{1} X_{1}+k_{2} X_{2},
$$

so that the vacuum conditions are

$$
m_{1} X_{1}+k_{1}=0, \quad m_{2} X_{2}+k_{2}=0 .
$$

When one of the eigenvalues vanishes, say $m_{2}=0$, the vacuum again runs off to infinity, $X_{2}=-k_{2} / m_{2} \rightarrow \infty$. Note that this could not be seen clearly if we sat at $m_{2}=0$; one sees only that no finite vacuum exists. If $k$ 's were absent, this runaway vacuum phenomenon would have been manifested as a flat gapless direction. However, with or without $k$ 's the end result for $\mathcal{I}$ is the same: a wall appears when the leading quadratic terms become degenerate, and a codimension-one wall-crossing occurs across such a wall.

This simple pattern is really a universal feature of codimension-one wall-crossings for polynomial $W$. Consider

$$
\begin{aligned}
W(X) & =W^{(p+1)}(X)+W^{(p)}(X)+\cdots, \\
W^{(q)}(X) & =\sum_{\sum n_{I}=q} C_{n_{1} \cdots n_{N}}^{(q)} X_{1}^{n_{1}} \cdots X_{N}^{n_{N}},
\end{aligned}
$$

\footnotetext{
${ }^{3}$ We have in effect fixed the would-be ambiguous sign convention for $(-1)^{\mathcal{F}}$, in favor of this sign choice, and will follow the same convention throughout this note.
} 
with coefficients $C$ 's assumed to be generic. Since a flat gapless asymptotic direction or a runaway vacuum is necessary, the wall-crossing is possible if and only if the leading homogeneous $W^{(p+1)}(X)$ admits a nonzero critical point. Due to the scaling $W^{(p+1)}(\lambda X)=$ $\lambda^{p+1} W^{(p+1)}(X)$, a nonzero solution to $0=\vec{\partial} W^{(p+1)}(X)$ generates an $\mathbb{R}$ 's worth of vacua of $W^{(p+1)}(X)$. This means that a codimension-one wall-crossing occurs precisely at parameters where $W^{(p+1)}(X)$ becomes degenerate, or in other words, when

$$
d W^{(p+1)}=0
$$

admits a straight line of solutions passing through the origin and extending out to the asymptotic region. Note that the homogeneity implies $W^{(p+1)}=0$ as well. When we take into account subleading terms, the same codimension-one condition can instead translate to a vacuum that runs away to infinity, and the location of these codimension-one walls are not affected by such subleading terms in $W$.

It is important to note here that the codimension-one walls defined by the leading power $W^{(p+1)}$ of $W$ are invariant under the overall scaling, such that the homogeneous rescaling of the coefficients, power by power, does not change the index. This observation is important since we performed scalings of coefficients in $W$, different for different powers of $X$ 's, to reach at $w$ with the same functional form as $W$. Under such power-dependent scalings of coefficients, no wall-crossing happens as long as the leading power remains nondegenerate in the above sense. This justifies the main result of this section, (2.32), despite potential wall-crossing threats.

Recall that, away from the codimension-one walls, the index $\mathcal{I}$ is also entirely determined by the asymptotic superpotential, $W^{(p+1)}$, as is obvious from the winding number interpretation (2.48). Sometimes, this by itself produces indices and wall-crossing rather quickly. For example, consider theories with two scalar multiplets, starting with a cubic model,

$$
W^{(3)}=\operatorname{Re}\left(A_{0} \Phi^{3}+A_{1} \Phi^{2} \bar{\Phi}\right)
$$

with $\Phi=\left(X_{1}+i X_{2}\right) / \sqrt{2}$ and complex numbers $A_{0}$ and $A_{1}$. The effective number of times $d W^{(3)}$ covers $\tilde{\mathbb{R}}^{2}$ equals the winding number of

$$
\left(\partial_{1}+i \partial_{2}\right) W^{(3)}=3 \bar{A}_{0} \bar{\Phi}^{2}+2 \bar{A}_{1} \Phi \bar{\Phi}+A_{1} \Phi^{2}
$$

which says immediately that the absolute value of the winding number cannot exceed 2 , although the number of critical points can be as many as 4 . With $\Phi \rightarrow R e^{i \phi}$ and $\phi_{1}$ the phase angle of $A_{1}$,

$$
\left(\partial_{1}+i \partial_{2}\right) W^{(3)} \rightarrow R^{2}\left[3 \bar{A}_{0} e^{-2 i \phi}+\bar{A}_{1}\left(2+e^{2 i \phi+2 i \phi_{1}}\right)\right] .
$$

$\left|A_{0}\right| \gg\left|A_{1}\right|$ results in $\mathcal{I}=-2$ while $\mathcal{I}=0$ when $\left|A_{0}\right| \ll\left|A_{1}\right| . \mathcal{I}=2$ is not allowed as the first term in the round parenthesis always dominates over the second. ${ }^{4}$ One can generalize

${ }^{4}$ The same can be seen from the Morse counting with, after an additive shift of $X$,

$$
W\left(X_{1}, X_{2}\right)=\frac{1}{3} f\left(X_{1}\right)^{3}+g X_{1}\left(X_{2}\right)^{2}+\frac{1}{3} h\left(X_{2}\right)^{3}+\cdots .
$$


this to higher order polynomials,

$$
W^{(p+1)}=\operatorname{Re}\left(A_{0} \Phi^{p+1}+A_{1} \Phi^{p} \bar{\Phi}+\cdots+A_{\lfloor(p+1) / 2\rfloor} \Phi^{p+1-\lfloor(p+1) / 2\rfloor} \bar{\Phi}^{\lfloor(p+1) / 2\rfloor}\right) .
$$

The same reasoning as above tells us that the possible values of the index in the domains separated by codimension-one walls are

$$
\mathcal{I}=-p,-p+2, \ldots,-p+2\lfloor(p+1) / 2\rfloor,
$$

up to a convention-dependent overall sign. Note that the absolute value of the index, $|\mathcal{I}|$, is bounded above by $p$ which is the value in the holomorphic limit, $A_{k>0}=0$. Note that, in contrast, the maximum number of non-degenerate critical points for real $W^{(p+1)}+\cdots$ is a much larger value $p^{2}$.

What happens when we extend these to a larger number of matter multiplets? For odd $N$, two general features one can find are that $\mathcal{I}=0$ for odd $p+1$, as already shown in the section 2.4 , and that, for even $p+1$,

$$
\mathcal{I}=-2 k+1,-2 k+3, \cdots, 2 k-3,2 k-1,
$$

for some integer $k>0$. For $p+1=2$, one can see $k=1$ either from the Morse counting or from the winding; e.g., the map $X \rightarrow d W^{(2)}$ is linear, so can have at most \pm 1 winding number, immediately leading us to $\mathcal{I}= \pm 1$. The sign-symmetric nature can be argued easily by considering $W^{(p+1)}$ and $-W^{(p+1)}$ pairwise. For even $N$, no systematic feature appears; for example, the upper bound on $|\mathcal{I}|$ is no longer set by the holomorphic limit for even $N$. Leaving aside all such complicated patterns, however, the wall-crossing pattern itself appears to be very simple: for each codimension-one wall, dictated by the coefficients of the leading power of the polynomial $W$, the general pattern of the wall-crossing is very simple with

$$
\Delta \mathcal{I}= \pm 2
$$

which generalizes (2.20) for a single scalar multiplet or the pattern seen in (2.69) of the two scalar multiplet theories. Generically, a Morse vacuum moves off to the asymptotic infinity, only to return on the other side of the wall with the opposite chirality.

Although we confined our attention to codimension-one walls of marginal stability, it is not difficult to see that there can be more complicated patterns of the index when one sits at a codimension-one wall, however. The leading $W^{(p+1)}$ becomes degenerate at such a wall, meaning that for some asymptotic directions, the subleading pieces $W^{(q \leq p)}$ can now alter the asymptotic behavior qualitatively. In other words, when $W^{(p+1)}$ sits on a codimension-one wall, $\vec{\partial} W^{(p+1)}$ passes through the origin such that small "subleading"

More explicitly, the codimension-one walls are at $f=0$ and $h^{2} f+4 g^{3}=0$ such that

$$
\begin{array}{ll}
\mathcal{I}=-2 & 0<h^{2} f<-4 g^{3} \text { or }-4 g^{3}<h^{2} f<0, \\
\mathcal{I}=0 & 0<-4 g^{3}<h^{2} f \text { or } h^{2} f<-4 g^{3}<0 .
\end{array}
$$


corrections can alter the winding number qualitatively. This means that there are actually a nested network of marginal stability walls. In this note, however, we will not consider such possibilities. When we lift the situation to $d=3$ minimally supersymmetric theories, for example, there is no non-renormalization theorem that protects the superpotential, so a fine-tuning of $W$ does not make much sense. At least physically there seems to be no reason for a theory to sit on such a tightly constrained place in the parameter space.

\subsection{Uplifts to $d=2,3$}

What happens if we try to extend the computation here straightforwardly to higher dimensions? One should start by putting $d$ dimensional theory on $\mathbb{T}^{d}$ torus. The supersymmetry content matches two real fermions for one real boson, so that the determinants of massive sector cancel precisely due to supersymmetry regardless of the spacetime dimensions. This precise cancelation is further reinforced by the absence of $R$-charge that commutes with a supercharge, and as such the Kaluza-Klein modes cancel out precisely. This means that, as long as we consider massive theories with no continuum of classical supersymmetric vacua, our computation and the index theorem are applied to higher $d=2,3$ verbatim. On the other hand, if one starts with $d=3$ theory with continuum of vacua, the Witten index is ill-defined to begin with, so only upon some massive deformations that can lift such continua one can discuss the Witten index. For such theories, again our $d=1$ approach will work verbatim once the theory is put on $\mathbb{T}^{3}$.

Since the wall-crossing phenomenon arises from the asymptotic flat directions in the matter sector, the wall-crossing uplifts straightforwardly to higher dimensions as well. This should be contrasted to the more familiar wall-crossing of $d=1 \mathcal{N}=4$ theories, which is essentially a Coulomb phase physics and does not uplift to $d>1$. So, again assuming that we deal with massive theories, our discussion of the wall-crossing will apply straightforwardly to $d=2 \mathcal{N}=(1,1)$ theories and to $d=3 \mathcal{N}=1$ theories. In fact, the wall-crossing has been observed directly for some $d=3 \mathcal{N}=1$ theories [25].

New ingredients that distinguish different spacetime dimensions will manifest once we consider gauge theories: the main differences arise from the would-be zero modes associated with the gauge fields, namely the gauge holonomies. In next two sections, we will explore the smallest of gauged dynamics in $d=1$ and $d=3$ settings, which will give us some further understanding of how vacuum counting in these two different spacetime dimensions share some common features and how they differ from each other. In the end, we will find index formulae for $d=1 \mathcal{N}=2 \mathrm{SO}(2)$ theories and for $d=3 \mathcal{N}=1 \mathrm{SO}(2)$ gauge theories, which are not that different from (2.32) at least in spirit.

\section{$3 d=1 \mathcal{N}=2$ abelian gauge theories}

Gauging requires further steps. For the same reason as the scalar multiplet theories, the massive modes cancel out precisely in the path integral. The zero mode is another matter. The gauge zero modes $a_{i}$ 's should be integrated over, and so are their partner gaugini, which alter the procedure qualitatively. Also the trace over the Hilbert space must be done 
for the gauge invariant states only, so we include the projection operator $\mathcal{P}_{\mathcal{G}}$

$$
\operatorname{Tr}(-1)^{\mathcal{F}} e^{-\beta \mathcal{H}} \mathcal{P}_{\mathcal{G}},
$$

where the projection operator is given by

$$
\mathcal{P}_{\mathcal{G}}=\frac{1}{\operatorname{vol}(\mathcal{G})} \int_{\mathcal{G}} d^{g} \Theta e^{i \Theta \cdot G}
$$

with the Gauss constraint $G$ and $g=\operatorname{dim}(\mathcal{G})$. This results in the normalization of the measure for the time-like gauge zero modes, $\sim \Theta / \beta$, that is a little different from its space-like counterpart, as we see below.

In particular, while the standard $\beta \rightarrow 0$ scaling seemingly reduces $\Theta$ into $\mathbb{R}^{g}$-valued, replaced by a gauge-field along the time direction, its periodic nature must not be forgotten. How this periodic origin should enter the index quantitatively has been only very recently understood [20,21], surprisingly enough. We will outline it in the next subsection and encounter it repeatedly in the rest of this note. The same observation will play an essential role when we uplift the story to $d=3$ later, where we will compute $d=3$ Witten index from a $d=1$ perspective.

Another issue for gauge theories is the potentially flat asymptotic direction along the Coulomb phase. Such new flat gapless directions can potentially complicate the problem further in two essential ways. The first is how $\Omega$ could generically become non-integral due to the continuum contribution; the second is potential additional wall-crossing if such flat gapless directions appear during a continuous deformation. We will encounter some of these issues with concrete examples below. However, it is worthwhile to note that the connection to $d=3 \mathcal{N}=1$ theories mitigates some of these complications. One unexpected result is that $\Omega$ of a $d=1$ theory obtained from dimensional reduction of $d=3 \mathcal{N}=1$ theory does not actually experience wall-crossing due to asymptotically gapless Coulomb directions, contrary to the general wisdom.

The first problem of non-integral value of $\Omega$ persists, on the other hand. In particular we will find that $\Omega$ in such theories is a multiple of $1 / 2$ in all examples we discuss. In strict $d=1$ problems, computing $\mathcal{I}$ in such situations is not straightforward. With larger supersymmetries, the continuum part of such non-integral results have been studied rather generally in recent years and in some cases classified and understood to the extent that the extraction of $\mathcal{I}$ is possible [19]. For the current problems, we do not have such a machinery. On the other hand, when we move on to $d=3$ Chern-Simons theories, where as noted above there are no longer Coulombic continuum, this non-integral contribution will be a seed to a very well-known interpretation as the half-integral shift of the Chern-Simons level due to massive charged fermions. We come back to this in section 4 .

\section{1 $\Omega$ for gauge theories and holonomy saddles}

The analog of (2.6) for gauge theories is, at least naively,

$$
\lim _{\beta \rightarrow 0^{+}} \frac{\beta^{N / 2+g}}{(2 \pi)^{N / 2+g_{\operatorname{Vol}}(\mathcal{G})}} \int d^{N} X d^{3 g} \mathbf{u} \operatorname{Pf}(M(X, \mathbf{u})) e^{-\beta V_{\text {bosonic }}(X, \mathbf{u})},
$$


where $N$ is the number of real scalar multiplets and $\mathbf{u}$ denotes collectively the $3 g$ number of constant bosonic modes from the vector multiplet. According to the convention to count real multiplets, $N_{f}$ matters charged to the $\mathrm{SO}(2)$ gauge group and $\operatorname{dim}(S)$ singlets lead to $N=2 N_{f}+\operatorname{dim}(S)$. The prefactor of the integrand arises from the fermionic zero-mode integral of $N+g$ copies of the fermion bilinear; It is the Pfaffian of a rank $2(N+g)$ matrix

$$
M(X, \mathbf{u})=\left(\begin{array}{cc}
u_{i}^{r}\left(T_{r}^{\mathrm{adj}}\right)_{p q}\left(\epsilon \cdot \sigma^{i}\right)_{a b} & \left(T_{q} X\right)_{\nu} \epsilon_{a c} \\
\left(X T_{p}^{\dagger}\right)_{\mu} \epsilon_{d b} & \partial_{\mu} \partial_{\nu} W \epsilon_{d c}+u_{i}^{r}\left(T_{r}\right)_{\mu \nu}\left(\epsilon \cdot \sigma^{i}\right)_{d c}
\end{array}\right)
$$

where $T_{p}$ is the representation of the Lie Algebra of $\mathcal{G}$ on $X$ 's, and $T_{r}^{\text {adj }}$ the adjoint representation. Note that in addition to the spatial zero modes $u_{1,2}$, its time-like counterpart arises as $u_{0} \equiv \Theta / \beta$, which means that on the right hand side of (3.3) the integration over $\Theta$ is now converted to a vanishingly small neighborhood of $\Theta=0$. This is a somewhat trivial example of the holonomy saddle, to which we come back below.

For now, let us see how the factor $\beta$ can be removed from the expression. The bosonic potential has three types of terms, $\sim(\partial W)^{2}, \sim \mathbf{u}^{2} X^{2}$, and $\sim \mathbf{u}^{4}$. Unless $W$ is purely cubic there is no scaling of variables that remove the overall factor $\beta$ cleanly from the exponent. However, since $W$ itself can be scaled without affecting the genuine index $\mathcal{I}$, we may as well introduce

$$
X \leftarrow \beta^{1 / 4} X, \quad \mathbf{u} \leftarrow \beta^{1 / 4} \mathbf{u},
$$

and in addition scale $\beta^{3 / 4} W \rightarrow \tilde{W}$, so that

$$
\partial \tilde{W} \leftarrow \beta^{1 / 2} \partial W
$$

is kept finite as a function of $\beta$-rescaled $X$. This in particular demands appropriate scalings of coefficients in $W$. Only if $W$ is purely cubic in $X$, no separate scaling of $W$ is necessary. As we have already noted for matter-only theories, however, such scaling is harmless away from codimension-one walls of marginal stability.

In terms of the newly rescaled quantities, we have

$$
M=\beta^{-1 / 4} \tilde{M}
$$

where $\tilde{M}$ denotes the same matrix but with the rescaled $X$ 's and $\mathbf{u}$ in places of the original $X$ 's and $\mathbf{u}$ and with $\tilde{W}$ in place of $W$. Hence we have

$$
\operatorname{Pf}(M)=\beta^{-(N+g) / 4} \operatorname{Pf}(\tilde{M}),
$$

which cancels the factor of $\beta$ left over after absorbing $\beta^{1 / 4}$ 's to the integral variables

$$
\beta^{N / 2+g} \int d^{N} X d^{3 g} \mathbf{u} \rightarrow \beta^{(N+g) / 4} \int d^{N} X d^{3 g} \mathbf{u}
$$

precisely. Therefore, we find

$$
\frac{1}{(2 \pi)^{N / 2+g_{\operatorname{Vol}}(\mathcal{G})}} \int d^{N} X d^{3 g} \mathbf{u} \operatorname{Pf}(\tilde{M}) e^{-\tilde{V}_{\text {bosonic }}(X, \mathbf{u})},
$$


which brings the path integral to a finite-dimensional one. Throughout this note, when the integral involves no factor of $\beta$ explicitly, the variable should be understood as those rescaled.

Most generally, however, there could be further additive contributions to $\Omega$, similar to (3.3) but from the expansion of the gauge variables of type $\Theta=\Theta_{*}+\beta u_{0}$ where $e^{i \Theta_{*} \cdot G} \neq 1$. One thus finds a sum over $\Theta_{*}$ 's,

$$
\Omega=\sum_{\Theta_{*}} d_{H} \mathcal{Z}_{H}
$$

where $\mathcal{Z}_{H}$ is the partition function of the low-lying theory $H$ at $\Theta_{*}$, analogous to the right hand side of (3.10) but for a different theory $H$, while the factor $d_{H}$ is from integrating out heavy modes of masses $\sim 1 / \beta$. Each such $\Theta_{*}$ contribution arises from a vanishingly small local neighborhood around $\Theta_{*}$ in the small $\beta$ limit, and (3.10) is merely one such at $\Theta_{*}=0$.

The criterion for the contributing $\Theta_{*}$ is that the low energy theory $H$ there has nonzero partition function $\mathcal{Z}_{H}$. This in turn requires that the light sector sitting at such a point $\Theta_{*}$ admits no decoupled fermions [20]. For example, one obvious class of $\Theta$ 's which cannot be a contributing saddle are those holonomies where the light theory $H$ has a decoupled Abelian gauge sector; the resulting free gaugini which kill any reasonable form of the partition function $\mathcal{Z}$ there in small $\beta$ limit. At generic $\Theta$, all charged field content tends to acquire a heavy mass of order $\Theta / \beta$, leaving behind the free Cartan sector, which suffices to tell us that the contributing $\Theta_{*}$ would occur at most discretely.

Such pairs $\left(\Theta_{*}, H\right)$ have been dubbed the holonomy saddles in the context of supersymmetric gauged quantum mechanics [20] as well as for various partition functions of supersymmetric gauge theories in higher dimensions [21]. Although this phenomenon persists in all spacetime dimensions, it takes a particularly simple form for $d=1$. In the latter $d_{H}$ reduces to a numerical factor whereas, in higher spacetime dimensions, it can carry further nontrivial physics such as the Cardy exponent [21, 22].

A simplest example of the non-trivial holonomy saddles can be found with an $\mathrm{SO}(2)$ theory with both unit-charged and double-charged matter multiplets. $\Theta_{*}=0$ is the naive saddle where the theory $H$ has the same field content as the original theory. The other holonomy saddle sits at $\Theta_{*}=\pi$, where the $H$ theory is again an $\mathrm{SO}(2)$ theory but with double-charged matter only, and $d_{H}$ would be from integrating out the unit-charged matter. For other values of $\Theta$, the would-be $H$-theory consists of a pure $\mathrm{SO}(2)$ gauge theory, whose $\mathcal{Z}_{H}$ vanishes identically due to the decoupled gaugino. Thus the holonomy saddles of this simple theory consist of $\Theta_{*}=0$ and $\Theta_{*}=\pi$. Likewise, if an $\mathrm{SO}(2)$ theory admits a charge $q$ scalar multiplet, we will find holonomy saddles at $\Theta_{*}=2 \pi n /|q|$ for $n=0,1, \cdots,|q|-1$.

Another special class of holonomy saddles is when one finds $\Theta_{*} \neq 0$ but $e^{i \Theta_{*} \cdot G}$ acts on the entire field content trivially. Each such saddle contributes the same amount as the canonical $\Theta_{*}=0$ saddle in (3.3). The net effect of these can be incorporated as a numerical multiplicative factor in front of expressions like (3.3). Alternatively, the same effect is achieved by using the version of $\mathcal{G}$ divided by the subgroup defined by a collection of such mutually equivalent holonomies. For such theories in $d=1$, the net effect of these 
can be achieved by resorting to (3.3) but taking care to choose $\mathcal{G}$ in (3.3) to be "minimal" by modding out by any discrete subgroup that acts trivially on the field content. For instance, if charged matter $X$ is absent, $\mathcal{G}$ would be the one divided by the center. For $d>1$, however, such shortcut is no longer valid because the multiplicity of such holonomy saddles depends on spacetime dimension. As such, we must honestly evaluate the sum (3.11).

\subsection{A prototype: $\mathrm{SO}(2)$ with massive charged matters}

As a prototype, let us consider a simple $\mathrm{SO}(2)$ theory, now with $N_{f}$ massive real twocomponent charged scalars $\vec{X}_{I}=\left(X_{I(1)}, X_{I(2)}\right)$ rotating in a pair, with charge $q_{I}$ under the $\mathrm{SO}(2)$, or equivalently $q_{I}$-charged $N_{f}$ complex scalar multiplets $\Phi_{I}=\left(X_{I(1)}+i X_{I(2)}\right) / \sqrt{2}$. Let us take the superpotential,

$$
W=\frac{1}{2} \sum_{I=1}^{N_{f}} m_{I}\left|\vec{X}_{I}\right|^{2}
$$

The $\beta$-scaled bosonic potential and Pfaffian are given by

$$
\begin{aligned}
\tilde{V}_{\text {bosonic }} & =\sum_{I=1}^{N_{f}} \frac{1}{2}\left(m_{I}^{2}+q_{I}^{2} \mathbf{u}^{2}\right)\left|\vec{x}_{I}\right|^{2}, \\
\operatorname{Pf}(\tilde{M}) & =\sum_{I=1}^{N_{f}}\left[\prod_{J \neq I}^{N_{f}}\left(m_{J}^{2}+q_{J}^{2} \mathbf{u}^{2}\right)\right] m_{I} q_{I}^{2}\left|\vec{x}_{I}\right|^{2} .
\end{aligned}
$$

Although this example can be integrated straightforwardly, we take an alternate route that will be useful for generalizing to more general field content and the superpotential.

Let us define

$$
\vec{y}_{I}=\sqrt{m_{I}^{2}+q_{I}^{2} \mathbf{u}^{2}} \cdot \vec{x}_{I}
$$

whereby the bosonic potential is completely written as the sum of their squares. With the accompanying Jacobian

$$
\prod_{I=1}^{N_{f}}\left(m_{I}^{2}+q_{I}^{2} \mathbf{u}^{2}\right)^{-1}
$$

we arrive at

$$
\Omega^{\Theta_{*}=0}=\frac{1}{(2 \pi)^{N_{f}+2}} \int d^{3} \mathbf{u} \sum_{I=1}^{N_{f}} \frac{m_{I} q_{I}^{2}}{\left(m_{I}^{2}+q_{I}^{2} \mathbf{u}^{2}\right)^{2}} \int d^{2 N_{f}} y\left|\vec{y}_{I}\right|^{2} e^{-\sum_{J=1}^{N_{f}} \frac{1}{2}\left|\vec{y}_{J}\right|^{2}},
$$

where the matter sector and the gauge sector become factorized. The universal gauge sector, parameterized by variables $\mathbf{u}$, gives

$$
\int_{\mathbb{R}^{3}} d^{3} \mathbf{u} \frac{m_{I} q_{I}^{2}}{\left(m_{I}^{2}+q_{I}^{2} \mathbf{u}^{2}\right)^{2}}=\pi^{2} \frac{m_{I}}{\left|m_{I}\right|} \frac{1}{\left|q_{I}\right|}
$$

so that we end up with

$$
\Omega^{\Theta_{*}=0}=\frac{1}{2} \sum_{I=1}^{N_{f}} \frac{m_{I}}{\left|m_{I}\right|} \frac{1}{\left|q_{I}\right|} .
$$


This simple trick needs to be refined for more general theories, but this suffices as an illustration for now.

With $\left|q_{I}\right|>1$, the holonomy saddles enter the story, which is why we labeled the above by $\Theta_{*}=0$. The additional saddles appear at $\Theta_{l}=\frac{2 \pi l}{\left|q_{I}\right|}$ for $l=1, \ldots,\left|q_{I}\right|-1$, since the holonomy saddles are given by

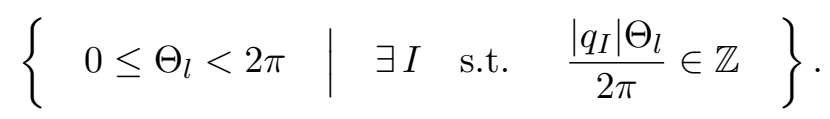

At the saddle $\Theta_{l}$, only the matters whose charge satisfies a condition $\left|q_{I}\right| \Theta_{l} \in 2 \pi \mathbb{Z}$ contribute to the partition function. Thus, the contribution from $\Theta_{l}$ is

$$
\Omega^{\Theta_{l}}=\frac{1}{2} \sum_{I=1}^{N_{f}} \delta_{\frac{\left|q_{I}\right| \Theta_{l}}{2 \pi}} \frac{m_{I}}{\left|m_{I}\right|} \frac{1}{\left|q_{I}\right|},
$$

where $\delta_{\frac{\left|q_{I}\right| \Theta_{l}}{2 \pi}}$ is defined by

$$
\delta_{\frac{\left|q_{I}\right| \Theta_{l}}{2 \pi}}= \begin{cases}1, & \left|q_{I}\right| \Theta_{l} \in 2 \pi \mathbb{Z}, \\ 0, & \text { else } .\end{cases}
$$

It satisfies the following condition

$$
\sum_{l} \delta_{\frac{\left|q_{I}\right| \Theta_{l}}{2 \pi}}=\left|q_{I}\right|
$$

because each matter multiplet appears $\left|q_{I}\right|$ times over the entire holonomy saddles. Therefore, the total twisted partition function is given by

$$
\Omega=\sum_{l} d_{l} \Omega^{\Theta_{l}}=\frac{1}{2} \sum_{I=1}^{N_{f}} \operatorname{sgn}\left(m_{I}\right) .
$$

Although the holonomy saddles are not all equivalent if $q_{I} \neq q_{J}$ for some pairs, the end result is merely to multiply $\left|q_{I}\right|$ to $1 /\left|q_{I}\right|$ for each summand in (3.19).

The result in (3.24) by itself is not acceptable as the Witten index $\mathcal{I}$ of the $d=1$ theory; these fractional answer appears because of the three flat $\mathbf{u}$ directions with $E_{\text {gap }}=0$, sitting at $\vec{X}=0$. Note that, however, the answer is an integer multiple of $1 / 2$, so it cannot be some random continuum contribution either. In the final section we will make sense of it in the context of $d=3$ theories.

\subsection{Gaussian reduction}

Recall how the index for matter-only theories reduces to a pure Gaussian integral in the space of $\partial W(x)$, which can be, under a suitable condition on the asymptotic data, expressed either as the winding number of the map $x \rightarrow \partial W(x)$ or the Morse counting. Although we have found a similar change of variables for the simple example above, where the charged matter enters $W$ quadratically at most, this turns out to be not universal enough. When we begin to discuss $d=3$ index, we will make a good use of these variable choices, for an 
additive piece proportional to the Chern-Simon level. For the current $d=1$ computation, however, we need something a bit more sophisticated to turn $\Omega$ into Gaussian integrals much like (2.32).

Here, we present one such approach for general $\mathrm{SO}(2)$ gauge theory with superpotential being an arbitrary function of the "radial" variables $\rho_{I}=\left|\vec{X}_{I}\right|^{2} / 2$, and possibly of singlets $P_{A}$ 's as well. In other words, we precluded $\vec{X}_{I} \cdot \vec{X}_{J}$ term with $I \neq J$. What have we lost in doing so? Note that such a restriction would come about automatically if we impose $\mathrm{SO}(2)^{N_{f}-1}$ flavor symmetries. Such flavors, however, have been used routinely in recent supersymmetric path integral evaluations, and also by us in section 2 for a contact with the Lefschetz fixed point theorem.

The lesson from these is that restricting the Lagrangian or adding (mass) terms to reinforce such a symmetry is innocuous as long as the original dynamics is sufficiently gapped so that the usual topological robustness of index, or more precisely the twisted partition function, can be argued. As such our restriction,

$$
W=W\left(\rho_{I}, P_{A}\right)
$$

possibly with neutral $P_{A}$ 's, is well-justified as long as $d W$ is sufficiently divergent along all asymptotic directions.

With such $W\left(\rho_{I}, P_{A}\right)$, we decompose its $\beta$-scaled Pfaffian of fermion bilinear based on the recursive definition of Pfaffian

$$
\operatorname{Pf}(A)=\sum_{j=2}^{N}(-1)^{j} a_{1 j} \operatorname{Pf}\left(A_{\hat{1} \hat{j}}\right),
$$

of $N \times N$ anti-symmetric matrix $A=\left(a_{i j}\right)$, where $A_{\hat{1} \hat{j}}$ denotes $(N-2) \times(N-2)$ minor of matrix $A$ with both the first and $j$-th rows and columns removed. Then we seek a series of appropriate parameterizations for minors so that each renders the part of integrand to a Gaussian form. The Pfaffian of fermion bilinear $\tilde{M}$ of an Abelian gauge theory with $N_{f}$ doublets and $\operatorname{dim}(S)$ singlets is expanded as

$$
\operatorname{Pf}(\tilde{M})=\sum_{I}\left(Q \vec{X}_{I}\right) \cdot \operatorname{Pf}\left(\tilde{M}_{I}\right)
$$

where $\operatorname{dim}(S)$ stands for the number of singlets $P$ introduced in the theory. Recall $T_{q}$, a gauge-symmetry generator in (3.4) is replaced with $Q$, Abelian $\mathrm{SO}(2)$ gauge symmetry charge by which $\vec{X}_{I}$ transforms. In the summand, $\left(Q \vec{X}_{I}\right) \cdot \operatorname{Pf}\left(\tilde{M}_{I}\right)$ stands for a collective expression:

$$
\left(Q \vec{X}_{I}\right) \cdot \operatorname{Pf}\left(\tilde{M}_{I}\right) \equiv\left(-q_{I} X_{I(2)}\right) \cdot \operatorname{Pf}\left(\tilde{M}_{I(1)}\right)+\left(q_{I} \vec{X}_{I(1)}\right) \cdot \operatorname{Pf}\left(\tilde{M}_{I(2)}\right),
$$

where $q_{I}$ is the charge of $\vec{X}_{I}$ and $\tilde{M}_{I(j)}$ is a fermion bilinear matrix with both $\left(\lambda, \psi_{I(j)}\right)$ columns and rows removed. Apparently, a singlet $P$ does not appear in (3.27) due to its charge neutrality $Q P=0$. However, the Pfaffian depends on $P$ as the second derivative terms $\partial^{2} W$ in minor $\tilde{M}_{I(i)}$ involves $P$ in general. 
For the $I$-th doublet with $I \in\left\{1, \cdots, N_{f}\right\}$, a set of integration variables are suggested by

$$
\begin{aligned}
\mathbf{v} & \equiv\left|q_{I}\right|\left|\vec{x}_{I}\right| \mathbf{u}, \quad \vec{z}_{I} \equiv \frac{\partial W}{\partial \rho_{I}} \vec{x}_{I}, \quad s_{A} \equiv \frac{\partial W}{\partial p_{A}} \\
\text { and } \quad \vec{y}_{J} & \equiv \sqrt{q_{J}^{2} \mathbf{u}^{2}+\left(\frac{\partial W}{\partial \rho_{J}}\right)^{2}} \cdot \vec{x}_{J} \quad(\text { for } J \neq I),
\end{aligned}
$$

upon which we have

$$
\left(Q \vec{X}_{I}\right) \cdot \operatorname{Pf}\left(\tilde{M}_{I}\right)\left|\frac{\partial(\vec{x}, p, \mathbf{u})}{\partial(\vec{z}, \vec{y}, s, \mathbf{v})}\right|=\frac{1}{\left|q_{I}\right| W_{I}\left|\vec{x}_{I}\right|}=\frac{1}{\left|q_{I}\right| \operatorname{sgn}\left(W_{I}\right)\left|\vec{z}_{I}\right|}
$$

for the $\left(Q \vec{X}_{I}\right)$-led piece (3.28) of the expanded Pfaffian where $W_{I} \equiv \frac{\partial W}{\partial \rho_{I}}$. Then the bosonic potential in terms of the $I$-th set of new parametrization reads

$$
\tilde{V}_{\text {bosonic }}=\frac{1}{2} \mathbf{v}^{2}+\frac{1}{2} \vec{z}_{I}^{2}+\sum_{J \neq I} \frac{1}{2} \vec{y}_{J}^{2}+\sum_{A=1}^{\operatorname{dim}(S)} \frac{1}{2} s_{A}^{2} .
$$

The full integral formula for twisted partition function $\Omega$ is arranged by the sum of $N_{f}$ integrations with the holonomy saddle contribution counted. Assume that a holonomy saddle $\Theta=\Theta_{*}$ accommodates $N_{*}\left(\leq N_{f}\right)$ charged scalar fields $\vec{X}_{I}$, with $I \in \mathcal{N}_{*}$, a set of $N_{*}$ indices. Then the full integral formula for $d=1$ index is

$$
\Omega=\sum_{\Theta_{*}} \sum_{I \in \mathcal{N}_{*}} \Omega_{I}^{\Theta_{*}}
$$

where the $\left(Q \vec{X}_{I}\right)$-led piece of twisted partition function $\Omega_{I}^{\Theta_{*}}$ around the holonomy saddle $\Theta_{*}$ reads

$$
\Omega_{I}^{\Theta_{*}}=\frac{1}{(2 \pi)^{N_{*}+2+\frac{1}{2} \operatorname{dim}(S)}} \int d^{\operatorname{dim}(\mathcal{S})} s d^{3} \mathbf{v} d^{2\left(N_{*}-1\right)} y d^{2} z_{I} \frac{1}{\left|q_{I}\right| \operatorname{sgn}\left(W_{I}\right)\left|\vec{z}_{I}\right|} e^{-\tilde{V}_{\text {bosonic }}^{*}}
$$

Note the bosonic potential $\tilde{V}_{\text {bosonic }}^{*}$ in the exponential is given by

$$
\tilde{V}_{\text {bosonic }}^{*}=\frac{1}{2} \mathbf{v}^{2}+\frac{1}{2} \vec{z}_{I}^{2}+\sum_{\substack{J \neq I \\ J \in \mathcal{N}_{*}}} \frac{1}{2} \vec{y}_{J}^{2}+\sum_{A=1}^{\operatorname{dim}(S)} \frac{1}{2} s_{A}^{2}
$$

Armed with this, let us briefly revisit the example discussed in section 3.2 as well as other examples with higher order terms in $W$.

$\boldsymbol{N}_{\boldsymbol{f}}$ massive charged matters. With this new change of variables, let us recompute the $\mathrm{SO}(2)$ theory with charged massive fields. A quadratic superpotential $W=\sum m_{I} \rho_{I}=$ $\sum \frac{1}{2} m_{I}\left|\vec{X}_{I}\right|^{2}$ gives

$$
W_{I}=m_{I}, \quad W_{I, J}=0 \quad \text { for any } I, J \in\left\{1,2, \cdots, N_{f}\right\} .
$$


A partial contribution to the twisted partition function is

$$
\Omega_{I}^{(\Theta=0)}=\frac{\operatorname{sgn}\left(m_{I}\right)}{(2 \pi)^{N_{f}+2}\left|q_{I}\right|} \int d^{3} \mathbf{v} d^{2\left(N_{f}-1\right)} y d^{2} z_{I} \frac{1}{\left|z_{I}\right|} e^{-\frac{1}{2} v^{2}-\frac{1}{2} z_{I}^{2}-\sum_{J \neq I} \frac{1}{2} \vec{y}_{J}^{2}}=\frac{1}{2} \frac{\operatorname{sgn}\left(m_{I}\right)}{\left|q_{I}\right|} .
$$

The holonomy saddles with some $\left|q_{I}\right|>1$ mean effectively a multiplicative factor of $\left|q_{I}\right|$ for $\Omega_{I}^{(\Theta=0)}$, reproducing (3.24),

$$
\Omega=\sum_{I=1}^{N_{f}} \sum_{l=0}^{\left|q_{I}\right|-1} \Omega_{I}^{\Theta_{l}}=\frac{1}{2} \sum_{I=1}^{N_{f}} \operatorname{sgn}\left(m_{I}\right) .
$$

Note that the sum in (3.37) results from shuffling the sum over the holonomy saddle and the sum over charged matters.

$\mathbb{W} \mathbb{C P}$. One way to avoid Coulombic flat directions, responsible for the above fractional result, is to introduce a neutral scalar multiplet, say $P$, which imposes a symmetry breaking condition among the charged matters. Consider, for example,

$$
W=P K\left(\rho_{I}\right)+L\left(\rho_{I}\right),
$$

where $K$ and $L$ are functions of gauge-invariant "radial" variables $\rho_{I}=\left|\vec{X}_{I}\right|^{2} / 2$. One can see easily that, with generic enough $K$ and $L$, the classical solutions to the supersymmetry condition would be isolated points in the space spanned by $\left\{P ; \rho_{I}\right\}$. Each of such points will generate contribution of \pm 1 .

As such, let us consider a $\mathbb{W} \mathbb{C P}^{N_{f}-1}$ model, whose charge-dependent superpotential $W(\rho, P)$ is given by

$$
W=C P\left(\sum_{I=1}^{N_{f}} q_{I} \frac{\left|\vec{X}_{I}\right|^{2}}{2}-\xi\right)=C P\left(\sum_{I} q_{I} \rho_{I}-\xi\right),
$$

requires the change of variables for each $I=1, \cdots, N_{f}$,

$$
\mathbf{v} \equiv\left|q_{I}\right|\left|\vec{x}_{I}\right| \mathbf{u}, \quad \vec{z}_{I} \equiv c p q_{I} \vec{x}_{I}, \quad s \equiv c\left(\sum_{J} q_{J} \rho_{J}-\xi\right)
$$

$$
\text { and } \quad \vec{y}_{K} \equiv \sqrt{q_{K}^{2} c^{2} p^{2}+q_{K}^{2} \mathbf{u}^{2}} \cdot \vec{x}_{K} \quad(\text { for } K \neq I),
$$

which brings us to

$$
\Omega_{I}^{\Theta_{*}=0}=\frac{\operatorname{sgn}\left(q_{I} c\right)}{(2 \pi)^{N_{f}+2+1 / 2}\left|q_{I}\right|} \int d s d^{3} \mathbf{v} d^{2\left(N_{f}-1\right)} y d^{2} z_{I}\left[\frac{1}{\left|\vec{z}_{I}\right|}-\left(-\frac{1}{\left|\vec{z}_{I}\right|}\right)\right] e^{-\tilde{V}_{\text {bosonic }}^{\Theta_{*}=0}}
$$

with $s \in \operatorname{sgn}\left(c q_{I}\right)\left(+\infty,-\operatorname{sgn}\left(q_{I}\right)|c| \xi\right)$ coming from a half range of $p>0$; the integration over $p<0$ is reflected in the second term in the square bracket. As before, we have a quadratic exponent

$$
\tilde{V}_{\text {bosonic }}^{\Theta_{*}=0}=\frac{1}{2} \mathbf{v}^{2}+\frac{1}{2} s^{2}+\frac{1}{2} \vec{z}_{I}^{2}+\frac{1}{2} \sum_{K \neq I} \vec{y}_{K}^{2},
$$


and the integral is entirely Gaussian. Note how the integral is split into two parts separated by $\partial_{\rho_{I}} W=0$. This will be perhaps the most important lesson we learn here.

Therefore, the partial contribution to the index $\Omega_{I}$ at trivial saddle is,

$$
\Omega_{I}^{\Theta_{*}=0}=\left\{\begin{array}{ll}
-\frac{1}{\left|q_{I}\right|} & q_{I} \xi>0 \\
0 & q_{I} \xi<0
\end{array} .\right.
$$

Note how the sign $\operatorname{sgn}\left(c q_{I}\right)$ canceled out in the end. Collecting $\left|q_{I}\right|$ holonomy saddle contributions, we find

$$
\Omega=\sum_{I=1}^{N_{f}} \sum_{l=0}^{\left|q_{I}\right|-1} \Omega_{I}^{\Theta_{l}}=\left\{\begin{array}{ll}
-N_{f} & q_{I} \xi>0 \\
0 & q_{I} \xi<0
\end{array},\right.
$$

where we have assumed that all charges are of the same sign. This model has no asymptotic gapless directions, hence we find an integral index in the end.

Multiple charged scalars and no neutrals. Generalizing the previous example, let us turn to a theory of multiple charged scalars with a general radial superpotential $W=$ $W\left(\rho_{1}, \cdots, \rho_{N_{f}}\right)$. Recall the index is expressed by the sum of at most $\left(2 N_{f}+3\right)$-dimensional integrals (3.33). Each term is defined per charged matter at given holonomy saddle. Take a specific $\left(Q \vec{X}_{I}\right)$-led term $\Omega_{I}$ at the trivial saddle, for example,

$$
\Omega_{I}^{\Theta_{*}=0}=\frac{1}{(2 \pi)^{N_{f}+2}} \int d^{3} \mathbf{v} d^{2\left(N_{f}-1\right)} y d^{2} z_{I} \frac{\operatorname{sgn}\left(W_{I}\right)}{\left|q_{I}\right|\left|\vec{z}_{I}\right|} e^{-\tilde{V}_{\text {bosonic }}^{\Theta_{*}}}
$$

where the change of variables is noted by (3.29) for specific $I \in\left\{1, \cdots, N_{f}\right\}$.

An important point on the integration variables and the domains thereof should be addressed. Given how the superpotential is a function of the radial variables $\rho_{I}$ 's rather than individual $\vec{X}_{I}$, it is important to consider (3.45) as an integral over $\rho_{I}$. This means that the domain is really $\mathbb{R}_{+}^{N_{f}}$, which we will split as

$$
\mathbb{R}_{+}^{N_{f}}=\cup_{a} \Delta_{I}^{(a)},
$$

separated by the locus $0=W_{I} \equiv \partial_{\rho_{I}} W$, so that $W_{I}$ has a definite sign on each subdomain $\Delta_{I}^{(a)}$. With this, (3.45) can be more precisely written as

$$
\Omega_{I}^{\Theta_{*}=0}=\sum_{a} \frac{\operatorname{sgn}\left(W_{I}\left(\Delta_{I}^{(a)}\right)\right)}{(2 \pi)^{N_{f}+2}\left|q_{I}\right|} \int_{\mathbb{R}^{3}} d^{3} \mathbf{v} \int_{Y_{\Delta_{I}^{(a)}}} d^{2\left(N_{f}-1\right)} y d^{2} z_{I} \frac{1}{\left|\vec{z}_{I}\right|} e^{-\tilde{V}_{\text {bosonic }}}
$$

where $Y_{\Delta_{I}^{(a)}}$ is the image of $\Delta_{I}^{(a)}$ under the map (3.29). Let us introduce following variables parametrizing the image $Y_{\Delta_{I}^{(a)}}$,

$$
\lambda_{I}=\frac{1}{2} \vec{z}_{I}^{2}, \quad \sigma_{J(\neq I)}=\frac{1}{2} \vec{y}_{J}^{2},
$$

which span $\tilde{\mathbb{R}}_{+}^{N_{f}}$ at most. Our claim is that integral (3.47) vanishes for most of these domains, leaving behind a single unbounded domain for each $I$, to be denoted by $\Delta_{I}^{\infty}$ with the image $Y_{\Delta_{I}^{\infty}}$. 
Note that the boundary $\partial \Delta_{I}^{(a)}$ is composed of

$$
W_{I}=0, \quad \rho_{I}=0, \quad \rho_{J(\neq I)}=0,
$$

and the boundary at infinity. The points satisfying one of the first two equations are mapped to the $\lambda_{I}=0$ hyperplane by the reparameterization (3.29) while the third kind is mapped to the $\sigma_{J(\neq I)}=0$ hyperplane. This restricts $Y_{\partial \Delta_{I}^{(a)}}$, the image of boundary $\partial \Delta_{I}^{(a)}$, within $\lambda_{I}=0$ or within $\sigma_{J(\neq I)}=0$ hyperplane.

Figure 1 briefly depicts the splitting and mapping of the matter integration domain for $N_{f}=2$ theory, for $\Omega_{I=1}$. The green curve in the left quadrant is $W_{1}=0$. Note how, for exmple, all components of $\partial \Delta_{1}^{(1)}$, the bold colored lines, are all mapped to the two axes in the right figure. The dashed line on the right quadrant is the part of the boundary $\partial Y_{\Delta_{1}}^{(1)}$ that doesn't belong to the image of $\partial \Delta_{1}^{(1)}$. Here, the image of $\Delta_{1}^{(1)}$ folds itself, which means that the integral cancels out between the two sides. Such a folding could happen multiple times with the same result. This means

$$
\frac{\operatorname{sgn}\left(W_{1}\left(\Delta_{1}^{(1)}\right)\right)}{(2 \pi)^{N_{f}+2}\left|q_{I}\right|} \int_{\mathbb{R}^{3}} d^{3} \mathbf{v} \int_{Y_{\Delta_{1}^{(1)}}} d^{2\left(N_{f}-1\right)} y d^{2} z_{I} \frac{1}{\left|\vec{z}_{I}\right|} e^{-\tilde{V}_{\text {bosonic }}}=0 .
$$

The same mechanism works for most of the domains, bounded or unbounded. What matters is how the part of the boundary $\partial \Delta_{I}^{(a)}$ sitting at the locus $W_{I}=0$ is mapped to the axis $\lambda_{I}=0$. The other two possible boundaries are either $\rho_{I}=0$ or $\rho_{I}=\infty$. We will call $\Delta_{I}^{\infty}$ the domain that has the latter as part of the boundary. For all the other $\Delta_{I}^{(a)}$ 's, the boundary is mapped to $\lambda_{I}=0$ or $\sigma_{J \neq I}=0$ in such a way $Y_{\Delta_{I}^{(a)}}$ cancels itself out in the Gaussian integration (3.47) due to orientation flips. $\Delta_{I}^{\infty}$ is an exception to this, however, since one side of the boundary goes off to $\rho_{I} \rightarrow+\infty$, which is clearly not mapped to $\lambda_{I}=0$, so the cancelation of the integral need not happen. Rather, its image $Y_{\Delta_{I}^{\infty}}$ fully expands $\mathbb{R}_{+}^{N_{f}}$.

Hence, the surviving part of the integral is one over a single copy of $Y_{\Delta_{I}^{\infty}}$

$$
\frac{\operatorname{sgn}\left(W_{I}^{\infty}\right)}{(2 \pi)^{N_{f}+2}\left|q_{I}\right|} \int_{Y_{\Delta_{I}^{\infty}}} d^{2\left(N_{f}-1\right)} y d^{2} z_{I} \int_{\mathbb{R}^{3}} d^{3} \mathbf{v} \frac{1}{\left|\vec{z}_{I}\right|} e^{-\tilde{V}_{\text {bosonic }}},
$$

where $\operatorname{sgn}\left(W_{I}^{\infty}\right)$ is defined by the limit along specific direction

$$
\operatorname{sgn}\left(W_{I}^{\infty}\right) \equiv \operatorname{sgn}\left(W_{I}\left(\Delta_{I}^{\infty}\right)\right)=\lim _{\substack{\rho_{I} \rightarrow \infty \\ \rho_{J(\neq I)} \rightarrow 0}} \operatorname{sgn}\left(\frac{\partial W}{\partial \rho_{I}}\right) .
$$

Since the domain $Y_{\Delta_{I}^{\infty}}$ is always identical to $\tilde{\mathbb{R}}_{+}^{N_{f}}$, a theory with the radial superpotential always gives rise to the index of following form at the trivial holonomy saddle

$$
\Omega_{I}^{\Theta *}=0=\frac{1}{2} \frac{\operatorname{sgn}\left(W_{I}^{\infty}\right)}{\left|q_{I}\right|} .
$$

At each non-trivial holonomy saddle, a similar logic makes the computation simple: a bounded subdomain never contributes to the index, while a specific unbounded domain 

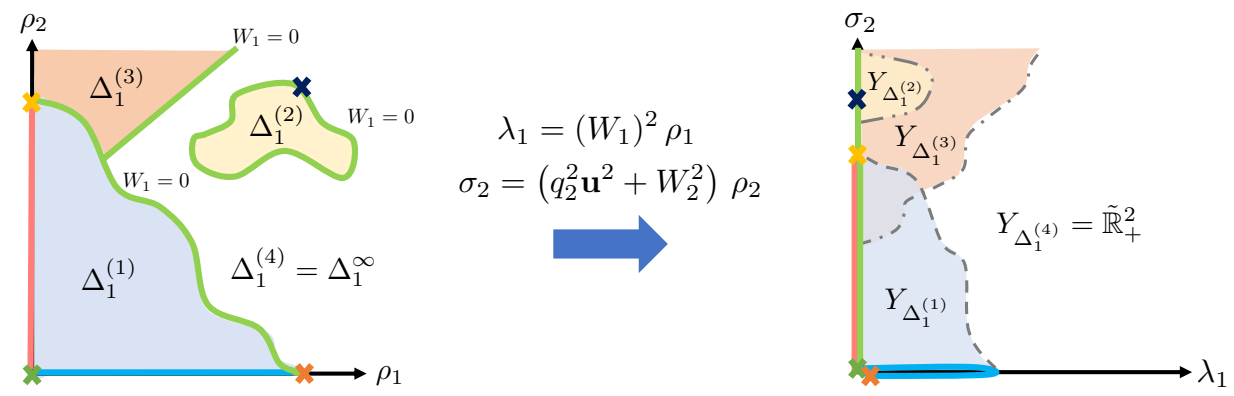

$Y_{\partial \Delta_{1}^{(1,2,3)}} \subset\left\{\lambda_{1}=0\right\} \times \mathbb{R}_{+}$

Figure 1. The domain decomposition and mapping under reparameterization (3.29) for $\Omega_{I=1}$ of the $N_{f}=2$ theory. The angular part is projected out to make the drawing simple. The image of boundary is different from the boundary of the image, i.e., $Y_{\partial \Delta_{I}^{(a)}} \neq \partial Y_{\Delta_{I}^{(a)}}$, generally, which underlies how most domains cancel out in the integral. $Y_{\Delta_{I=1}^{\infty}}=Y_{\Delta_{1}^{(4)}}$, whose projection to this $\left(\lambda_{1}, \sigma_{2}\right)$ plane equals the entire quadrant $\tilde{\mathbb{R}}_{+}^{2}$, is the only domain that can contribute to $\Omega_{I=1}^{\Theta_{*}=0}$.

generates a non-zero value, depending on the asymptotics of the superpotential. A straightforward holonomy saddle counting for charge- $q_{I}$ matters yields

$$
\Omega=\sum_{I=1}^{N_{f}} \sum_{l=0}^{\left|q_{l}\right|-1} \Omega_{I}^{\Theta_{l}}=\frac{1}{2} \sum_{I} \operatorname{sgn}\left(W_{I}^{\infty}\right),
$$

for generic radial $W$ without asymptotic flat directions. This completes the proof.

If we further assume that $W=c_{W} \cdot G\left(\rho_{1}, \cdots, \rho_{N_{f}}\right)$ where

$$
\partial_{I} G(\rho) \rightarrow+\infty
$$

for each $I$, along all asymptotic directions in the $\rho$ space, we have a simplified version of (3.54). Again $G$ is an analytic function with no singular behavior, especially at the origin. For instance this is guaranteed if $G$ is a polynomial with top degrees forming a non-degenerate homogenous polynomial with all positive coefficients since $\rho_{I}$ 's all live in the positive half lines. Then (3.54) is translated into

$$
\Omega=\frac{N_{f}}{2} \operatorname{sgn}\left(c_{W}\right) .
$$

A simple example of this is the $N_{f}=2$ Abelian gauge theory with quartic superpotential $W$,

$$
W\left(\rho_{1}, \rho_{2}\right)=\frac{c_{W}}{4}\left(\rho_{1}+\rho_{2}-v\right)^{2} .
$$

Given a positive real constant $v$, the integration domain for $\Omega_{1}$ can be split as follows,

$$
\Delta_{1}^{(1)}=\left\{\left(\rho_{1}, \rho_{2}\right) \mid \rho_{1}+\rho_{2}-v<0\right\}, \quad \Delta_{1}^{\infty}=\left\{\left(\rho_{1}, \rho_{2}\right) \mid \rho_{1}+\rho_{2}-v>0\right\} .
$$

In a similar way, the domain for the $\Omega_{2}$ integral is split as follows,

$$
\Delta_{2}^{(1)}=\left\{\left(\rho_{1}, \rho_{2}\right) \mid \rho_{1}+\rho_{2}-v<0\right\}, \quad \Delta_{2}^{\infty}=\left\{\left(\rho_{1}, \rho_{2}\right) \mid \rho_{1}+\rho_{2}-v>0\right\} .
$$


The exchange symmetry $\rho_{1} \leftrightarrow \rho_{2}$ in (3.57) makes the domain split in the same way for $\Omega_{1}$ and for $\Omega_{2}$.

Following the above argument, it turns out that $\Omega_{I \in\{1,2\}}$ integrals over $\Delta_{\{I=1,2\}}^{(1)}$ vanish. On the other hand, the integration over the unbounded domain converges to $\frac{1}{2} \operatorname{sgn}\left(c_{W}\right) /\left|q_{I}\right|$ since each domain covers $\mathbb{R}_{+}^{2} \times \mathbb{R}^{3}$ in $\left\{\lambda_{1}, \sigma_{2}, \vec{v}\right\}$ and $\left\{\sigma_{1}, \lambda_{2}, \vec{v}\right\}$ parametrizations, respectively. Thus

$$
\Omega_{I}^{\Theta_{*}=0}=\frac{1}{2\left|q_{I}\right|} \cdot \operatorname{sgn}\left(c_{W}\right)
$$

at the saddle at the origin. Computation at other saddles would be isomorphic to this; the only difference would be that the set of charged matters which enter the computation is selected by $\Theta_{*}$ and is in general a subset of those at $\Theta_{*}=0$. Summing over $\left|q_{I}\right|$ holonomy saddle contributions for each $I$, we have the index of (3.57)

$$
\Omega=\left(\frac{\left|q_{1}\right|}{2\left|q_{1}\right|}+\frac{\left|q_{2}\right|}{2\left|q_{2}\right|}\right) \operatorname{sgn}\left(c_{W}\right)=\operatorname{sgn}\left(c_{W}\right)
$$

consistent with (3.56).

\subsection{Why is Coulombic wall-crossing absent?}

Let us close the section with an observation. Consider the following three prototypes of theories we discussed above,

$$
W_{1}=\frac{1}{2} m|\vec{X}|^{2}, \quad W_{2}=P\left(\frac{1}{2}|\vec{X}|^{2}-\xi\right), \quad W_{3}=\frac{1}{2}\left(|\vec{X}|^{2}-\xi\right)^{2},
$$

with neutral and real $P$ and a unit-charged two-component field $\vec{X}$. The direct computation of the indices produced,

$$
\Omega_{1}=\left\{\begin{array}{rr}
1 / 2 & m>0 \\
-1 / 2 & m<0
\end{array}, \quad \Omega_{2}=\left\{\begin{array}{rr}
-1 & \xi>0 \\
0 & \xi<0
\end{array}, \quad \Omega_{3}=\frac{1}{2},\right.\right.
$$

so that the former two experiences wall-crossing while the last does not.

At $\xi=0$ all three theories admit gapless flat directions, a necessary condition for wall-crossing, so what is it that makes the difference? At $m=0$ or $\xi=0$, the classical vacuum manifolds are, respectively,

$$
\{\mathbf{u}\} \cup\{\vec{X}\}, \quad\{\mathbf{u}\} \cup\{P\}, \quad\{\mathbf{u}\},
$$

so one immediate difference is the presence/absence of the flat direction associated with the matter field. In the last theory with no wall-crossing, at most the Coulombic direction become asymptotically flat. Is there a way to understand this phenomenon in general term? It turns out that there is a simple explanation, not from $d=1$ physics but rather from the possibility of embedding such theories as the lowest Kaluza-Klein sector of a $d=3$ Chern-Simons theory. 
Consider the above theories as coming from $\mathbb{T}^{2}$ compactification of $d=3 \mathcal{N}=1$ gauge theories. One would compute the Witten index of such a theory by performing the path integral on $\mathbb{T}^{3}$. Since the size of the torus cannot enter the twisted partition function of the gapless theory, one could imagine sending the spatial radius to zero first and thus reduce the path integral to that of a $d=1 \mathcal{N}=2$ theory. If we were considering $d=3$ theories, therefore, the Coulombic direction is no longer asymptotic as the bosonic variables live in a dual torus, hence cannot produce asymptotic flat directions. Is this why there is no wall-crossing in $d=1$ as well when the asymptotic direction is entirely Coulombic?

Actually this is too rash. Consider the well-known wall-crossing story with the twice supersymmetries, where the $d=1 \mathcal{N}=2$ vector multiplet is accompanied by one more real adjoint scalar multiplet, call it $\Sigma$. It is well-known that this system generically wallcrosses due to wavefunctions that leak out to the asymptotic Coulomb phase including the $\Sigma$ directions. The Coulombic physics is notoriously dimension-dependent, which actually underlies why $\mathcal{N}=4$ quantum mechanics wall-crosses while $d=2 \mathcal{N}=(2,2)$ GLSM does not. As such the above naive argument that u's originate from periodic variables does not immediately preclude wall-crossing due to the Coulombic asymptotic infinity in strict $d=1$ theories.

We believe that the difference comes from how the Chern-Simons terms, when a $d=1$ gauge theory is lifted to $d=3$, control infrared issues on the Coulombic side. If one can embed a given $d=1 \mathcal{N}=2$ gauge theory to a $d=3$ Chern-Simons theory with arbitrary nonzero level $\kappa$, the latter gaps out $d=1 \mathcal{N}=2$ Coulombic physics by turning it into a Landau problem, as we will see in next section. Even in the lowest Landau level, infinitely degenerate in the infinitesimal $\mathbb{T}^{2}$ limit, individual states are enumerative and of fixed size controlled by the effective magnetic field and angular momentum in the $u_{1,2}$ plane. The runaway behavior of ground states necessary for wall-crossing is impossible, and this makes wall-crossing due to the Coulombic flat directions impossible.

One might wonder if this explanation makes any sense since $d=1$ limit of the ChernSimons level is subtle. With any finite $\kappa$, one ends up with a Landau problem with infinite total flux when a strict $d=1$ limit is taken. Thus we are effectively studying a dimensional reduction of $\kappa=0$ theory. However, as we will see in the next section, $\kappa$-independent part of the $d=3$ twisted partition functions, which we will call $\Omega_{0}$, share the common building blocks with $d=1 \Omega$. These are nothing but $\Omega_{I}^{\Theta_{*}=0}$ 's above, and the difference between $d=3 \Omega_{0}$ and $d=1 \Omega$ is merely in the details of the holonomy saddles.

This means that $d=1$ wall-crossing behavior, which would be entirely due to discontinuity of $\Omega_{I}^{\Theta_{*}^{*}=0}$, is connected to $d=3$ wall-crossing of this $\kappa$-independent $\Omega_{0}$. The latter's insensitivity to $\kappa$ tells us, on the other hand, that nothing special happens at $\kappa=0$ with $\Omega_{0}$, and thus with $\Omega_{I}^{\Theta_{*}=0}$. Therefore, the absence of the $d=1$ wall-crossing from the Coulombic continuum can be argued with a mere logical possibility of $d=3$ Chern-Simons lift, even though we are effectively studying dimensional reduction of the $\kappa=0$ version.

With such a Chern-Simons uplift, the wall-crossing that does happen in $d=1 \mathcal{N}=4$ will occur due to the asymptotics of $\Sigma$, the real adjoint matter that enlarges the vector multiplet to the one with the twice supersymmetries. In a more conventional localization computation, where one introduces an $R$-charge chemical potential as an infrared regulator, 
again it is the $\Sigma$ direction that is left unaffected and responsible for the wall-crossing. In our $d=1 \mathcal{N}=2$ viewpoint, however, $\Sigma$ is one of real scalar multiplets. We conclude that in $d=1 \mathcal{N}=2$ (gauge) theories, a wall-crossing occurs only if there are asymptotic gapless directions that emerge along the scalar multiplet side.

\section{$4 d=3 \mathcal{N}=1$ Chern-Simons-Matter}

In this final section, we will explore $d=3$ version of the same problem for $\mathrm{SO}(2)$ ChernSimons-Matter theories. Witten indices of $d=3$ massive theories can be computed by compactification on $\mathbb{T}^{2}$, which would bring us to an extension of $d=1$ computations, which we wish to exploit here.

\section{1 $d=3 \mathcal{N}=2$ Chern-Simons-Matter revisited}

Before we delve into $d=3 \mathcal{N}=1$ theories, it is worthwhile to recall how vacuum countings work for $d=3 \mathcal{N}=2$ theories [26]. For massive $d=3$ theories, the quantum vacuum counting at a symmetric phase can be most easily done by integrating out the matter fields first. For such contributions, one can resort to the previous countings by Witten of pure Chern-Simons theories [27]. When vacua with the gauge symmetry broken are present, one must also consider them, so generally we have

$$
\mathcal{I}=\mathcal{I}_{\text {symmetric }}+\mathcal{I}_{\text {broken }}
$$

For $d=3 \mathcal{N}=2$ theories with real masses, Intriligator and Seiberg gave a beautiful demonstration [24] of how to count these vacua and to classify them, and how the sum remains robust although individual contribution might vary with parameters of the theory.

Let us take a simple $\mathcal{N}=2$ theory, say, an $\mathrm{SO}(2)_{\kappa}$ theory with a chiral multiplet $\Phi=\left(X_{1}+i X_{2}\right) / \sqrt{2}$ of charge $q>0$ for an illustration. The bosonic potential takes the form

$$
\mathcal{V}_{\mathcal{N}=2}=\frac{1}{2}\left(q|\vec{X}|^{2}-\xi-\kappa \Sigma\right)^{2}+2 q^{2} \Sigma^{2}|X|^{2}
$$

modulo the electric coupling $e^{2}$, where the would-be $\mathcal{N}=1$ scalar multiplet $\Sigma$ now belongs to the $\mathcal{N}=2$ vector multiplet. Let us assume $|\kappa|>q^{2} / 2$, just to avoid the clutter coming from one-loop shift of $\kappa$. The potential admits two types of vacua,

$$
\left\{\Sigma=0, q|\vec{X}|^{2}=\xi\right\}, \quad\left\{\Sigma=-\xi / \kappa,|\vec{X}|^{2}=0\right\},
$$

of which the former exists only if $\xi>0$. The existence of a symmetric vacuum is a universal feature of $\mathcal{N}=2$ Chern-Simons theories for large enough $|\kappa|$, which will not be the case for $d=3 \mathcal{N}=1$ theories.

Here we will follow a reasoning which is similar to [24], but in an $\mathcal{N}=1$ theory language, with a care given to the sign convention for the Witten index. In our $\mathcal{N}=1$ notation, the would-be $D$-term potential above arises from the superpotential

$$
W=\Sigma\left(q|\vec{X}|^{2}-\xi-\frac{1}{2} \kappa \Sigma\right)
$$


Note that the singlet $\Sigma$ comes with mass $-\kappa$. Let us expand this in the two phases as

$$
\begin{gathered}
\delta W_{\text {broken }}(\Sigma, \delta|\vec{X}|) \simeq+\sqrt{q \xi} \Sigma \delta|\vec{X}|-\frac{1}{2} \kappa \Sigma^{2} \\
\delta W_{\text {symmetric }}(\delta \Sigma, \vec{X}) \simeq-(q \xi / \kappa)|\vec{X}|^{2}-\frac{\kappa}{2}(\delta \Sigma)^{2} .
\end{gathered}
$$

For instance, the broken vacuum for $\xi>0$ will contribute

$$
\mathcal{I}_{\text {broken }}=\left\{\begin{array}{cc}
-q^{2} & \xi>0 \\
0 & \xi<0
\end{array}\right.
$$

The $q^{2}$ degeneracy can be understood from $q^{2}$ many discrete gauge holonomies along spatial $\mathbb{T}^{2}$, remnants from the partial symmetry-breaking $\mathrm{SO}(2) \rightarrow \mathbb{Z}_{q}$. Note that this is one more manifestation of the holonomy saddle, although now in the context of the canonical approach, as opposed to the path integral approach. On the other hand, the minus sign arises from the determinant of the $2 \times 2$ mass matrix in $\delta W_{\text {broken }}$; the contribution from these two gauge singlet real fields $\Sigma$ and $\delta|\vec{X}|$ can be found by compactification on $\mathbb{T}^{3}$ and thus by our $d=1$ computation easily.

In the symmetric phase, contributions from $\vec{X}$ and $\delta \Sigma$ play different roles. $\vec{X}$ can be first integrated out and shift the Chern-Simons level to

$$
\kappa_{\mathrm{eff}}=\kappa+\frac{1}{2} \operatorname{sgn}(-\xi / \kappa) q^{2} .
$$

On the other hand, $\delta \Sigma$ factorizes out from the Chern-Simons dynamics and merely affects the overall sign of the index such that the contribution to the index from the symmetric vacuum is

$$
\mathcal{I}_{\text {symmetric }}=\operatorname{sgn}(-\kappa) \kappa_{\text {eff }}=-|\kappa|+\frac{1}{2} \operatorname{sgn}(\xi) q^{2},
$$

where we invoked the well-known index, $\kappa$, for the pure $\mathrm{SO}(2)_{\kappa}$ theory. Therefore, for either sign of $\xi$, we find

$$
\mathcal{I}=\mathcal{I}_{\text {symmetric }}+\mathcal{I}_{\text {broken }}=-|\kappa|-\frac{1}{2} q^{2},
$$

and that there is no wall-crossing at $\xi=0$, consistent with the absence of any asymptotic flat direction appearing.

One thing we wish to note here is that all of the above was possible only thanks to the very rigid $D$-term potential, although we recast it into the $\mathcal{N}=1$ superpotential language. So, what could be done if we really consider general $d=3 \mathcal{N}=1$ theories with a generic and not-so-simple superpotential $W$ ? Keeping track of the symmetric and the broken vacua may not be so feasible in such general theories. Recall that the Witten index in section 2 only requires a winding number information of $W$ and has little to do with details at finite $X$. If we wish to find an analog of the above counting there, if any, it would be the Morse theory interpretation. On the other hand, we are not aware of how to extend the Morse theory to the gauged dynamics. 
What should be done is of course to go back to the $d=3 \mathcal{N}=1$ path integral and ask whether there is a way to reduce it to ordinary Gaussian integrals and eventually to the winding number information in $W$, on par with what we have seen for $d=1 \mathcal{N}=2$ theories in section 2. The rest of this note is devoted to this question.

\section{2 $d=1$ approach for $d=3$ Chern-Simons-Matter}

Suppose we want to compute the same Witten index such as (4.9) for general $d=3 \mathcal{N}=1$ Chern-Simons theories, entirely by compactifying the theory on $\mathbb{T}^{3}$ and performing the Euclidean path integral. This will bring us to the kind of computation in the two previous sections. The Kaluza-Klein sector cancels out neatly in the end, so the reduction to $d=1$ is achieved naturally. The new ingredient is the remnant of the Chern-Simons term in the $d=1$ Lagrangian,

$$
\sim-\frac{\kappa L_{1} L_{2}}{2 \pi} u_{1} \partial_{t} u_{2}
$$

as well as its accompanying mass term for gaugino $\lambda$. The holonomies $u_{a}$ 's are of $2 \pi / L_{a}$ periods, where $L_{1,2}$ are the lengths of the two spatial circles in $\mathbb{T}^{3}$.

This means that the kinetic part of the vector multiplet is the same as that of the Landau problem with the magnetic field $\kappa L_{1} L_{2} / 2 \pi$ and the total flux $\Phi_{B}=2 \pi \kappa$. Note that since we will compute this on arbitrarily small torus, $\kappa$ here is the bare Chern-Simon level. Since the magnetic field couples to $u_{1,2}$ at quadratic level with a single time derivative, the heat kernel expansion should be in principle worked out from scratch. In appendix A, we derive the 0 -th order heat kernel for the Landau problem, necessary for the heat kernel expansion. As observed there, the small $\beta$ expansion, combined with the insertion of $(-1)^{F}$, is such that the computation we have performed so far in the absence of the external magnetic field is applicable. The only change in the end is the extra fermion bilinear $\sim\left(\kappa L_{1} L_{2}\right) \lambda \lambda$ which affects the Pfaffian part of the computation.

The path integral has a somewhat different decomposition than the counting based on $d=3$ vacuum analysis above. For rank 1 theories, we find

$$
\Omega^{3 d}=\Omega_{0}+\mathcal{Z}_{\kappa} \int \frac{d u_{1} d u_{2} d u_{3}}{(2 \pi)^{3}} \beta L_{1} L_{2} \kappa=\Omega_{0}+\kappa \cdot \mathcal{Z}_{\kappa},
$$

where $\Omega_{0}$ is part of the path integral independent of $\kappa$. Here, we displayed variables prior to the $\beta$-scaling, so that $u_{3}$ is the holonomy along the time direction of period $2 \pi / \beta$, and similarly $u_{1,2}$ have the periods $2 \pi / L_{1,2}$. For higher rank theories, we similarly expect higher order pieces in $\kappa$ 's to show up as well, although here we confine ourselves to $\mathrm{SO}(2)$ theories.

The second term in (4.11) can be understood, from the $d=1$ perspective, easily since the supersymmetric Landau level problem comes with the ground state degeneracy of $\left|\Phi_{B}\right| / 2 \pi=|\kappa|$, which equals the usual level-by-level degeneracy for the purely bosonic Landau problem. For the lowest Landau level, the $\mathrm{SO}(2)$ gaugino merely shifts the ground state energy to zero and does not affect this degeneracy. $\mathcal{Z}_{\kappa}$ takes into a further degeneracy factor, with sign, that, for example, might come from a decoupled neutral sector, tensored with this Landau problem. 
As far as such a path integral on $\mathbb{T}^{3}$ goes, $\Omega_{0}$ and $\mathcal{Z}_{\kappa}$ are computed blindly, regardless of the classical vacuum structure. This means that the $d=3$ index has two different decompositions,

$$
\mathcal{I}=\mathcal{I}_{\text {symmetric }}+\mathcal{I}_{\text {broken }}=\kappa \cdot \mathcal{Z}_{\kappa}+\Omega_{0} .
$$

While $\mathcal{I}_{\text {symmetric }}$ includes the $\kappa$-dependent pieces, it also has the additive one-loop shift of $\kappa$ from charged matters, so the two decompositions are not the same in the presence of charged matters. $\Omega_{0}$ can and will generally contribute to both $\mathcal{I}_{\text {symmetric }}$ and $\mathcal{I}_{\text {broken }}$.

For $d=3 \mathcal{N}=1$ theories, the classical vacua are determined by the more flexible superpotential $W$, instead of the rigid $D$-term potential (4.2) above. Also, unlike $\mathcal{N}=2$ theories, where the $D$-term potential with large enough $|\kappa|$ always admits a symmetric vacuum, one can easily imagine $W$ that does not admit any symmetric vacuum even for large enough $|\kappa|$. Since, from $d=3$ physics, it is clear that the $\kappa$-dependent part can get a contribution only from a symmetric vacuum, we find immediately that

$$
\mathcal{Z}_{\kappa}=0
$$

in theories with no classical symmetric vacuum. When an asymptotically flat direction is present, one may need to understand this as

$$
\lim \mathcal{Z}_{\kappa}=0,
$$

where the limit is taken to scale up the finite asymptotic gap, as usual. On the other hand, if the theory admits exactly one symmetric vacuum classically, we should expect $\left|\mathcal{Z}_{\kappa}\right|$, or the above limit thereof if needed, to equal 1.

Such relatively simple behaviors of $\mathcal{Z}_{\kappa}$ will manifest in the path integral computation as well; we will see presently that this quantity is really determined by the neutral sector of the matter content. In particular, we will see that, for $\mathcal{N}=1 \mathrm{SO}(2)$ theories with no neutral scalar multiplets, $\mathcal{Z}_{\kappa}=1$ has to hold universally for in our sign convention. By the same token, we end up finding $\mathcal{Z}_{\kappa}= \pm 1,0$ at most, with the above limit taken if necessary and with wall-crossing possibilities, if a single neutral scalar multiplet is present.

Reduction of the path integral. Let us now turn to the evaluation of

$$
\Omega^{3 d}=\kappa \cdot \mathcal{Z}_{\kappa}+\Omega_{0},
$$

for a general superpotential. The two terms $\Omega_{0}$ and $\mathcal{Z}_{\kappa}$ are computed from the two additive pieces in the Pfaffian of the fermion bilinear, which for the current Abelian problem has the simple form,

$$
M(X, u)=\left(\begin{array}{cc}
-\frac{\kappa L_{1} L_{2}}{2 \pi} \epsilon_{a b} & (Q X)_{\nu} \epsilon_{a c} \\
\left(X Q^{\dagger}\right)_{\mu} \epsilon_{d b} & \partial_{\mu} \partial_{\nu} W \epsilon_{d c}+u_{i} Q_{\mu \nu}\left(\epsilon \cdot \sigma^{i}\right)_{d c}
\end{array}\right)
$$

$\Omega_{0}$ results from the Pfaffian of this matrix with $\kappa L_{1} L_{2} \rightarrow 0$ while $\mathcal{Z}_{\kappa}$ is from the piece linear in the Chern-Simons mass term on the upper-left corner. Therefore, the matrix relevant for $\mathcal{Z}_{\kappa}$ is much simpler,

$$
\left(M_{\kappa}\right)_{\mu \nu} \equiv \partial_{\mu} \partial_{\nu} W \epsilon_{d c}+u_{i} Q_{\mu \nu}\left(\epsilon \cdot \sigma^{i}\right)_{d c}
$$


which is the mass matrix for the matter fermions only; the $\kappa$-linear piece saturated the gaugino zero modes. A useful decomposition of the entire Pfaffian is

$$
\operatorname{Pf}(\tilde{M})=-\frac{\tilde{\kappa}}{2 \pi} \cdot \operatorname{Pf}\left(\tilde{M}_{\kappa}\right)+\sum_{I}\left(Q \vec{X}_{I}\right) \cdot \operatorname{Pf}\left(\tilde{M}_{I}\right),
$$

where the latter sum leads to $\Omega_{0}$ being expressed as a sum over the flavor index $I=$ $1, \cdots, N_{f}$. We refer the readers to section 3.3 for the complete details on the latter sum.

In the end, we find that both $\Omega_{0}$ and $\mathcal{Z}_{\kappa}$ are expressed as simple and factorized Gaussian integrals with all the nontrivial information encoded in the integration domain. In other words, we find an analog of (2.32) for the $d=3$ Chern-Simons gauge theory. One important point we need to emphasize is that, for $d=3$ theories, $\kappa$ which enters our path integral approach is the UV one, as various examples below will show. This is natural since we are evaluating the index in the small torus $\mathbb{T}^{3}$ limit, which effectively cuts off the infrared end of the path integral.

Gaussian reduction for $\mathcal{Z}_{\kappa}$. The simpler form of $M_{\kappa}$ suggests a further simplification for $\mathcal{Z}_{\kappa}$. As we show in appendix B, u's are decoupled completely from the rest, for the computation of $\mathcal{Z}_{\kappa}$, upon the following universal change of variables,

$$
\mathbf{v}=\mathbf{u}, \quad s_{A}=\partial_{p_{A}} W, \quad \vec{y}_{I}=\sqrt{q_{I}^{2} \mathbf{u}^{2}+\left(\partial_{\rho_{I}} W\right)^{2}} \cdot \vec{x}_{I},
$$

for $\beta$-scaled two-component charged matter $\vec{x}_{I}$ of charge $q_{I}$ and neutral real scalar $p_{A}$, while the gauge holonomies $\mathbf{u}$ are left intact. This results in a clean decoupling of $\mathbf{u}$ from the matter integral, leading to the factorized form of the second term in (4.11). Note that this change of variables is the same one as in section 3.2 where the Witten index $\Omega$ of $d=1$ massive gauge theory is computed.

The Jacobian to (4.19) turns out to be equal precisely to minus the Pfaffian of matteronly minor $\tilde{M}_{\kappa}$,

$$
\operatorname{Pf}\left(\tilde{M}_{\kappa}\right)=-\operatorname{Det}\left(\frac{\partial\left(\vec{y}_{I}, \mathbf{v}\right)}{\partial\left(\vec{x}_{I}, \mathbf{u}\right)}\right)
$$

the proof of which can be found in appendix B. Simultaneously, the bosonic potential is written in terms of new variables (4.19)

$$
\tilde{V}_{\text {bosonic }}=\frac{1}{2} \sum_{A=1}^{\operatorname{dim}(S)} s_{A}^{2}+\frac{1}{2} \sum_{I=1}^{N_{f}} \vec{y}_{I}^{2} .
$$

The bosonic potential $\tilde{V}$ is now independent of new three-dimensional holonomy variable v. This leads to

$$
\begin{aligned}
\mathcal{Z}_{\kappa} & =\frac{1}{(2 \pi)^{N_{f}+\frac{\operatorname{dim}(S)}{2}}} \int d^{2 N_{f}} y d^{\operatorname{dim}(S)} s \exp \left(-\frac{1}{2} \sum_{A} s_{A}^{2}-\frac{1}{2} \sum_{I} \vec{y}_{I}^{2}\right) \\
& =\frac{1}{(2 \pi)^{\frac{\operatorname{dim}(S)}{2}}} \int d^{\operatorname{dim}(S)} s \int \prod_{I} d\left(\vec{y}_{I}^{2} / 2\right) \exp \left(-\frac{1}{2} \sum_{A} s_{A}^{2}-\sum_{I}\left(\vec{y}_{I}^{2} / 2\right)\right)
\end{aligned}
$$


because the integration domains of $\vec{y}_{I}$ and $s_{A}$ are also independent of $\mathbf{v}$ in the end. Please note that, for $\mathcal{Z}_{\kappa}$, the holonomy saddle is irrelevant. No saddle appears because the integral is free along the $\mathbf{v}$ directions and the contribution from the holonomy torus enters via its volume, as in (4.11).

In fact, this Gaussian integration for $\mathcal{Z}_{\kappa}$ can be universally reduced to a reduced theory with neutral scalar multiplets only on par with (2.32). If no neutral scalars are present, on the other hand, in particular, we will see that $\mathcal{Z}_{\kappa}=1$ universally. See the next subsection for a derivation of this.

Gaussian reduction for $\Omega_{I}^{\Theta_{*}=0}$. For $\Omega_{I}^{\Theta_{*}}$ with a general superpotential, the computation proceeds exactly in the same way as for those that entered $d=1 \Omega$ in section 3.3. Here we merely reiterate results there for the sake of completeness, and shortly turn to $\Omega_{0}$ which must be built up from $\Omega_{I}^{\Theta_{*}}$ with the holonomy saddles taken into account. We will come back to this in the last part of this subsection.

For $\Omega_{I}^{\Theta_{*}=0}$, we must resort to the alternate coordinate systems, introduced in section 3.3. These can be summarized as $N_{f}$ many sets of distinct coordinates, labeled by $I$,

$$
\begin{aligned}
\mathbf{v} & =\left|q_{I}\right|\left|\vec{x}_{I}\right| \mathbf{u}, & s_{A} & =\partial_{p_{A}} W, \\
\vec{z}_{I} & =\left(\partial_{\rho_{I}} W\right) \vec{x}_{I}, & \vec{y}_{K(\neq I)} & =\sqrt{q_{K}^{2} \mathbf{u}^{2}+\left(\partial_{\rho_{K}} W\right)^{2}} \cdot \vec{x}_{K},
\end{aligned}
$$

to be used upon decomposing the Pfaffian for $\Omega_{0}$ into $N_{f}$ many summands. As was mentioned previously, the assumption $W=W\left(\rho_{I}, P_{A}\right)$ can be justified given $\mathrm{SO}(2)^{N_{f}-1}$ flavor symmetries, which we believe to be innocuous for the purpose of computing the index for massive theories.

For each doublet label $I \in\left\{1, \cdots, N_{f}\right\}$, the same set of integration variables is suggested as in (3.29), so that its Jacobian simplifies the $\tilde{\kappa}$-independent piece of Pfaffian, denoted by $\left(Q \vec{X}_{I}\right) \cdot \operatorname{Pf}\left(\tilde{M}_{I}\right)$, as follows:

$$
\left(Q \vec{X}_{I}\right) \cdot \operatorname{Pf}\left(\tilde{M}_{I}\right)\left|\frac{\partial(\vec{x}, p, \mathbf{u})}{\partial(\vec{z}, \vec{y}, s, \mathbf{v})}\right|=\frac{1}{\left|q_{I}\right| W_{I}\left|\vec{x}_{I}\right|}=\frac{1}{\left|q_{I}\right| \operatorname{sgn}\left(W_{I}\right)\left|\vec{z}_{I}\right|} .
$$

We are yet to find a general analytical proof of this but have checked its veracity for all examples in this note up to $N_{f}=3$.

Also, unlike $\mathcal{Z}_{\kappa}$, the holonomy saddles become relevant and contribute additively to $\Omega_{0}$, the $\kappa$-independent piece of the partition function. For instance, the $I$-th contribution of the saddle at the origin $\Omega_{I}^{\Theta_{*}=0}$ reads,

$$
\begin{aligned}
\Omega_{I}^{\Theta_{*}=0} & =\frac{1}{(2 \pi)^{N_{f}+2+\frac{\operatorname{dim}(S)}{2}}} \int d^{3} \mathbf{v} d^{2\left(N_{f}-1\right)} y d^{2} z_{I} d^{\operatorname{dim}(S)} s \frac{1}{\left|q_{I}\right| \operatorname{sgn}\left(W_{I}\right)\left|\vec{z}_{I}\right|} e^{-\tilde{V}_{\text {bosonic }}} \\
& =\frac{1}{(2 \pi)^{2+\frac{\operatorname{dim}(S)}{2}}} \int d^{3} \mathbf{v} d^{\operatorname{dim}(S)} s \int d\left(\vec{z}_{I}^{2} / 2\right) \prod_{J \neq I} d\left(\vec{y}_{J}^{2} / 2\right) \frac{1}{\left|q_{I}\right| \operatorname{sgn}\left(W_{I}\right)\left|\vec{z}_{I}\right|} e^{-\tilde{V}_{\text {bosonic }}},
\end{aligned}
$$

with

$$
\tilde{V}_{\text {bosonic }}=\frac{1}{2} \mathbf{v}^{2}+\frac{1}{2} \sum_{A=1}^{\operatorname{dim}(S)} s_{A}^{2}+\frac{1}{2} \vec{z}_{I}^{2}+\frac{1}{2} \sum_{J \neq I} \vec{y}_{J}^{2},
$$


where again everything reduces to a Gaussian integral and all the nontrivial information of the theory is transferred to the integration domain, on par with (2.32).

Building up $\boldsymbol{\Omega}_{\mathbf{0}}$ from holonomy saddles on $\mathbb{T}^{3}$. If there were no holonomy saddle except at $\Theta_{*}=0$, i.e. if $\left|q_{I}\right|=1$ for all $I$, we would have

$$
\Omega_{0}=\sum_{I} \Omega_{I}^{\Theta *=0}
$$

More generally, however, we need to take account of holonomy saddles. We follow the same logic of section 3.2 by taking into account the additional holonomy saddles due to the spatial $\mathbb{T}^{2}$ as well. For a $d=3$ theory, the holonomy saddles are labeled by three angles on the dual torus of $\mathbb{T}^{3}: \Theta_{*}=\Theta_{l, m, n}=\left(\theta_{l}, \theta_{m}, \theta_{n}\right)$. One can have an additive $\Omega_{K}^{\Theta_{*}}$ contribution only if

$$
\theta_{l} q_{K} \in 2 \pi \mathbb{Z}, \quad \theta_{m} q_{K} \in 2 \pi \mathbb{Z}, \quad \theta_{n} q_{K} \in 2 \pi \mathbb{Z},
$$

for all three holonomies, determining the charged scalar multiplets $\vec{X}_{K}$ that enter the path integral contribution at $\Theta_{*}$.

Let us denote the collection of such $K$ 's, $\mathcal{N}_{*}$ or $\mathcal{N}_{l, m, n}$ to be precise. We then proceed in the same way for the reduced theory sitting at $\Theta_{*}$, and find additive contributions,

$$
\sum_{K \in \mathcal{N}_{*}} \Omega_{K}^{\Theta_{*}}
$$

where the superscript $\Theta_{*}$ indicates the low energy theory at the holonomy saddle, obtained by removing all charged scalar multiplets such that $q_{J} \theta_{i} / 2 \pi$ is non-integral at least for one of $i=l, m, n$. In fact, a necessary condition for $\Theta_{*}$ to be a contributing holonomy saddle for Abelian theories is that $\mathcal{N}_{*}$ is not empty. As such, we have the general routine,

$$
\Omega_{0}=\sum_{\Theta_{*}} \sum_{K \in \mathcal{N}_{*}} \Omega_{K}^{\Theta_{*}}=\sum_{l, m, n} \sum_{I} \Omega_{I}^{\Theta_{l, m, n}} \delta_{l, m, n}\left(q_{I}\right),
$$

where the formal presence of the ill-defined $\Omega_{I}^{\Theta_{l, m, n}}$ for $I \notin \mathcal{N}_{l, m, n}$ is immaterial since

$$
\delta_{l, m, n}\left(q_{I}\right)=\delta_{\frac{q_{I} \theta_{l}}{2 \pi}} \delta_{\frac{q_{I} \theta_{m}}{2 \pi}} \delta_{\frac{q_{I} \theta_{n}}{2 \pi}}
$$

is designed to vanish for such $I$ 's, and is 1 if $I=K \in \mathcal{N}_{l, m, n}$

This symbol also allows us to exchange the two summations as

$$
\Omega_{0}=\sum_{I} \sum_{l, m, n} \Omega_{I}^{\Theta_{l, m, n}} \delta_{l, m, n}\left(q_{I}\right)
$$

For $\mathrm{SO}(2)$ theories, there is a further simplification due to the fact that the holonomy saddles share the common gauge group: the value of (4.26) is by and large independent of the full charged scalar content, other than $\vec{X}_{I}$, so that

$$
\Omega_{K \in \mathcal{N}_{*}}^{\Theta_{*}}=\Omega_{K}^{\Theta_{*}=0},
$$


as we will see this more explicitly in section 4.3 by evaluating these Gaussian integrals. This happens because, in the end, $\Omega_{I}^{\Theta_{*}}$ 's are such that of all charged fields, only one charged scalar multiplet $X_{I}$ meaningfully enters the result.

These two mean that we can formerly and safely replace $\Omega_{I}^{\Theta_{l, m, n}}$ by $\Omega_{I}^{\Theta_{*}=0}$ in (4.33) for all $I$, factor out the $(l, m, n)$ summation for the obvious identity,

$$
\sum_{l, m, n} \delta_{l, m, n}\left(q_{I}\right)=\left|q_{I}\right|^{3}
$$

and arrive at

$$
\Omega_{0}=\sum_{I=1}^{N_{f}}\left|q_{I}\right|^{3} \Omega_{I}^{\Theta_{*}=0},
$$

regardless of details of the $\mathrm{SO}(2)$ theory.

\subsection{Index theorem for $\mathrm{SO}(2)$ Chern-Simons-Matter}

In (4.22) and (4.26), we have successfully reduced the path integrals of $d=3 \mathcal{N}=1 \mathrm{SO}(2)$ Chern-Simons-Matter theories to ordinary Gaussian integrals, while (4.36) has given a simple way to build up the matter contribution $\Omega_{0}$ from holonomy saddles on $\mathbb{T}^{3}$. This means that the essence of the Witten index is transferred again to the matter of understanding the images of the maps, (4.19) and (4.23), much as in (2.32). These are still much cumbersome, so we will discuss how charged-matter integrals can be performed universally, leaving behind only the neutral scalar images $s_{A}$ 's both for $\mathcal{Z}_{\kappa}$ and for $\Omega_{0}$.

For this, we will restrict our attention to $W\left(\rho_{J}, P_{A}\right)$ which is a polynomial of $\rho_{J}=$ $\left|\vec{X}_{J}\right|^{2} / 2$ 's, and find

$$
\mathcal{I}^{3 d}=\kappa \cdot \Omega_{\text {neutral }}+\sum_{I}\left(\frac{q_{I}^{2}}{2} \sum_{a} n_{I}^{(a)} \cdot \operatorname{sgn}\left(W_{I}\left(\Delta_{I}^{(a)}\right)\right)\right) .
$$

Let us explain various quantities in this formula. $\Omega_{\text {neutral }}$ is the $d=1$ Witten index or the twisted partition function for the subsector consisting of the neutral scalar multiplets only, in the sense of section 2. In the double sum on the right hand side, the inner sum is associated with the decomposition of the integral domains into $\cup_{a} \Delta_{I}^{(a)}$, split by $0=W_{I} \equiv \partial_{\rho_{I}} W$, and $n_{I}^{(a)}$ is an effective winding number associated with the neutral part of the Gaussian variable $s_{A}=\partial_{p_{A}} W$ in the domain $\Delta_{I}^{(a)}$. The precise definition of $n_{I}^{(a)}$ is a little more involved than $\Omega_{\text {neutral }}$, which we will discuss presently.

If the neutral sector is absent altogether, in particular, the end result can be obtained by a decoupling limit of theories with the neutral sector, such that the index simplifies to

$$
\mathcal{I}^{3 d}=\kappa+\sum_{I}\left(\frac{q_{I}^{2}}{2} \cdot \operatorname{sgn}\left(W_{I}^{\infty}\right)\right)
$$

where effectively only one "last" domain $\Delta_{I}^{\infty}$ in the $a$-sum contributes with the unit winding number. Note that we have already derived $d=1$ version of the second term, in section 3.3, which differed by the absence of $q_{I}^{2}$ in the summand. We will dedicate this subsection to a proof of these two formulae. 
Evaluation of $\mathcal{Z}_{\kappa}$. Despite the apparently factorized forms of (4.22) and (4.26), the subtlety lies with the integration domain, determined entirely by $W$. Fortunately, the $\vec{y}_{J}$ variables are such that for generic $\mathbf{u}$,

$$
\sigma_{J} \equiv\left(\left(\partial_{\rho_{J}} W\right)^{2}+q_{J}^{2} \mathbf{u}^{2}\right) \rho_{J}=\left|\vec{y}_{J}\right|^{2} / 2
$$

spans $[0,+\infty)$, as $\rho_{J}$ spans $[0,+\infty)$, effectively once if we take the orientation into account. The biggest difference of the gauged version from (2.32) is the absence of winding number in the charged sector. Although $\vec{x}_{J} \rightarrow \vec{y}_{J}$ in (4.19) looks like a map $\mathbb{R}^{2 N_{f}} \times \cdots \rightarrow \tilde{\mathbb{R}}^{2 N_{f}} \times \cdots$, it is in reality a map $\rho_{J} \rightarrow \sigma_{J}$ between $\mathbb{R}_{+}^{N_{f}} \times \cdots$ and $\tilde{\mathbb{R}}_{+}^{N_{f}} \times \cdots$ since $W=W\left(\rho_{I}=\left|\vec{X}_{I}\right|^{2} / 2, \cdots\right)$. Furthermore, the boundary $\cup_{I}\left\{\left|\vec{x}_{I}\right|=0\right\}$ is mapped to the boundary $\cup_{I}\left\{\left|\vec{y}_{I}\right|=0\right\}$.

This means that, regardless of how complicated might be the image of map $\sigma_{J}$ in the wedge $\tilde{\mathbb{R}}_{+}^{N_{f}}$, the Gaussian integral over them eventually always reduces to

$$
\prod_{J} \int_{0}^{\infty} d\left(\vec{y}_{J}^{2} / 2\right) e^{-\left(\vec{y}_{J}\right)^{2} / 2}=\prod_{J} \Gamma(1)=1 .
$$

In particular, this happens regardless of details of $W$, as long as the latter is smooth and at least linearly divergent at large $\rho_{J}$ 's.

What about the integration domain of $s_{A}$ 's? Consider the limit of very large $p_{A}$, while $\vec{y}_{J}$ are held fixed. Decomposing

$$
W=W_{\mathbf{C}}(\rho)+W_{\mathbf{C N}}(\rho ; p)+W_{\mathbf{N}}(p),
$$

and concentrating on the order of magnitude only, we find

$$
s_{A}=\partial_{p_{A}} W=\partial_{p_{A}} W_{\mathbf{N}}+O(1 /|p|)
$$

at large $p$ 's and finite $\vec{y}$ s, for polynomial $W$. The latter suppressed term can be understood as, we have

$$
\left|\partial_{p}\left(W_{\mathbf{C N}}\right)\right| \sim \frac{\left|W_{\mathbf{C N}}\right|}{|p|} \sim \frac{|\vec{y}|}{|p|} \cdot \frac{W_{\mathbf{C N}}}{\sqrt{\left|\partial_{\vec{x}}\left(W_{\mathbf{C}}+W_{\mathbf{C N}}\right)\right|^{2}+\mathbf{u}^{2}|\vec{x}|^{2}}}
$$

at large $p$ and finite $\vec{y}$. So, regardless of the potential complications from $W_{\mathbf{C N}}$, only $W_{\mathbf{N}}$ matters for the contributing $s$ domains. We therefore conclude that the $s$ integrals collapse to a form as in (2.32) for a reduced theory involving $\left\{P_{A}\right\}$ only.

These observations translate to one of the following two possibilities: (1) If the ChernSimons theory contains no neutral field, we must find

$$
\mathcal{Z}_{\kappa}=1
$$

This, in particular, implies that in theories with no neutral scalar multiplet, there is always an $\mathrm{SO}(2)$ symmetric phase in $d=3$, since otherwise the Witten index cannot have $\kappa$ contribution at all. (2) If the Chern-Simons theory comes with neutral matter multiplets, $\mathcal{Z}_{\kappa}$ is computed by the same formula as in $(2.32)$ such that

$$
\mathcal{Z}_{\kappa}=\Omega_{\text {neutral }},
$$


which is obtained by removing the gauge sector and charged matter entirely. If the latter does not give an integral result due to an asymptotic flat direction, one must again scale up $W$ by an arbitrary large and positive constant $C$, which in the end will give an integral result as

$$
\mathcal{Z}_{\kappa}=\mathcal{I}_{\text {neutral }}=\left.\lim _{C \rightarrow \infty} \Omega_{\text {neutral }}\right|_{W \rightarrow C \cdot W} .
$$

For example, a $d=3 \mathcal{N}=2 \mathrm{SO}(2)$ Chern-Simons theory, with the single real $\mathcal{N}=1$ scalar multiplet $\Sigma$ embedded in the $\mathcal{N}=2$ vector and with charged $\mathcal{N}=2$ chirals as the matter sector, one finds $\mathcal{Z}_{\kappa}=-\operatorname{sgn}(\kappa)$, leading to (4.9). Integrating out this auxiliary $\Sigma$, while leaving the $\mathcal{N}=2$ matter sector intact, brings us back to the canonical $\mathcal{Z}_{\kappa}=1$ as in (4.43). In section 4.4, we will confirm (4.45) explicitly for more examples.

In fact, these results can be understood also from $d=3$ Chern-Simons physics. Recall that the $\kappa$-linear contributions come from the pure gauge dynamics at symmetric phases and also that our $\kappa$ is the UV Chern-Simons level. At such symmetric phases, the charged chirals can contribute to the index but only via an additive shift of $\kappa$, which, for our $\mathrm{SO}(2)$ theories, can enter only via $\Omega_{0}$, however, cannot possibly affect $\mathcal{Z}_{\kappa}$. This explains (4.43). On the other hand, the neutral matter multiplets are decoupled from the gauge sector at the symmetric phase, once we integrate out charged matters. This means the ground state sector of such neutral sector will have to be tensored with those from the gauge sector, which in turn explains why one has to find (4.44).

Evaluation of $\Omega_{0}=\sum \Omega_{K}^{\Theta_{*}}$. What can we say for $\Omega_{0}$, or more precisely for $\Omega_{I}^{\Theta_{*}=0}$, where $N_{f} \vec{y}_{J}$ 's are replaced by $\vec{z}_{I}$ and $N_{f}-1 \vec{y}_{K \neq I}$ ? The variable $\vec{z}_{I}$ behaves very similarly as $\vec{y}_{K \neq I}$ 's except that the inverse image of $\vec{z}_{I}=0$ includes the loci $\left\{\vec{x}_{J} \mid W_{I} \equiv \partial_{\rho_{I}} W=0\right\}$, which split $\mathbb{R}_{+}^{N_{f}} \times \mathbb{R}^{\operatorname{dim}(P)}$ into several domains,

$$
\mathbb{R}_{+}^{N_{f}} \times \mathbb{R}^{\operatorname{dim}(P)}=\cup_{a} \Delta_{I}^{(a)},
$$

with a definite sign of $W_{I} \equiv \partial_{I} W$ in each such subdomain. Whether or not such a domain contributes to $\Omega_{I}^{\Theta_{*}=0}$ depends on how its boundary is mapped into the target $\tilde{\mathbb{R}}_{+}^{N_{f}} \times$ $\tilde{\mathbb{R}}^{\operatorname{dim}(S)=\operatorname{dim}(P)}$ obviously spanned by

$$
\lambda_{I}=\vec{z}_{I}^{2} / 2, \quad \sigma_{K \neq I}=\vec{y}_{K}^{2} / 2, \quad s_{A} .
$$

We may safely ignored $\mathbf{v}=\left|q_{I}\right|\left|\vec{x}_{I}\right| \mathbf{u}$ in the following discussion.

For each domain, the boundary $\partial \Delta_{I}^{(a)}$ sitting at finite values of the matter fields consists of $\rho_{I}=0$ or of $\partial_{I} W=0$, so the map in (4.23) from $\left(\rho_{J}, p_{A}\right)$ to $\left(\lambda_{I}, \sigma_{K \neq I}, s_{A}\right)$ induces a map $f_{I}^{(a)}$ such that

$$
f_{I}^{(a)}: \partial \Delta_{I}^{(a)} \hookrightarrow \Upsilon_{I} \equiv\left\{\lambda_{I}=0, \sigma_{K \neq I}, s_{A}\right\}
$$

since $\lambda_{I}=\left(\partial_{I} W\right)^{2} \rho_{I}$. Note that each of $\Upsilon_{I}$ can be regarded as a copy of $\tilde{\mathbb{R}}_{+}^{N_{f}-1} \times \tilde{\mathbb{R}}^{\operatorname{dim}(S)}$. Here we are freezing $\mathbf{v}=\left|q_{I}\right|\left|\vec{x}_{I}\right| \mathbf{u}$ to a generic constant value, as its precise value does not affect the classification below and the result of the integral below. 
There are two logical possibilities where domain $\Delta_{I}^{(a)}$ can contribute to $\Omega_{0}$ : (i) $f_{I}^{(a)}$ covers the entire $\Upsilon_{I} n_{I}^{(a)}$ times, or (ii) $f_{I}^{(a)}$ does not cover the entire $\Upsilon_{I}$ but does so $n_{I}^{(a)}$ times if we scale up $W \rightarrow C \cdot W$ with an arbitrarily large positive number $C$. When either of this happens, assuming polynomial form of $W$, one can see that the image of $\Delta_{I}^{(a)}$ under the map (4.23) is the entire $\tilde{\mathbb{R}}_{+}^{N_{f}} \times \tilde{\mathbb{R}}^{\operatorname{dim}(S)}$, so each Gaussian integral over $\Delta_{I}^{(a)}$ contributes to $\Omega_{0}$ an additive piece

$$
\frac{1}{2\left|q_{I}\right|} n_{I}^{(a)} \cdot \operatorname{sgn}\left(W_{I}^{(a)}\right)
$$

although this requires the scaling in case of (ii). Summation over the domains give

$$
\Omega_{I}^{\Theta_{*}=0}=\frac{1}{2\left|q_{I}\right|} \sum_{a} n_{I}^{(a)} \cdot \operatorname{sgn}\left(W_{I}^{(a)}\right),
$$

while the entire $\Omega_{0}$ is constructed via (4.36) as

$$
\Omega_{0}=\sum_{I}\left(\frac{q_{I}^{2}}{2} \sum_{a} n_{I}^{(a)} \cdot \operatorname{sgn}\left(W_{I}^{(a)}\right)\right) .
$$

This concludes the proof of (4.37).

Note that each and every term in the sum is a multiple of $1 / 2$; this is consistent with the fact that the Chern-Simons level shifts in unit of $1 / 2$ due to the one-loop correction by matters. Such a would-be one-loop contribution is embedded in $\Omega_{0}$ in this path-integral computation, as was commented earlier.

What are the other possibilities other than (i) and (ii) above? The typical reason why $\Delta_{I}^{(a)}$ fails to contribute is that the map $f_{I}^{(a)}$ folds its image in $\Upsilon_{I}$ in such a way $n_{I}^{(a)}=0$ effectively. Note that $\rho_{K \neq I}=0$ always maps to $\sigma_{K}=0$. This implies that there is no winding number to consider in the charged matter sector. Instead, the winding number comes from how $p_{A}$ gets mapped to $s_{A}$, along $\partial \Delta_{I}^{(a)}$. Therefore, even though things are a little more complicated compared to the $\mathcal{Z}_{\kappa}$ computation, $\Omega_{0}$ is again dictated by the winding numbers which are by and large determined by the neutral matter sector. In fact, if the neutral sector is absent, at most one $\Delta_{I}^{(a)}$ for each $I$ may contribute because of this, as we will see below.

When there is only one $P$, on the other hand, the only possible winding number is either $n_{I}^{(a)}= \pm 1$ or $n_{I}^{(a)}=0$. To determine $n_{I}^{(a)}$, all one has to do is to trace how $s$ grows as one follows the two disjoint asymptotic ends of $\partial \Delta_{I}^{(a)}$. If $s$ grows to the two opposite infinities (after $C$ scaling if necessary), we have $n_{I}^{(a)}= \pm 1$ and if not $n_{I}^{(a)}=0$. In section 4.4, we will deal with a class of examples of this kind, and confirm what we described here somewhat abstractly by a more explicit computation.

If the neutral field $P$ 's are absent altogether, the images $\left(\lambda_{I}, \sigma_{K \neq I}\right)$ of these domains simplify further. With $\mathbf{u}^{2}$ present, the inverse image of $\sigma_{K \neq I}=0$ is simply $\rho_{K \neq I}=0$. This means that most of the above domains $\Delta_{I}^{(a)}$ are such that two adjacent locus of $\partial_{I} W=0$ are both mapped to $\lambda_{I}=0$ and that the image folds itself in $\tilde{\mathbb{R}}_{+}^{N_{f}}$. Once we take into account the orientation, the integration in (4.26) over such $\Delta_{I}^{(a)}$ would vanish; 
and the only outermost domain, with one boundary mapped to $\lambda_{I}=\infty$, contributes to the integral $\Omega_{I}^{\Theta_{*}=0}$.

Calling this last domain $\Delta_{I}^{\infty}$, we have the universal contribution,

$$
\frac{1}{2\left|q_{I}\right| \cdot \operatorname{sgn}\left(W_{I}^{\infty}\right)}
$$

where the sign is unambiguously determined on the same outermost domain in space $\mathbb{R}_{+}^{N_{f}}$, spanned by $\rho_{J}$. This in turn leads to

$$
\Omega_{0}=\sum_{I} \frac{q_{I}^{2}}{2} \cdot \operatorname{sgn}\left(W_{I}^{\infty}\right),
$$

as claimed in (4.38).

\subsection{Examples, wall-crossings, and dualities}

Here we will give a cursory look at a few examples. The goal is to solidify the general formula by performing the explicit integration of the Gaussian formulae (4.22) and (4.26), and in particular to give a little more concrete picture of the index formula (4.37) by studying explicit examples. We will close with $3 \mathrm{~d} \mathcal{N}=1$ duality pairs by showing how the index counting is consistent with the claimed dualities.

A word of caution is in order. Recall that the Witten index has an inherent sign ambiguity. Sometimes, we may use $(-1)^{F} \rightarrow(-1)^{J}$ with $J$ an unambiguously defined integral fermion number, but no such is naturally available in our class of theories with Majorana supercharges. Instead, we have adopted a sign convention consistent with the standard Morse theory counting or the natural winding number counting. This leads to $\mathcal{Z}_{\kappa}=1$ for a pure $\mathcal{N}=1 \mathrm{SO}(2)$ gauge theory, in particular. It is worthwhile to note that extending the same sign convention to $d=3 \mathcal{N}=2$ Chern-Simons theories, with a single real $\mathcal{N}=1$ scalar multiplet $\Sigma$ as part of the $\mathcal{N}=2$ vector multiplet, we have $\mathcal{Z}_{\kappa}=-\operatorname{sgn}(\kappa)$. For example, the pure $\mathcal{N}=2 \mathrm{SO}(2)$ Chern-Simons theory would have the index $-|\kappa|$ in our convention.

SO(2) without neutral matters. For $\mathrm{SO}(2)$ theories with charged matters only and a generic superpotential of the form $W\left(\rho_{I}\right)$, the Gaussian integrals to be performed for $\Omega_{I}^{\Theta_{*}=0}$ are in fact identical to the ones already computed for $d=1$ in section 3.3. For $N_{f}$ matters, $\Omega_{I}^{\Theta_{*}=0}$ was originally computed in the $d=1$ context, which we quote here again

$$
\Omega_{I}^{\Theta_{*}=0}=\frac{1}{2\left|q_{I}\right|} \cdot \operatorname{sgn}\left(W_{I}^{\infty}\right)
$$

and which went into the theorem (4.38). The simplest theory is the quadratic $W=$ $\sum_{J=1}^{N_{f}} m_{J} \rho_{J}$, which leads to

$$
\Omega_{I}^{\Theta_{*}=0}=\frac{1}{2\left|q_{I}\right|} \cdot \operatorname{sgn}\left(m_{I}\right) .
$$


Another example is the quartic $W=c_{W}(\rho-\xi)^{2} / 2$ with a single charge $q$ complex scalar multiplet. We have

$$
\Omega_{I}^{\Theta_{*}=0}=\frac{1}{2|q|} \cdot \operatorname{sgn}\left(c_{W}\right)
$$

for this example.

What do change are the holonomy saddles, which are more numerous in $d=3$ than in $d=1$; the net effect of them is now

$$
\Omega^{1 d}=\sum_{I}\left|q_{I}\right| \Omega_{I}^{\Theta_{*}=0} \quad \rightarrow \quad \Omega_{0}=\sum_{I}\left|q_{I}\right|^{3} \Omega_{I}^{\Theta_{*}=0}
$$

with the common quantities $\Omega_{I}^{\Theta_{*}=0}$ shared by $d=1$ and $d=3$. This counting (4.36) has been incorporated into the formulae (4.37) and (4.38). The truly new part for $d=3$ theories is $\mathcal{Z}_{\kappa}$, which in the previous subsection we have shown to be 1 universally if the neutral matters entirely are absent.

Combining these, one finds, for example,

$$
\mathcal{I}^{3 d}=\kappa+\frac{1}{2}|q|^{2} \operatorname{sgn}\left(c_{W}\right)
$$

for the above quartic theory with a single charge $q$ complex scalar multiplet, regardless of the sign of $\xi$. Therefore, there is a wall-crossing at $c_{W}=0$ but none at $\xi=0$. We can view this result in the $d=3$ perspective as follows. For $\xi>0$ where we have both the symmetric and the broken vacua, this result can be more physically written as

$$
\mathcal{I}_{(\xi>0)}^{3 d}=\left(\kappa-\frac{1}{2}|q|^{2} \operatorname{sgn}\left(c_{W} \xi\right)\right)+|q|^{2} \operatorname{sgn}\left(c_{W} \xi\right),
$$

where the last term comes from the Higgs vacuum at $\rho=\xi ; q^{2}$ is due to so-many holonomy choices along the spatial $\mathbb{T}^{2}$. The first two terms are interpreted due to the quantum vacua sitting at the symmetric phase $\rho=0$. The local matter multiplet has the mass $-c_{W} \xi$, hence the Chern-Simons level is shifted in the infrared by $-q^{2} \operatorname{sgn}\left(c_{W} \xi\right) / 2$. With $\xi<0$, where only symmetric vacua survive, the shift of the Chern-Simons level is now $-\frac{1}{2}|q|^{2} \operatorname{sgn}\left(c_{W} \xi\right)=$ $\frac{1}{2}|q|^{2} \operatorname{sgn}\left(c_{W}\right)$. The entire index arises from the infrared Chern-Simons level,

$$
\mathcal{I}_{(\xi<0)}^{3 d}=\kappa+\frac{1}{2}|q|^{2} \operatorname{sgn}\left(c_{W}\right),
$$

as expected.

We can in fact expand this discussion to theories with an arbitrary number of charged matters, equipped with a radial superpotential $W\left(\rho_{I}\right)$. The index we compute by a direct path integral would be

$$
\mathcal{I}^{3 d}=\kappa+\sum_{I} \frac{q_{I}^{2}}{2} \cdot \operatorname{sgn}\left(W_{I}^{\infty}\right),
$$

where the sign of $W_{I}=\partial_{\rho_{I}} W$ is measured at the corner of $\rho_{I} \rightarrow \infty, \rho_{J(\neq I)} \rightarrow 0^{+}$. Again we can write this as

$$
\mathcal{I}^{3 d}=\left(\kappa+\sum_{I} \frac{q_{I}^{2}}{2} \cdot \operatorname{sgn}\left(m_{I}\right)\right)+\sum_{I} \frac{q_{I}^{2}}{2} \cdot\left(\operatorname{sgn}\left(W_{I}^{\infty}\right)-\operatorname{sgn}\left(m_{I}\right)\right),
$$


where the first captures the shift of level $\kappa$ in the symmetric phase, with the effective mass $m_{I}$ of field $X_{I}$ at the symmetric vacuum. Among other things, this implies that an $\mathrm{SO}(2)$ symmetric vacuum always exists, regardless of the details of $W\left(\rho_{I}\right)$. For generic $W$, the second piece, which is always integral, may be attributed to additional Higgs vacua.

These $m_{I}$ 's, which parameterize $W$, affect the infrared Chern-Simons level, which in turn affects the contribution to the index from the symmetric phase. On the other hand, the same parameters can affect where and how the broken vacua appear, and therefore also affect the contribution from Higgs vacua. This latter effect is reflected in the second part of the above index formula. In the end, however, these parameter-dependences of individual contributions cancel out, much as in the above quartic model, and the index is independent of these $m_{I}$ 's. Instead, the wall-crossing happens only when the asymptotics of the above superpotential changes, in a very limited sense via $\operatorname{sgn}\left(W_{I}\right)$ 's. This should be contrasted to $(2.32)$ where one can in principle have an arbitrarily high winding number as the index. This is the consequence of the fact that the effective domain of matter fields is $\mathbb{R}_{+}^{N_{f}}$ rather than $\mathbb{R}^{2 N_{f}}$, as far as the map $d W$ induced by the superpotential goes.

SO(2) with neutral matters. With neutral matters present, we have seen in the previous subsection that the index formula changes substantially in such a way certain winding numbers enter the final formula. Here we will take one last example of type with a single neutral matter $P$ and the superpotential

$$
W=P \cdot K(\rho)+L(\rho)+F(P) .
$$

This may be regarded as a generalization of the $\mathbb{W} \mathbb{C P}^{N_{f}-1}$ model in section 3.3 , which corresponds to the choice

$$
K(\rho)=\sum_{I=1}^{N_{f}} q_{I} \rho_{I}, \quad L(\rho)=0, \quad F(P)=-\xi P .
$$

In $d=3$, even if we start with the $\mathbb{W} \mathbb{C P}$ model, the one-loop corrections to the superpotential will typically generate quadratic terms in $F(P)$, as we will see at the end of this subsection. Thus, here we consider a generic polynomial $K(\rho), L(\rho)$ and $F(P)$, which can include such one-loop corrections. With this general class of examples, we aim at understanding quantities that appear in the index theorem (4.37) a little more concretely.

As before, the Witten index consists of two parts: $\kappa \cdot \mathcal{Z}_{\kappa}$ and $\Omega_{0}=\sum_{\Theta_{*}} \sum_{I} \Omega_{I}^{\Theta_{*}}$. We demonstrate the explicit computation of those two contributions and confirm the index theorem in the previous subsection. First let us look at $\kappa \cdot \mathcal{Z}_{\kappa}$, given by

$$
\kappa \cdot \mathcal{Z}_{\kappa}=\frac{\kappa}{(2 \pi)^{N_{f}+\frac{7}{2}}} \int d^{3} \mathbf{v} d s d^{2 N_{f}} y \exp \left(-\frac{1}{2} s^{2}-\frac{1}{2} \sum_{J} \vec{y}_{J}^{2}\right),
$$

where

$$
\mathbf{v}=\mathbf{u}, \quad s=K(\rho)+F^{\prime}(p), \quad \vec{y}_{J}=\sqrt{q_{J}^{2} \mathbf{u}^{2}+\left(p K_{J}(\rho)+L_{J}(\rho)\right)^{2}} \cdot \vec{x}_{J},
$$


with $K_{J}=\frac{\partial K}{\partial \rho_{J}}, L_{J}=\frac{\partial L}{\partial \rho_{J}}$ and $F^{\prime}(p)=\frac{d F}{d p}$. We note that the slice of constant $\sigma_{J}=\vec{y}_{J}^{2} / 2$ and constant $\mathbf{v}^{2}$ is determined by

$$
\sigma_{J}=\left(q_{J}^{2} \mathbf{v}^{2}+\left(p K_{J}(\rho)+L_{J}(\rho)\right)^{2}\right) \rho_{J}, \quad \text { for } J=1, \ldots, N_{f},
$$

in the $(\rho, p)$-space.

For the moment, we assume that, for given $\sigma_{J}$ and $\mathbf{v}^{2},(4.66)$ has the unique solution $\rho_{J}=f_{J}\left(p ; \sigma, \mathbf{v}^{2}\right)$ for $J=1, \cdots, N_{f}$. Then $s$ is solely a function of $p$ :

$$
s=K\left(f\left(p ; \sigma, \mathbf{v}^{2}\right)\right)+F^{\prime}(p),
$$

which determines the image of the integration domain of $p \in(-\infty, \infty)$ on the $s$-space as follows:

$$
s \in\left(\lim _{p \rightarrow-\infty}\left[K\left(f\left(p ; \sigma, \mathbf{v}^{2}\right)\right)+F^{\prime}(p)\right], \lim _{p \rightarrow+\infty}\left[K\left(f\left(p ; \sigma, \mathbf{v}^{2}\right)\right)+F^{\prime}(p)\right]\right) .
$$

Since $\rho_{J}=f_{J}\left(p ; \sigma, \mathbf{v}^{2}\right)$ remains finite when $p$ goes to $\pm \infty, K(\rho)$, a polynomial of $\rho_{J}$, is also finite and negligible compared to $F^{\prime}(p)$. Thus, we have the following integration domain of $s$ for fixed $\sigma_{J}$ and $\mathbf{v}^{2}$ :

$$
s \in\left(\lim _{p \rightarrow-\infty} F^{\prime}(p), \lim _{p \rightarrow+\infty} F^{\prime}(p)\right)
$$

which is nothing but a consequence of (4.41). In general, (4.66) does not give rise to the unique solution $\rho_{J}=f_{J}\left(p ; \sigma, \mathbf{v}^{2}\right)$ because the codimension- $N_{f}$, i.e., one-dimensional, subspace defined by (4.66) in the $(\rho, p)$-space is rather arbitrary. Nevertheless, (4.65) defines a map from this one-dimensional space to the Gaussian variable $s$, whose image is given by, with $\sigma_{J}, \mathbf{v}^{2}$ fixed,

$$
s \in\left\{K(\rho)+F^{\prime}(p) \mid \sigma_{J}=\left(q_{J}^{2} \mathbf{v}^{2}+\left(p K_{J}(\rho)+L_{J}(\rho)\right)^{2}\right) \rho_{J}\right\} .
$$

Since (4.66) restricts $\rho_{J}$, and $K(\rho)$ accordingly, to be finite, the image on $s$ again spans (4.69).

Note that (4.69) is independent of the fixed values of $\sigma_{J}$ and $\mathbf{v}^{2}$. Thus, the entire path integral decomposes into the decoupled integrals over $\sigma_{J}, \mathbf{v}$ and $s$. The integration domain of each $\sigma_{J}$ is nothing but $\mathbb{R}_{+}$while that of $\mathbf{v}$ is $\mathbb{T}^{3}$ of volume $(2 \pi)^{3}$. Therefore, we are left with the index of the single neutral matter,

$$
\begin{aligned}
& \kappa \cdot \mathcal{Z}_{\kappa}=\frac{\kappa}{\sqrt{2 \pi}} \int_{F^{\prime}(p \rightarrow-\infty)}^{F^{\prime}(p \rightarrow \infty)} d s e^{-\frac{1}{2} s^{2}} \\
& =\left\{\begin{array}{cccc}
+\kappa, & \lim _{p \rightarrow-\infty} F^{\prime}(p) \rightarrow-\infty & \text { and } & \lim _{p \rightarrow \infty} F^{\prime}(p) \rightarrow+\infty, \\
0, & \lim _{p \rightarrow-\infty} F^{\prime}(p) \rightarrow \pm \infty & \text { and } & \lim _{p \rightarrow \infty} F^{\prime}(p) \rightarrow \pm \infty, \\
-\kappa, & \lim _{p \rightarrow-\infty} F^{\prime}(p) \rightarrow+\infty & \text { and } & \lim _{p \rightarrow \infty} F^{\prime}(p) \rightarrow-\infty
\end{array}\right.
\end{aligned}
$$

which agrees with the index theorem (4.45). 
Next, we move on to $\Omega_{0}=\sum_{\Theta_{*}} \sum_{I} \Omega_{I}^{\Theta_{*}}$, which can be inferred entirely from

$$
\Omega_{I}^{\Theta_{*}=0}=\frac{1}{(2 \pi)^{N_{f}+\frac{5}{2}}} \int d^{3} \mathbf{v} d s d^{2} z_{I} d^{2\left(N_{f}-1\right)} y \frac{1}{\left|q_{I}\right| \operatorname{sgn}\left(W_{I}\right)\left|\vec{z}_{I}\right|} \exp \left(-\tilde{V}_{\text {bosonic }}\right)
$$

with $\tilde{V}_{\text {bosonic }}=\left(\mathbf{v}^{2}+s^{2}+\vec{z}_{I}^{2}+\sum_{J \neq I} \vec{y}_{J}^{2}\right) / 2$ as before, where

$$
\begin{aligned}
\mathbf{v} & =\left|q_{I}\right|\left|\vec{x}_{I}\right| \mathbf{u}=\left|q_{I}\right| \sqrt{2 \rho_{I}} \mathbf{u}, & s & =K(\rho)+F^{\prime}(p), \\
\vec{z}_{I} & =\left(p K_{I}(\rho)+L_{I}(\rho)\right) \vec{x}_{I}, & \vec{y}_{J(\neq I)} & =\sqrt{q_{J}^{2} \mathbf{u}^{2}+\left(p K_{J}(\rho)+L_{J}(\rho)\right)^{2}} \cdot \vec{x}_{J} .
\end{aligned}
$$

Again the slice of constant $\lambda_{I}=\vec{z}_{I}^{2} / 2, \sigma_{J(\neq I)}=\vec{y}_{J}^{2} / 2$ and $\mathbf{v}^{2}$ in the $(\rho, p)$-space is determined by

$$
\begin{aligned}
\lambda_{I} & =\left(p K_{I}(\rho)+L_{I}(\rho)\right)^{2} \rho_{I}, \\
\sigma_{J(\neq I)} & =\left(\frac{q_{J}^{2}}{q_{I}^{2}} \frac{\mathbf{v}^{2}}{2 \rho_{I}}+\left(p K_{J}(\rho)+L_{J}(\rho)\right)^{2}\right) \rho_{J} .
\end{aligned}
$$

In particular, (4.74) is equivalent to the following two equations

$$
p K_{I}(\rho)+L_{I}(\rho)= \pm \sqrt{\frac{\lambda_{I}}{\rho_{I}}},
$$

each of which, together with (4.75), defines the one-dimensional subspace in the $(\rho, p)$ space. Let us denote those one-dimensional spaces by $\mathcal{S}_{\lambda_{I}, \sigma_{J}}^{( \pm)}$. For $(\rho, p) \in \mathcal{S}_{\lambda_{I}, \sigma_{J}}^{( \pm)}, \pm W_{I}=$ $\pm\left(p K_{I}+L_{I}\right)>0$ respectively. (4.73) maps $(\rho, p) \in \mathcal{S}_{\lambda_{I}, \sigma_{J}}^{( \pm)}$to the Gaussian variable $s$ as follows:

$$
s \in\left\{K(\rho)+F^{\prime}(p) \mid(\rho, p) \in \mathcal{S}_{\lambda_{I}, \sigma_{J}}^{( \pm)}\right\}, \quad \lambda_{I}, \sigma_{J(\neq I)}, \mathbf{v}^{2} \text { fixed }
$$

In the previous subsection, we argued that the domain of $(\rho, p): \mathbb{R}_{+}^{N_{f}} \times \mathbb{R}$ is split into several subdomains $\cup_{a} \Delta_{I}^{(a)}$, each of which has a definite sign of $W_{I}$. Indeed, each $\mathcal{S}_{\lambda_{I}, \sigma_{J}}^{( \pm)}$ with extra directions from $\lambda_{I}$ and $\sigma_{J(\neq I)}$ spans each $\Delta_{I}^{( \pm)}$; i.e.,

$$
\Delta_{I}^{( \pm)}=\left\{(\rho, p) \mid(\rho, p) \in \mathcal{S}_{\lambda_{I}, \sigma_{J}}^{( \pm)} \text {for } \lambda_{I} \in \mathbb{R}_{+}, \sigma_{J(\neq I)} \in \mathbb{R}_{+}\right\}
$$

whose boundary is given by

$$
\partial \Delta_{I}^{( \pm)}=\lim _{\lambda_{I} \rightarrow 0} \Delta_{I}^{( \pm)}=\left\{(\rho, p) \mid(\rho, p) \in \mathcal{S}_{0, \sigma_{J}}^{ \pm} \text {for } \sigma_{J(\neq I)} \in \mathbb{R}_{+}\right\} .
$$

To determine the image (4.77), we need to examine where the asymptotic regions of $\mathcal{S}_{\lambda_{I}, \sigma_{J}}^{( \pm)}$ are mapped to.

We first note that, for fixed $\sigma_{J(\neq I)}$ and $\mathbf{v}^{2}, \rho_{J(\neq I)}$ may diverge if

$$
\rho_{I} \rightarrow \infty, \quad W_{J}=p K_{J}(\rho)+L_{J}(\rho) \rightarrow 0
$$


simultaneously, which does not happen unless $K(\rho)$ and $L(\rho)$ are fine-tuned. Thus, from now on, we assume that the potential is generic such that $\rho_{J(\neq I)}$ is always finite in $\mathcal{S}_{\lambda_{I}, \sigma_{J}}^{( \pm)}$. Then the asymptotic regions of $\mathcal{S}_{\lambda_{I}, \sigma_{J}}^{( \pm)}$are characterized by either $\rho_{I}$ or $p$ being infinity. Indeed, from (4.74), one can see that there are two asymptotic regions of each $\mathcal{S}_{\lambda_{I}, \sigma_{J}}^{( \pm)}$:

$$
\begin{cases}\rho_{I} \rightarrow 0, & p \rightarrow s_{I}^{( \pm)} \infty \\ \rho_{I} \rightarrow \infty, & p \rightarrow p^{*}\end{cases}
$$

where $s_{I}^{( \pm)}$is defined by

$$
s_{I}^{( \pm)}= \pm \operatorname{sgn} \lim _{\rho_{I} \rightarrow 0}\left(\left.K_{I}(\rho)\right|_{S_{\lambda_{I}, \sigma_{J}}^{ \pm}}\right)
$$

$p^{*}$ is either finite or infinite depending on the details of $K(\rho)$ and $L(\rho)$. In particular, assuming

$$
K(\rho) \approx \alpha \rho_{I}^{k}, \quad L(\rho) \approx \beta \rho_{I}^{l},
$$

for large $\rho_{I}$, i.e., the highest order terms in $\rho_{I}$ is independent of the other $\rho_{J(\neq I)}, p^{*}$ is given by

$$
p^{*}=\lim _{\rho_{I} \rightarrow \infty}\left(\left.p\right|_{\mathcal{S}_{\lambda_{I}, \sigma_{J}}^{( \pm)}}\right) \approx-\frac{l \beta}{k \alpha} \rho_{I}^{l-k}
$$

which diverges if $l>k$ and remains finite if $k \geq l$. See figure 2(a) and (b).

The image on the $s$-space is thus given by

$$
\begin{aligned}
s & \in\left\{K(\rho)+F^{\prime}(p) \mid(\rho, p) \in \mathcal{S}_{\lambda_{I}, \sigma_{J}}^{( \pm)}\right\} \\
& =\left(\lim _{p \rightarrow-\infty} F^{\prime}(p), \lim _{\rho_{I} \rightarrow \infty} K(\rho)+\lim _{p \rightarrow p^{*}} F^{\prime}(p)\right) \cup\left(\lim _{\rho_{I} \rightarrow \infty} K(\rho)+\lim _{p \rightarrow p^{*}} F^{\prime}(p), \lim _{p \rightarrow+\infty} F^{\prime}(p)\right) .
\end{aligned}
$$

with $\operatorname{sgn}\left(W_{I}\right)=s_{I}^{(-)}$or $\operatorname{sgn}\left(W_{I}\right)=s_{I}^{(+)}$for each segment respectively. Again this is independent of the fixed values of $\lambda_{I}, \sigma_{J(\neq I)}$ and $\mathbf{v}^{2}$. Thus, the entire path integral decomposes into the decoupled Gaussian integrals over $\vec{z}_{I}, \vec{y}_{J(\neq I)}$, v and $s$. In the end, we are left with

$$
\begin{aligned}
\Omega_{I}^{\Theta_{*}=0}= & \frac{s_{I}^{(-)}}{2\left|q_{I}\right|} \frac{1}{\sqrt{2 \pi}} \int_{F^{\prime}(p \rightarrow-\infty)}^{K\left(\rho_{I} \rightarrow \infty\right)+F^{\prime}\left(p \rightarrow p^{*}\right)} d s e^{-\frac{1}{2} s^{2}} \\
& +\frac{s_{I}^{(+)}}{2\left|q_{I}\right|} \frac{1}{\sqrt{2 \pi}} \int_{K\left(\rho_{I} \rightarrow \infty\right)+F^{\prime}\left(p \rightarrow p^{*}\right)}^{F^{\prime}(p \rightarrow+\infty)} d s e^{-\frac{1}{2} s^{2}}
\end{aligned}
$$

where each integral with prefactor $\frac{1}{\sqrt{2 \pi}}$ gives the winding number of the map

$$
f_{I}^{( \pm)}: \partial \Delta_{I}^{( \pm)} \quad \hookrightarrow \quad\left\{\lambda_{I}=0, \sigma_{J(\neq I)}, s\right\}
$$


(a) $k=\operatorname{deg} K \geq \operatorname{deg} L=l$

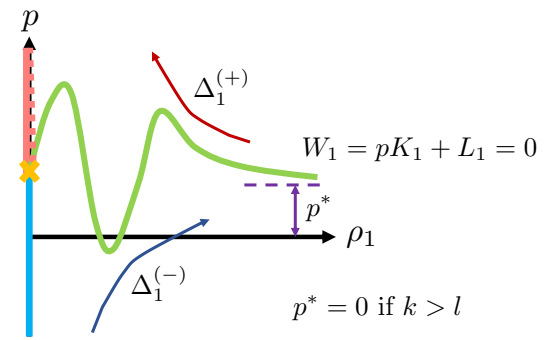

(c) when $F(p)$ has higher-order terms

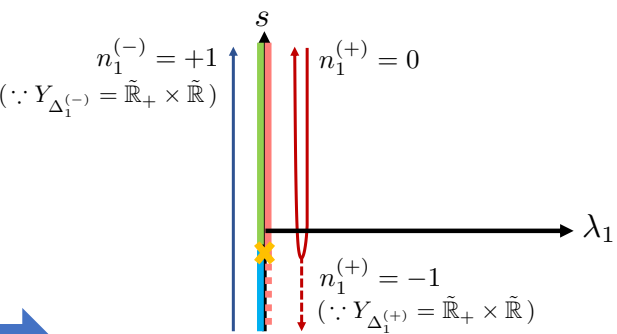

(b) $k<l$

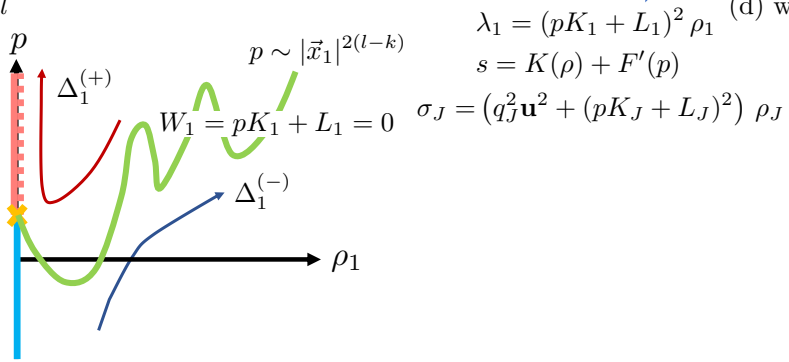

(d) when $F(p)$ is linear

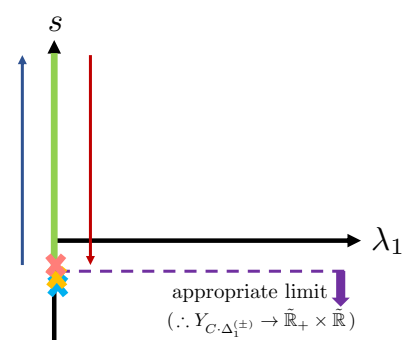

Figure 2. The domain decomposition and mapping under reparameterization (4.23) for $\Omega_{I=1}$ of $W=P \cdot K(\rho)+L(\rho)+F(P)$ theory. The angular part and other directions such as $\rho_{I \geq 2}, \sigma_{I \geq 2}$ are ignored to avoid the clutter.

induced from (4.73), which is either \pm 1 or 0 in this case. See figure 2(c). Taking the holonomy saddles into account, we have the following result for entire $\Omega_{0}$ :

$$
\Omega_{0}=\sum_{I=1}^{N_{f}} \frac{q_{I}^{2}}{2} \sum_{a= \pm} s_{I}^{(a)} n_{I}^{(a)}
$$

where

$$
\begin{aligned}
& n_{I}^{(+)}=\frac{1}{\sqrt{2 \pi}} \int_{K\left(\rho_{I} \rightarrow \infty\right)+F^{\prime}\left(p \rightarrow p^{*}\right)}^{F^{\prime}(p \rightarrow+\infty)} d s e^{-\frac{1}{2} s^{2}}, \\
& n_{I}^{(-)}=\frac{1}{\sqrt{2 \pi}} \int_{F^{\prime}(p \rightarrow-\infty)}^{K\left(\rho_{I} \rightarrow \infty\right)+F^{\prime}\left(p \rightarrow p^{*}\right)} d s e^{-\frac{1}{2} s^{2}},
\end{aligned}
$$

which confirms the index theorem (4.37).

So far we have considered a linear coupling of $P$ to a polynomial of $\rho$, which however can be generalized to higher order interactions. In the above linear interaction example, the domain of the original integration splits into two region divided by a codimension-one space defined by $W_{I}=0$. On the other hand, with the generalized superpotential

$$
W=\sum_{m \leq M} P^{m} \cdot K^{(m)}(\rho)+F(p)
$$

now the original integration domain splits in a much more complicated way. For instance, $W_{I}=0$ has at most $M$ solutions of $P$ for fixed $\rho_{I}$. Those solutions can merge, split 
and cross each other along the way that $\rho_{I}$ changes, and the trajectories of such solutions define the codimension-one spaces that divide the domain into several subdomains. Note that, among those subdomains, only those unbounded along the $\rho_{I} \rightarrow \infty$ direction can give nontrivial contributions to the integral; i.e., any bounded subdomain gives a vanishing contribution because its image under the map (4.23) is folded and effectively vanishes. Thus, we only need to consider unbounded subdomains and the boundaries between them stretched to $\rho_{I} \rightarrow \infty$. There are at most $M$ such infinitely stretched boundaries. Their asymptotic values of $p$ for $\rho_{I} \rightarrow \infty$ can be denoted by $p^{(n)}$ with $n=1, \ldots, N \leq M$ where $N+1$ is the number of unbounded subdomains. Each subdomain contributes to the integral by its winding number \pm 1 if

$$
\begin{gathered}
\lim _{\substack{\rho_{I} \rightarrow 0, p \rightarrow-\infty}} \operatorname{sgn}(s) \neq \lim _{\substack{\rho_{I} \rightarrow \infty \\
p \rightarrow p^{(1)}}} \operatorname{sgn}(s), \\
\lim _{\substack{\rho_{I} \rightarrow \infty, p \rightarrow p^{(N)}}} \operatorname{sgn}(s) \neq \lim _{\substack{\rho_{I} \rightarrow 0, p \rightarrow \infty}} \operatorname{sgn}(s)
\end{gathered}
$$

for the first and the last subdomain respectively and

$$
\lim _{\substack{\rho_{I} \rightarrow \infty, p \rightarrow p^{(n-1)}}} \operatorname{sgn}(s) \neq \lim _{\substack{\rho_{I} \rightarrow \infty, p \rightarrow p^{(n)}}} \operatorname{sgn}(s)
$$

for the others.

Lastly, we conclude the discussion by demonstrating an explicit example with definite winding numbers. For example, let us choose $K(\rho), L(\rho)$ and $F(p)$ as follows:

$$
K(\rho)=\sum_{I=1}^{N_{f}} q_{I} \rho_{I}, \quad L(\rho)=\sum_{I=1}^{N_{f}} m_{I} \rho_{I}, \quad F(P)=-\xi P+\frac{1}{2} m P^{2}+\frac{1}{2} \alpha P|P| .
$$

Although $F(p)$ here, strictly speaking, is not a polynomial, the above discussion is still valid for this example. Using the results obtained above, we find that $\kappa \cdot \mathcal{Z}_{k}$ and $\Omega_{I}^{\Theta_{*}=0}$ for this model are as follows:

$$
\kappa \cdot \mathcal{Z}_{\kappa}= \begin{cases}+\kappa, & |\alpha|<m \\ 0, & -|\alpha|<m<|\alpha| \\ -\kappa, & m<-|\alpha|\end{cases}
$$

and

$$
\Omega_{I}^{\Theta_{*}=0}= \begin{cases}-\frac{1}{2} \frac{1}{\left|q_{I}\right|}, & |\alpha|<m, \\ 0, & -\operatorname{sign}\left(q_{I}\right) \alpha<m<\operatorname{sign}\left(q_{I}\right) \alpha, \\ -\frac{1}{\left|q_{I}\right|}, & \operatorname{sign}\left(q_{I}\right) \alpha<m<-\operatorname{sign}\left(q_{I}\right) \alpha, \\ -\frac{1}{2} \frac{1}{\left|q_{I}\right|}, & m<-|\alpha| .\end{cases}
$$

Eqs. (4.95) and (4.96) with the holonomy saddles lead to the following result for the 3d index:

$$
\mathcal{I}^{3 d}= \begin{cases}\kappa-\frac{1}{2} \sum_{I} q_{I}^{2}, & |\alpha|<m, \\ -\sum_{I \in \hat{I}_{ \pm}} q_{I}^{2}, & \pm \alpha<m<\mp \alpha, \\ -\kappa-\frac{1}{2} \sum_{I} q_{I}^{2}, & m<-|\alpha|\end{cases}
$$


where $\hat{I}_{ \pm}$are sets of the indices having positive/negative charges respectively; i.e.,

$$
\hat{I}_{ \pm}=\left\{I \mid \pm q_{I}>0\right\} .
$$

We note that there are wall-crossings at $m= \pm \alpha$. On the other hand, the index is independent of $\xi$.

In particular, if we set

$$
\alpha=-\sum_{I=1}^{N_{f}} \frac{q_{I}\left|q_{I}\right|}{2},
$$

this example captures the index of the $\mathbb{W} \mathbb{C P}^{N_{f}-1}$ model with massive $P$ and the one-loop corrected superpotential. For the $\mathbb{W} \mathbb{C P}^{N_{f}-1}$ model, in fact, the one-loop correction to the superpotential is given by

$$
-\frac{1}{2} \sum_{I=1}^{N_{f}}\left(q_{I} P+m_{I}\right)\left|q_{I} P+m_{I}\right|,
$$

which leads to a more complicated vacuum equation. However, we have seen that only the asymptotics of $F(p)$ is important as long as we compute the Witten index. Hence, the index of $\mathbb{W} \mathbb{C P}^{N_{f}-1}$ can be captured by more simpler one like (4.94).

One can easily check (4.97) for the $\mathbb{W} \mathbb{C} \mathbb{P}^{N_{f}-1}$ model with massive $P$ in some limiting cases. First we assume $m_{I}$ are non-zero but very small compared to other parameters. For $|m| \gg|\alpha|$, a vacuum sits either at $P=\frac{\xi}{m}$ or at $P=0$. At $P=\frac{\xi}{m}$, massive $P$ is integrated out and its vacuum expectation value induces mass of a charged fermion, whose sign is determined by $\operatorname{sgn}\left(\frac{q_{I} \xi}{m}\right)$. Thus, the effective IR theory is the Chern-Simons theory of level

$$
\kappa+\operatorname{sgn}\left(\frac{\xi}{m}\right) \sum_{I=1}^{N_{f}} \operatorname{sgn}\left(q_{I}\right) \frac{q_{I}^{2}}{2} .
$$

Its index contribution is the level multiplied by $\operatorname{sgn}(m)$, which is from the fermion partner of $P$ integrated out:

$$
\operatorname{sgn}(m) \kappa+\operatorname{sgn}(\xi) \sum_{I=1}^{N_{f}} \operatorname{sgn}\left(q_{I}\right) \frac{q_{I}^{2}}{2} .
$$

On the other hand, at $P=0$ with small $m_{I}$ assumed, we have a set of Higgs vacua around it; we have $\sum_{I \in \hat{I}_{+}} q_{I}^{2}$ of them for $\xi>0$ and $\sum_{I \in \hat{I}_{-}} q_{I}^{2}$ for $\xi<0$. Their index contribution is multiplied by -1 due to our sign convention. Combined with (4.102), the total index is given by

$$
\mathcal{I}^{3 d}=\operatorname{sgn}(m) \kappa-\sum_{I=1}^{N_{f}} \frac{q_{I}^{2}}{2},
$$

which is independent of $\xi$. 
For $|m| \ll \frac{N_{f}}{2}$, a vacuum sits either at $P=0$ or at $|P|=\frac{\xi}{\alpha}$. For $\frac{\xi}{\alpha}<0$, we have only Higgs vacua around $P=0$, which give the index

$$
\mathcal{I}^{3 d}=-\sum_{I \in \hat{I}_{ \pm}} q_{I}^{2}, \quad \pm \xi>0
$$

or equivalently

$$
\mathcal{I}^{3 d}=-\sum_{I \in \hat{I}_{ \pm}} q_{I}^{2}, \quad \mp \alpha>0
$$

since $\frac{\xi}{\alpha}<0$. For $\frac{\xi}{\alpha}>0$, on the other hand, we have extra topological vauca at $P= \pm \frac{\xi}{\alpha}$, described by Chern-Simons theories of level

$$
\kappa \pm \sum_{I=1}^{N_{f}} \operatorname{sgn}\left(q_{I}\right) \frac{q_{I}^{2}}{2}
$$

respectively. Their index contributions are multiplied by $\pm \operatorname{sgn}(\alpha)$ due to the massive fermion partner of $P$ :

$$
\operatorname{sgn}(\alpha)\left( \pm \kappa+\sum_{I=1}^{N_{f}} \operatorname{sgn}\left(q_{I}\right) \frac{q_{I}^{2}}{2}\right) .
$$

Combined with (4.104), the total index is again given by

$$
\mathcal{I}^{3 d}=-\sum_{I \in \hat{I}_{ \pm}} q_{I}^{2}, \quad \mp \alpha>0
$$

regardless of $\xi$. This, with (4.103), confirms the result (4.97). Our result also reproduces that of [28] for $N_{f}=1$, up to extra overall -1 .

Some dualities of $d=3 \mathcal{N}=1$ gauge theories. Now we explore dualities between $3 \mathrm{~d}$ $\mathcal{N}=1$ gauge theories with the computation technique of indices we have used throughout the paper. Before proceeding, let us remark that we focus on dualities each side of which is either a $\mathrm{SO}(2)$ gauge theory or a Wess-Zumino model [29]. Earlier discussion on $d=3$ $\mathcal{N}=1$ dualities has been made in [30-32] for a last few decades. For recent discoveries of wider variety of $d=3 \mathcal{N}=1$ dualities involving more complicated Wess-Zumino type interactions or non-Abelian gauge groups, we refer the readers to [25, 28, 33, 34].

We start from the following duality between an $\mathcal{N}=1$ QED and a Wess-Zumino model:

$$
\begin{array}{cc}
\mathrm{SO}(2)_{\frac{1}{2}} \text { with } 1 \text { flavor } \vec{Q} & \text { WZ model with } P, \vec{X} \\
W=\frac{m}{2}|\vec{Q}|^{2}-\frac{1}{4}|\vec{Q}|^{4} &
\end{array} \quad \begin{gathered}
W=P\left(|\vec{X}|^{2}+m\right)-\frac{1}{3} P^{3}
\end{gathered}
$$

where $m$ is the relevant deformation parameter. For the QED with the quartic superpotential, we have a general expression of the index in (4.57), which leads to the vanishing index for this example:

$$
\mathcal{I}_{\text {quartic } \mathrm{SO}(2)_{1 / 2}}^{3 d}=\kappa+\frac{1}{2}|q|^{2} \operatorname{sgn}\left(c_{W}\right)=\frac{1}{2}-\frac{1}{2}=0 .
$$


Although this result is independent of the relevant deformation parameter $m$, it is interpreted as the Witten index of the theory only when $m>0$ because we have the $S^{1}$ moduli space of vacua when $m<0$. On the right hand side, the index of the WZ can be obtained in various ways as explained in section 2 . We have

$$
\mathcal{I}_{\mathrm{WZ}}^{3 d}=0,
$$

which is also independent of $m$.

It was discussed in [29] that one can derive three different dualities involving Abelian gauge theories from the duality (4.109). The theories in (4.109) have a $\mathrm{SO}(2)$ global symmetry, which can be gauged with an extra Chern-Simons interaction. Turing on the Chern-Simons level $l=1,0,-1$, one has three different dualities as follows.

For $l=1$, we have the following $\mathcal{N}=1$ duality involving a pair of $d=3$ Abelian gauge theories, which have quartic and critical superpotentials ${ }^{5}$ respectively.

$$
\begin{aligned}
& \mathrm{SO}(2)_{-\frac{1}{2}} \text { with } 1 \text { flavor } \vec{Q} \longleftrightarrow \quad \mathrm{SO}(2)_{\frac{1}{2}} \text { with } 1 \text { flavor } \vec{X} \text { and a singlet } P \\
& W=\frac{m}{2}|\vec{Q}|^{2}-\frac{1}{4}|\vec{Q}|^{4} \quad \longleftrightarrow \quad W=P\left(|\vec{X}|^{2}+m\right)-\frac{1}{3} P^{3} .
\end{aligned}
$$

The semi-classical vacuum structure was drawn in [29] and it is consistent with the pathintegral computation below. For the quartic theory, we again use the formula (4.57), which reads

$$
\mathcal{I}_{\text {quartic } \mathrm{SO}(2)_{-1 / 2}}^{3 d}=\kappa+\frac{1}{2}|q|^{2} \operatorname{sgn}\left(c_{W}\right)=-\frac{1}{2}-\frac{1}{2}=-1 .
$$

For the critical theory on the right hand side, the index is given by

$$
\mathcal{I}^{3 d}=\kappa \cdot \mathcal{Z}_{\kappa}+\Omega_{0}
$$

where one can obtain each term from (4.71) and from (4.88) respectively. As a result, we have the index of the critical theory as follows:

$$
\mathcal{I}_{\text {critical } \mathrm{SO}(2)_{1 / 2}}^{3 d}=0 \cdot \kappa+\left(-\frac{1}{2}\right)-\frac{1}{2}=-1,
$$

which agrees with (4.113).

For $l=0$, we have a duality between a gapped theory of massive fields and an Abelian gauge theory:

$$
\begin{aligned}
& \text { Massive field } \vec{Q} \\
& W=\frac{m}{2}|\vec{Q}|^{2} \\
& \begin{array}{c}
\mathrm{SO}(2)_{-\frac{1}{2}} \text { with } 1 \text { flavor } \vec{X} \text { and a singlet } P \\
W=P\left(|\vec{X}|^{2}+m\right)+\frac{1}{2} P^{2} .
\end{array}
\end{aligned}
$$

Note that the superpotential on the right hand side is not the critical one; it has a quadratic interaction for $P$. Indeed, those theories have the $\mathcal{N}=2$ supersymmetry. For $m=0$, we

\footnotetext{
${ }^{5}$ This name came from [29] whose authors were inspired by $d=3$ bosonization, which maps the critical scalar theory (with $\phi^{4}$ quartic interaction) to regular fermions. Note that the superpotential of (r.h.s. ) in (4.112) induces quartic scalar interaction $|\vec{x}|^{4}$ to its bosonic potential.
} 
have a non-compact moduli space of vacua, which does not have a well-defined Witten index. Hence, we assume $m \neq 0$ for the computations below. The index of a single massive field is $\operatorname{sgn}(m)$. Assuming $m \neq 0$, the index of the left hand side is given by

$$
\mathcal{I}_{\text {massive }}^{3 d}=\operatorname{sgn}(m) \times \operatorname{sgn}(m)=1
$$

because we have two massive fields. On the right hand side, we have to take into account the one-loop correction to the superpotential. The one-loop corrected superpotential is given by

$$
W_{\text {one-loop }}=P\left(|\vec{X}|^{2}+m\right)+\frac{1}{2} P^{2}-\frac{1}{2} P|P| .
$$

Using the language of $W=P K(\rho)+L(\rho)+F(P)$, the index is determined by the following asymptotic values:

$$
\begin{gathered}
\lim _{\rho \rightarrow \infty} K(\rho)=\infty, \\
\lim _{P \rightarrow+\infty} F^{\prime}(P)=m, \\
\lim _{P \rightarrow-\infty} F^{\prime}(P)=-\infty
\end{gathered}
$$

with $\lim _{p \rightarrow p^{*}} F^{\prime}(p)$ negligible. In order to have an integer index, as before, we have to scale $m \rightarrow C m$ with $C \rightarrow \infty$. For $m>0$, we read the index from the formula (4.114):

$$
\mathcal{I}_{\text {quadratic }}^{3 d} \mathrm{SO}(2)_{1 / 2}=1 \cdot \kappa+0-\frac{1}{2}=-1 .
$$

For $m<0$, we have

$$
\mathcal{I}_{\text {quadratic } \mathrm{SO}(2)_{1 / 2}}^{3 d}=0 \cdot \kappa+\left(-\frac{1}{2}\right)-\frac{1}{2}=-1 .
$$

Thus, the index of the right hand side is -1 regardless of $m$. The Witten indices of the duality pair agree with each other up to sign. This sign discrepancy is explained by the fact that, in reaching the dual pairs, we have integrated out an $\mathrm{SO}(2)$ gauge multiplet on the left hand side of (4.116). The indices before and after this step differ by a sign due to the extra gaugino in this example and the one below. Alternatively, we can choose to keep this $\mathrm{SO}(2)$ and match the sign as well. Recall that something similar happens when we start with $\mathcal{N}=2$ Chern-Simons and choose to integrate out the $\Sigma$ part of the $\mathcal{N}=2$ vector multiplet.

The last duality with $l=-1$ is the following:

$$
\begin{array}{ccc}
\mathrm{SO}(2)_{\frac{3}{2}} \text { with } 1 \text { flavor } \vec{Q} & \longleftrightarrow & \begin{array}{c}
\mathrm{SO}(2)_{-\frac{3}{2}} \text { with } 1 \text { flavor } \vec{X} \text { and a singlet } P \\
W=\frac{m}{2}|\vec{Q}|^{2}-\frac{1}{4}|\vec{Q}|^{4}
\end{array} \\
W=P\left(|\vec{X}|^{2}+m\right)-\frac{1}{3} P^{3} .
\end{array}
$$

For the quartic theory on the left hand side, we have

$$
\mathcal{I}_{\text {quartic } \mathrm{SO}(2)_{3 / 2}}^{3 d}=\kappa+\frac{1}{2}|q|^{2} \operatorname{sgn}\left(c_{W}\right)=\frac{3}{2}-\frac{1}{2}=1 .
$$


The index of of the critical theory on the right hand side reads

$$
\mathcal{I}_{\text {critical } \mathrm{SO}(2)_{-3 / 2}}^{3 d}=0 \cdot \kappa+\left(-\frac{1}{2}\right)-\frac{1}{2}=-1 .
$$

The Witten indices agree with each other, again up to sign which is due to extra $\mathrm{SO}(2)_{-1}$ integrated out.

\section{Acknowledgments}

We would like to thank Vladimir Bashmakov for useful discussions. CH is grateful to KIAS for the kind hospitality where part of this work was done. $\mathrm{CH}$ is partially supported by the ERC-STG grant 637844-HBQFTNCER and by the INFN. This work is also supported in part by KIAS Individual Grants (PG071301 for DG and PG005704 for PY) at Korea Institute for Advanced Study.

\section{A Heat kernel for a Landau problem}

Let us compute the analog of

$$
\left\langle X^{\prime}\left|e^{s \partial^{2} / 2}\right| X\right\rangle=\frac{1}{2 \pi s} e^{-\left(X-X^{\prime}\right)^{2} / 2 s}
$$

for $\mathbb{R}^{2}$, when a uniform magnetic field is turned on, i.e., for

$$
-\frac{1}{2} \partial^{2} \quad \rightarrow \quad H_{B} \equiv \frac{1}{2}\left(\left(i \partial_{x}-y B / 2\right)^{2}+\left(i \partial_{y}+x B / 2\right)^{2}\right)
$$

with $B$ positive. The Hamiltonian can be rewritten as usual as

$$
H_{B}=B\left(a^{\dagger} a+\frac{1}{2}\right), \quad\left[a, a^{\dagger}\right]=1,
$$

via a Harmonic oscillator,

$$
a \equiv i \sqrt{\frac{2}{B}}\left(\partial_{\bar{z}}+B z / 4\right), \quad a^{\dagger} \equiv i \sqrt{\frac{2}{B}}\left(\partial_{z}-B \bar{z} / 4\right)
$$

where $z=x+i y$ and $\partial_{z}=\left(\partial_{x}-i \partial_{y}\right) / 2$ etc. The lowest Landau level wavefunctions at $H_{B}=B / 2$ are

$$
\begin{aligned}
\Psi_{m}^{(0)}(x, y) & =\sqrt{\frac{(B / 2)^{m+1}}{\pi m !}} z^{m} e^{-B z \bar{z} / 4} \\
& =\sqrt{\frac{(B / 2)^{m+1}}{\pi m !}}\left(-\frac{4}{B} \partial_{\bar{z}}\right)^{m} e^{-B z \bar{z} / 4}
\end{aligned}
$$

labeled by the eigenvalues $m \geq 0$ of the conserved angular momentum,

$$
L=-i x \partial_{y}+i y \partial_{x}
$$

Higher Landau level wavefunctions are generated as

$$
\Psi_{m}^{(n)}(x, y)=\frac{1}{\sqrt{n !}}\left(a^{\dagger}\right)^{n} \Psi_{m}^{(0)}(x, y), \quad n \geq 0
$$

with energy eigenvalues $(n+1 / 2) B$. 
The heat kernel of $H_{B}$, necessary for the computation of the path integral, is

$$
\begin{aligned}
\left\langle W\left|e^{-s H_{B}}\right| Z\right\rangle & =\sum_{n=0}^{\infty} e^{-s(n+1 / 2) B} \Delta^{(n)}(W, Z) \\
\Delta^{(n)}(W, Z) & \equiv \sum_{m=0}^{\infty} \Psi_{m}^{(n)}(W)^{*} \Psi_{m}^{(n)}(Z)
\end{aligned}
$$

The first contribution from $n=0$ is easy enough,

$$
\Delta^{(0)}(W, Z)=\frac{B}{2 \pi} e^{8 \partial_{\bar{z}} \partial_{w} / B} e^{-B(z \bar{z}+w \bar{w}) / 4}=\frac{B}{2 \pi} e^{-B(z \bar{z}+w \bar{w}-2 z \bar{w}) / 4} .
$$

For higher levels $n \geq 1$, we find

$$
\Delta^{(n)}(W, Z)=\frac{1}{n !}\left(\frac{2}{B}\right)^{n}\left(\partial_{z}-B \bar{z} / 4\right)^{n}\left(\partial_{\bar{w}}-B w / 4\right)^{n} \Delta^{(0)}(W, Z)
$$

which brings us to

$$
\left\langle W\left|e^{-s H_{B}}\right| Z\right\rangle=\left[\sum_{k=0}^{\infty} \frac{1}{(k !)^{2}} \cdot\left(\frac{e^{-s B}}{e^{-s B}-1} \cdot \frac{B|z-w|^{2}}{2}\right)^{k}\right] \cdot \frac{\Delta^{(0)}(W, Z)}{2 \sinh (s B / 2)} .
$$

The sum in the square bracket can be traded off in favor of a single integral. It reduces to, in the small $s$ limit which is relevant for the heat kernel expansion,

$$
\sim \frac{1}{2 \pi} \int_{0}^{2 \pi} d \theta e^{2 i \cos \theta \sqrt{|z-w|^{2} / 2 s}}
$$

playing the role of $\sim e^{-\left|X-X^{\prime}\right|^{2} / 2 s}$ of flat $\mathbb{R}^{2}$. The other factor similarly replaces $1 / 2 \pi s$.

These expressions say that, again, the heat kernel expansion becomes an expansion in positive powers of $s$, and the coincident limit is all that matters for the index-like computation. In the coincident limit $Z=W$, on the other hand, the heat kernel collapses to

$$
\left\langle Z\left|e^{-s H_{B}}\right| Z\right\rangle=\frac{B}{2 \pi} \cdot \frac{1}{2 \sinh (s B / 2)},
$$

which in the small $s$ limit again reduces to

$$
\simeq \frac{1}{2 \pi s}
$$

as is necessary for the usual heat kernel expansion for $\Omega$ to be applicable.

\section{B Pfaffian vs. Jacobian}

In this appendix, we derive the cancelation between the Pfaffian and the Jacobian given in (4.20). Note that, as a matter of notational ease, we now use $\vec{x}_{I}=\left(x_{I(1)}, x_{I(2)}\right) \rightarrow\left(x_{I}, x_{I}^{\prime}\right)$ and similarly $\vec{y}_{I}=\left(y_{I}, y_{I}^{\prime}\right)$ for Gaussian integral variables. 
First, let us consider the case of no neutral field. We want to show

$$
\operatorname{Pf}\left(\tilde{M}_{\kappa}\right)=-\operatorname{Det}\left(\frac{\partial\left(y_{I}, y_{I}^{\prime}, \mathbf{v}\right)}{\partial\left(x_{I}, x_{I}^{\prime}, \mathbf{u}\right)}\right) .
$$

for a radial superpotential. Let us examine the Pfaffian of fermion bilinear $\operatorname{Pf}\left(\tilde{M}_{\kappa}\right)$ on the left hand side of (B.1). We first note that $\tilde{M}_{\kappa}$ is written in the following block form:

$$
\tilde{M}_{\kappa}=\left(\begin{array}{cc}
L & Q \\
-Q^{T} & N
\end{array}\right)
$$

Each block is further decomposed into $2 \times 2$ blocks, each of which is labelled by doublet labels $I, J \in\left\{1, \cdots, N_{f}\right\} ;{ }^{6}$ For example, the top-left block $L$ is written as $L=\left(L_{I J}\right)$ where $L_{I J}$ is a $2 \times 2$ matrix defined by

$$
L_{I J}=\delta_{I J}\left(\begin{array}{cc}
0 & q_{I}\left(u_{x}+i u_{y}\right) \\
-q_{I}\left(u_{x}+i u_{y}\right) & 0
\end{array}\right) .
$$

In the same manner, $Q=\left(Q_{I J}\right)$ and $N=\left(N_{I J}\right)$ are also given by

$$
\begin{aligned}
& Q_{I J}=\left(\begin{array}{cc}
\partial_{I} \partial_{J} W & -\delta_{I J} q_{I} u_{z}+\partial_{I} \partial_{J}^{\prime} W \\
\delta_{I J} q_{I} u_{z}+\partial_{I}^{\prime} \partial_{J} W & \partial_{I}^{\prime} \partial_{J}^{\prime} W
\end{array}\right), \\
& N_{I J}=\delta_{I J}\left(\begin{array}{cc}
0 & -q_{I}\left(u_{x}-i u_{y}\right) \\
q_{I}\left(u_{x}-i u_{y}\right) & 0
\end{array}\right) .
\end{aligned}
$$

where $\partial_{I} \equiv \frac{\partial}{\partial x_{I}}$ and $\partial_{I}^{\prime} \equiv \frac{\partial}{\partial x_{I}^{\prime}}$.

Since $L$ is an $\left(2 N_{f}\right) \times\left(2 N_{f}\right)$ invertible matrix for generic $\mathbf{u}$, its inverse also reads in a block-diagonal form $L^{-1}=\left(\left(L^{-1}\right)_{I J}\right)$,

$$
\left(L^{-1}\right)_{I J}=\delta_{I J}\left(\begin{array}{cc}
0 & -\frac{1}{q_{I}\left(u_{x}+i u_{y}\right)} \\
\frac{1}{q_{I}\left(u_{x}+i u_{y}\right)} & 0
\end{array}\right) .
$$

Now the Pfaffian of bilinear $\operatorname{Pf}\left(\tilde{M}_{\kappa}\right)$ can be decomposed

$$
\operatorname{Pf}\left(\tilde{M}_{\kappa}\right)=\operatorname{Pf}(L) \operatorname{Pf}\left(N+Q^{T} L^{-1} Q\right) .
$$

\footnotetext{
${ }^{6}$ Throughout this appendix, we choose as a matter of computational convenience

$$
[\mathcal{D} \psi \mathcal{D} \lambda]^{(\text {appendix })} \equiv\left(d \lambda^{a} d \lambda_{a}\right)\left(\prod_{I=1}^{N_{f}} d \psi_{I(1)}^{b} d \psi_{I(2)}^{c}\right)\left(\prod_{J=1}^{N_{f}} d \psi_{J(1), b} d \psi_{J(2), c}\right)
$$

where super- and subscript $a, b$ and $c$ 's are spinor indices. This differs from our convention in the main text,

$$
[\mathcal{D} \psi \mathcal{D} \lambda]^{(\text {main text })} \equiv-\left(d \lambda^{a} d \lambda_{a}\right)\left(\prod_{I=1}^{N_{f}} d \psi_{I(1)}^{b} d \psi_{I(1), b} d \psi_{I(2)}^{c} d \psi_{I(2), c}\right)
$$
}

which translates to a relative sign factor $(-1)^{N_{f}+1}$. 
An auxiliary matrix $\hat{N}$ is defined

$$
\hat{N}=\left(u_{x}+i u_{y}\right)\left(N+Q^{T} L^{-1} Q\right),
$$

which is also in a block form $\hat{N}=\left(\hat{N}_{I J}\right)$, whose component for "radial" superpotential is given by

$$
\hat{N}_{I J}=\left(\begin{array}{cc}
\left(\frac{1}{q_{J}} x_{I} x_{J}^{\prime} W_{J}-\frac{1}{q_{I}} x_{I}^{\prime} x_{J} W_{I}\right) W_{I J} & -\delta_{I J} \frac{1}{q_{I}}\left(q_{I}^{2} \mathbf{u}^{2}+W_{I}^{2}\right) \\
\delta_{I J} \frac{1}{q_{I}}\left(q_{I}^{2} \mathbf{u}^{2}+W_{I}^{2}\right) & -\left(\frac{1}{q_{J}} x_{I} x_{J} W_{J}+\frac{1}{q_{I}} x_{I}^{\prime} x_{J}^{\prime} W_{I}\right) W_{I J} \\
+\left(\frac{1}{q_{J}} x_{I}^{\prime} x_{J}^{\prime} W_{J}+\frac{1}{q_{I}} x_{I} x_{J} W_{I}\right) W_{I J} & \left(\frac{1}{q_{I}} x_{I} x_{J}^{\prime} W_{I}-\frac{1}{q_{J}} x_{I}^{\prime} x_{J} W_{J}\right) W_{I J}
\end{array}\right) .
$$

In (B.9) we used the abbreviation for derivatives of superpotential: $W_{I}=\frac{\partial W}{\partial \rho_{I}}$ and $W_{I J}=\frac{\partial^{2} W}{\partial \rho_{I} \partial \rho_{J}}$.

According to (B.7) and the fact that the Pfaffian of $L$ is simply $\left(\prod_{I=1}^{N_{f}} q_{I}\left(u_{x}+i u_{y}\right)\right)$, the Pfaffian of $\tilde{M}_{\kappa}$ is given by

$$
\operatorname{Pf}\left(\tilde{M}_{\kappa}\right)=\left(\prod_{I=1}^{N_{f}} q_{I}\right) \operatorname{Pf}(\hat{N}) .
$$

Next let us move on to the Jacobian Det $\left(\frac{\partial\left(y_{I}, y_{I}^{\prime}, \mathbf{v}\right)}{\partial\left(x_{I}, x_{I}^{\prime}, \mathbf{u}\right)}\right)$ in the right hand side of (4.20). Recall that the new variables $\left\{y_{I}, y_{I}^{\prime}, \mathbf{v}\right\}$ are defined in terms of the original variables as follows:

$$
\begin{aligned}
y_{I} & =x_{I} \sqrt{q_{I}^{2} \mathbf{u}^{2}+W_{I}^{2}}, \\
y_{I}^{\prime} & =x_{I}^{\prime} \sqrt{q_{I}^{2} \mathbf{u}^{2}+W_{I}^{2}}, \\
\mathbf{v} & =\mathbf{u} .
\end{aligned}
$$

Its Jacobian matrix is written in a block form,

$$
J=\frac{\partial\left(y_{I}, y_{I}^{\prime}, \mathbf{v}\right)}{\partial\left(x_{I}, x_{I}^{\prime}, \mathbf{u}\right)}=\left(J_{I J}\right)
$$

where each $J_{I J}$ is a $2 \times 2$ matrix,

$$
J_{I J}=\left(\begin{array}{cc}
\delta_{I J} \sqrt{q_{I}^{2} \mathbf{u}^{2}+W_{I}^{2}}+\frac{x_{I} x_{J} W_{I} W_{I J}}{\sqrt{q_{I}^{2} \mathbf{u}^{2}+W_{I}^{2}}} & \frac{x_{I} x_{J}^{\prime} W_{I} W_{I J}}{\sqrt{q_{I}^{2} \mathbf{u}^{2}+W_{I}^{2}}} \\
\frac{x_{I}^{\prime} x_{J} W_{I} W_{I J}}{\sqrt{q_{I}^{2} \mathbf{u}^{2}+W_{I}^{2}}} & \delta_{I J} \sqrt{q_{I}^{2} \mathbf{u}^{2}+W_{I}^{2}}+\frac{x_{I}^{\prime} x_{J}^{\prime} W_{I} W_{I J}}{\sqrt{q_{I}^{2} \mathbf{u}^{2}+W_{I}^{2}}}
\end{array}\right) .
$$

Another auxiliary matrix $\hat{J}$ is defined by

$$
\hat{J}=\hat{N} J^{-1} .
$$


Surprisingly, $\hat{J}$ is given by $\hat{J}=\left(\hat{J}_{I J}\right)$ with

$$
\hat{J}_{I J}=\frac{1}{q_{J}}\left(\begin{array}{cc}
\frac{x_{I} x_{J}^{\prime} W_{J} W_{I J}}{\sqrt{q_{J}^{2} \mathbf{u}^{2}+W_{J}^{2}}} & -\delta_{I J} \sqrt{q_{J}^{2} \mathbf{u}^{2}+W_{J}^{2}}-\frac{x_{I} x_{J} W_{J} W_{I J}}{\sqrt{q_{J}^{2} \mathbf{u}^{2}+W_{J}^{2}}} \\
\delta_{I J} \sqrt{q_{J}^{2} \mathbf{u}^{2}+W_{J}^{2}}+\frac{x_{I}^{\prime} x_{J}^{\prime} W_{J} W_{I J}}{\sqrt{q_{J}^{2} \mathbf{u}^{2}+W_{J}^{2}}} & -\frac{x_{I}^{\prime} x_{J} W_{J} W_{I J}}{\sqrt{q_{J}^{2} \mathbf{u}^{2}+W_{J}^{2}}}
\end{array}\right) .
$$

Furthermore, one can figure out how to relate $\hat{J}$ and $J$,

$$
\hat{J}=J^{T} S
$$

where $S=\left(S_{I J}\right)$ is a block matrix whose component is

$$
S_{I J}=\frac{\delta_{I J}}{q_{J}}\left(\begin{array}{cc}
0 & -1 \\
1 & 0
\end{array}\right) .
$$

Hence, the determinant of $\hat{J}$ is given by

$$
\operatorname{Det}(\hat{J})=\operatorname{Det}(J) \operatorname{Det}(S)=\frac{\operatorname{Det}(J)}{\prod_{I=1}^{N_{f}} q_{I}^{2}},
$$

Using this relation, one can also obtain the determinant of $\hat{N}$ :

$$
\operatorname{Det}(\hat{N})=\operatorname{Det}(\hat{J}) \operatorname{Det}(J)=\left(\frac{\operatorname{Det}(J)}{\prod_{I=1}^{N_{f}} q_{I}}\right)^{2}
$$

and moreover the Pfaffian:

$$
\operatorname{Pf}(\hat{N})= \pm \frac{\operatorname{Det}(J)}{\prod_{I=1}^{N_{f}} q_{I}},
$$

where the sign is fixed by the recursive definition of Pfaffian. By combining (B.10) and (B.20)

$$
\operatorname{Pf}\left(\tilde{M}_{\kappa}\right)=(-1)^{N_{f}} \operatorname{Det}(J)
$$

Taking into account the convention of Pfaffian, we complete the proof of (4.20)

$$
\operatorname{Pf}\left(\tilde{M}_{\kappa}\right)^{(\text {main text })}=(-1)^{N_{f}+1} \operatorname{Pf}\left(\tilde{M}_{\kappa}\right)^{(\text {appendix })}=-\operatorname{Det}(J) .
$$

Now let us move on to the case with a single neutral field $P$. In that case, the Pfaffian matrix becomes

$$
\tilde{M}_{\kappa}=\left(\begin{array}{cc|c}
L & Q & R \\
-Q^{T} & N & S \\
\hline-R^{T} & -S^{T} & O
\end{array}\right)
$$


where $R=\left(R_{I}\right)$ and $S=\left(S_{I}\right)$ are $2 N_{f} \times 2$ matrices defined by

$$
\begin{aligned}
& R_{I}=\left(\begin{array}{ll}
0 & \partial_{I} W_{p} \\
0 & \partial_{I}^{\prime} W_{p}
\end{array}\right), \\
& S_{I}=\left(\begin{array}{ll}
-\partial_{I} W_{p} & 0 \\
-\partial_{I}^{\prime} W_{p} & 0
\end{array}\right)
\end{aligned}
$$

with $I=1, \ldots, N_{f}$; and $O$ is a $2 \times 2$ matrix:

$$
O=\left(\begin{array}{cc}
0 & W_{p p} \\
-W_{p p} & 0
\end{array}\right)
$$

Also $W_{p}$ and $W_{p p}$ are defined by $W_{p}=\frac{\partial W}{\partial p}$ and $W_{p p}=\frac{\partial^{2} W}{\partial p^{2}}$. Note that we have chosen a basis of the matrix such that the terms involving the neutral field are placed at the last part of the columns and rows.

Assuming non-zero $W_{p p}$, which can be taken to be zero in the end, one can apply the identity (B.7), with some exchanges of columns and rows understood, to this new $\tilde{M}_{\kappa}$. The Pfaffian of $\tilde{M}_{\kappa}$ is then decomposed into

$$
\operatorname{Pf}\left(\tilde{M}_{\kappa}\right)=W_{p p} \times \operatorname{Pf}\left(\begin{array}{cc}
L & Q-\hat{O} \\
-Q^{T}+\hat{O}^{T} & N
\end{array}\right) .
$$

where $\hat{O}=\left(\hat{O}_{I J}\right)$ is given by

$$
\hat{O}_{I J}=-R_{I} O_{I J}^{-1} S_{J}^{T}=\frac{1}{W_{p p}}\left(\begin{array}{cc}
\partial_{I} W_{p} \partial_{J} W_{p} & \partial_{I} W_{p} \partial_{J}^{\prime} W_{p} \\
\partial_{I}^{\prime} W_{p} \partial_{J} W_{p} & \partial_{I}^{\prime} W_{p} \partial_{J}^{\prime} W_{p}
\end{array}\right) .
$$

One can see that the presence of neutral field $P$ affects the above derivation for $\operatorname{Pf}\left(\tilde{M}_{\kappa}\right)$ without the neutral field in such a way that $Q$ is just replaced by $Q-\hat{O}$; or equivalently

$$
W_{I J} \quad \rightarrow \quad W_{I J}-\frac{W_{p I} W_{p J}}{W_{p p}} .
$$

Next, the Jacobian matrix with the neutral field $P$ is given by

$$
J=\left(\begin{array}{c|c}
J^{(0)} & G \\
\hline H & W_{p p}
\end{array}\right)
$$

where $J^{(0)}$ is the Jacobian matrix without the neutral field; $G=\left(G_{I}\right)$ is a $2 N_{f} \times 1$ matrix:

$$
G_{I}=\left(\begin{array}{c}
\frac{x_{I} W_{I} W_{p I}}{\sqrt{q_{I}^{2} \mathbf{u}^{2}+W_{I}^{2}}} \\
\frac{x_{I}^{\prime} W_{I} W_{p I}}{\sqrt{q_{I}^{2} \mathbf{u}^{2}+W_{I}^{2}}}
\end{array}\right)
$$

and $H=\left(H_{J}\right)$ is a $1 \times 2 N_{f}$ matrix:

$$
H_{J}=\left(x_{J} W_{p J} x_{J}^{\prime} W_{p J}\right)
$$


with $I, J=1, \ldots, N_{f}$. Using the identity

$$
\operatorname{Det}\left(\begin{array}{ll}
A & B \\
C & D
\end{array}\right)=\operatorname{Det}(D) \operatorname{Det}\left(A-B D^{-1} C\right),
$$

we obtains the Jacobian as follows:

$$
\operatorname{Det}(J)=W_{p p} \times \operatorname{Det}\left(J^{(0)}-\hat{K}\right)
$$

where $\hat{K}=\left(\hat{K}_{I J}\right)$ is given by

$$
\hat{K}_{I J}=\left(\begin{array}{cc}
\frac{x_{I} x_{J} W_{I} W_{p I} W_{p J}}{W_{p p} \sqrt{q_{I}^{2} \mathbf{u}^{2}+W_{I}^{2}}} & \frac{x_{I} x_{J}^{\prime} W_{I} W_{p I} W_{p J}}{W_{p p} \sqrt{q_{I}^{2} \mathbf{u}^{2}+W_{I}^{2}}} \\
\frac{x_{I}^{\prime} x_{J} W_{I} W_{p I} W_{p J}}{W_{p p} \sqrt{q_{I}^{2} \mathbf{u}^{2}+W_{I}^{2}}} & \frac{x_{I}^{\prime} x_{J}^{\prime} W_{I} W_{p I} W_{p J}}{W_{p p} \sqrt{q_{I}^{2} \mathbf{u}^{2}+W_{I}^{2}}}
\end{array}\right) .
$$

Recall $J^{(0)}$, which is given by (B.13). One can see that again the presence of the neutral field replaces $W_{I J}$ by $W_{I J}-\frac{W_{p I} W_{p J}}{W_{p p}}$ in the derivation of the Jacobian without the neutral field. Therefore, the proof of (B.22) above still holds with this replacement understood. With more than one neutral fields, one can perform the same procedure, which reduces the columns and rows involving neutral fields one by one.

Open Access. This article is distributed under the terms of the Creative Commons Attribution License (CC-BY 4.0), which permits any use, distribution and reproduction in any medium, provided the original author(s) and source are credited.

\section{References}

[1] M.F. Atiyah and I.M. Singer, The index of elliptic operators on compact manifolds, Bull. Am. Math. Soc. 69 (1969) 422 [INSPIRE].

[2] E. Witten, Constraints on supersymmetry breaking, Nucl. Phys. B 202 (1982) 253 [INSPIRE].

[3] L. Álvarez-Gaumé, Supersymmetry and the Atiyah-Singer index theorem, Commun. Math. Phys. 90 (1983) 161 [INSPIRE].

[4] F. Benini, R. Eager, K. Hori and Y. Tachikawa, Elliptic genera of two-dimensional $N=2$ gauge theories with rank-one gauge groups, Lett. Math. Phys. 104 (2014) 465 [arXiv: 1305.0533] [INSPIRE].

[5] F. Benini, R. Eager, K. Hori and Y. Tachikawa, Elliptic genera of $2 d \mathcal{N}=2$ gauge theories, Commun. Math. Phys. 333 (2015) 1241 [arXiv:1308.4896] [INSPIRE].

[6] K. Hori, H. Kim and P. Yi, Witten index and wall crossing, JHEP 01 (2015) 124 [arXiv: 1407.2567] [INSPIRE].

[7] G.W. Moore, N. Nekrasov and S. Shatashvili, D particle bound states and generalized instantons, Commun. Math. Phys. 209 (2000) 77 [hep-th/9803265] [INSPIRE].

[8] V. Pestun, Localization of gauge theory on a four-sphere and supersymmetric Wilson loops, Commun. Math. Phys. 313 (2012) 71 [arXiv:0712.2824] [InSPIRE].

[9] N. Seiberg, Naturalness versus supersymmetric nonrenormalization theorems, Phys. Lett. B 318 (1993) 469 [hep-ph/9309335] [INSPIRE]. 
[10] M.F. Atiyah and R. Bott, A Lefschetz fixed point formula for elliptic complexes. I, Annals Math. 86 (1967) 374.

[11] E. Witten, Phases of $N=2$ theories in two-dimensions, Nucl. Phys. B 403 (1993) 159 [hep-th/9301042] [INSPIRE].

[12] E. Witten, Supersymmetry and Morse theory, J. Diff. Geom. 17 (1982) 661.

[13] C. Hwang, J. Kim, S. Kim and J. Park, General instanton counting and 5d SCFT, JHEP 07 (2015) 063 [arXiv: 1406.6793] [INSPIRE].

[14] C. Cordova and S.-H. Shao, An index formula for supersymmetric quantum mechanics, arXiv: 1406.7853 [INSPIRE].

[15] S.J. Gates, M.T. Grisaru, M. Roček and W. Siegel, Superspace or one thousand and one lessons in supersymmetry, Front. Phys. 58 (1983) 1 [hep-th/0108200] [INSPIRE].

[16] R.A. Coles and G. Papadopoulos, The geometry of the one-dimensional supersymmetric nonlinear $\sigma$-models, Class. Quant. Grav. 7 (1990) 427 [INSPIRE].

[17] G.W. Gibbons, G. Papadopoulos and K.S. Stelle, HKT and OKT geometries on soliton black hole moduli spaces, Nucl. Phys. B 508 (1997) 623 [hep-th/9706207] [INSPIRE].

[18] H.P. McKean Jr. and I.M. Singer, Curvature and the eigenvalues of the Laplacian, J. Diff. Geom. 1 (1967) 43.

[19] S.-J. Lee and P. Yi, Witten index for noncompact dynamics, JHEP 06 (2016) 089 [arXiv: 1602.03530] [INSPIRE].

[20] C. Hwang and P. Yi, Twisted partition functions and H-saddles, JHEP 06 (2017) 045 [arXiv: 1704.08285] [INSPIRE].

[21] C. Hwang, S. Lee and P. Yi, Holonomy saddles and supersymmetry, Phys. Rev. D 97 (2018) 125013 [arXiv: 1801.05460] [INSPIRE].

[22] L. Di Pietro and Z. Komargodski, Cardy formulae for SUSY theories in $d=4$ and $d=6$, JHEP 12 (2014) 031 [arXiv: 1407.6061] [INSPIRE].

[23] O. Aharony et al., Aspects of $N=2$ supersymmetric gauge theories in three-dimensions, Nucl. Phys. B 499 (1997) 67 [hep-th/9703110] [InSPIRE].

[24] K. Intriligator and N. Seiberg, Aspects of $3 d N=2$ Chern-Simons-Matter Theories, JHEP 07 (2013) 079 [arXiv: 1305.1633] [inSPIRE].

[25] V. Bashmakov, J. Gomis, Z. Komargodski and A. Sharon, Phases of $\mathcal{N}=1$ theories in $2+1$ dimensions, JHEP 07 (2018) 123 [arXiv:1802.10130] [INSPIRE].

[26] C.-k. Lee, K.-M. Lee and E.J. Weinberg, Supersymmetry and selfdual Chern-Simons systems, Phys. Lett. B 243 (1990) 105 [INSPIRE].

[27] E. Witten, Supersymmetric index of three-dimensional gauge theory, hep-th/9903005 [INSPIRE].

[28] D. Gaiotto, Z. Komargodski and J. Wu, Curious aspects of three-dimensional $\mathcal{N}=1$ SCFTs, JHEP 08 (2018) 004 [arXiv: 1804.02018] [INSPIRE].

[29] F. Benini and S. Benvenuti, $\mathcal{N}=1$ dualities in $2+1$ dimensions, JHEP 11 (2018) 197 [arXiv: 1803.01784] [INSPIRE].

[30] M. Gremm and E. Katz, Mirror symmetry for $N=1$ QED in three-dimensions, JHEP 02 (2000) 008 [hep-th/9906020] [INSPIRE]. 
[31] S. Gukov and D. Tong, D-brane probes of special holonomy manifolds and dynamics of $N=1$ three-dimensional gauge theories, JHEP 04 (2002) 050 [hep-th/0202126] [INSPIRE].

[32] A. Armoni, A. Giveon, D. Israel and V. Niarchos, Brane dynamics and 3D Seiberg duality on the domain walls of $4 D N=1 S Y M$, JHEP 07 (2009) 061 [arXiv:0905.3195] [INSPIRE].

[33] F. Benini and S. Benvenuti, $N=1$ QED in $2+1$ dimensions: dualities and enhanced symmetries, arXiv: 1804.05707 [INSPIRE].

[34] C. Choi, M. Roček and A. Sharon, Dualities and phases of $3 D N=1 S Q C D$, JHEP 10 (2018) 105 [arXiv: 1808.02184] [INSPIRE]. 